\title{
QCD Sum Rules for Skeptics
}

\author{
DEREK B. LEINWEBER* \\ Department of Physics, Box 351560, University of Washington, Seattle, WA 98195 \\ and \\ TRIUMF, 4004 Wesbrook Mall, Vancouver, BC, V6T 2A3, Canada
}

(October 19, 1995)

(Revised July 10, 1996)

\begin{abstract}
A new Monte-Carlo based uncertainty analysis is introduced to quantitatively determine the predictive ability of QCD sum rules. A comprehensive analysis of ground state $\rho$-meson and nucleon spectral properties is performed. Many of the findings contradict the conventional wisdom of both practitioners and skeptics alike. Associations between the phenomenological fit parameters are particularly interesting as they reveal how the sum rules resolve the spectral properties. The use of derivative sum rules for the determination of $\rho$-meson spectral properties is shown to be a very unfavorable approach. Most prior nucleon sum rule analyses are based on a sum rule which is found to be invalid; the results are suspect, and should be reevaluated. The "Ioffe formula", argued by many to qualitatively encapsulate a description of the nucleon mass in terms of the chiral symmetry breaking order parameter $\langle\bar{q} q\rangle$ is misleading at best. QCD Sum Rules are found to be self-consistent without contributions from direct instantons. This implies that instanton effects are adequately accounted for in the nonperturbative vacuum condensates. This in-depth examination of QCD sum rule self consistency paints a favorable picture for further quantitative refinements of the QCD sum rule approach.
\end{abstract}

*E-mail: derek@phys.washington.edu • Telephone: (206) 616-1447 • Fax: (206) 685-0635 WWW: http://www.phys.washington.edu/ derek/Welcome.htm] 


\section{INTRODUCTION}

\section{A. Prologue}

The QCD Sum Rule (QCD-SR) approach to QCD continues to be a highly active field. The influence of the field is reflected in over 1500 references to the seminal paper of Shifman, Vainshtein and Zakharov (SVZ) [四], with over 380 of these references following the beginning of 1992 [2]. While the approach has been applied to a variety of hadronic observables in both vacuum and finite density nuclear matter, a comprehensive review of the systematic errors associated with the approach has not been considered.

One of the key assumptions of the approach is the use of the so called "continuum model" to remove excited state contaminations from the hadron correlator under investigation. Recently, the continuum model for the nucleon was tested by using correlation functions calculated via lattice regularized QCD [3:4]. The results suggest that the continuum model adequately removes the excited state contaminations and allows the isolation of the ground state. The success of the continuum model warrants a more careful investigation of other aspects of the QCD-SR approach so that uncertainties in the predictions may be reliably and quantitatively determined.

The focus of this paper is to establish a rigorous procedure for extracting quantities of phenomenological interest from QCD Sum Rules. The main conclusion of this investigation is that QCD Sum Rules work and they are predictive when the analysis is done rigorously. The ground state masses of the $\rho$-meson and the nucleon are used to introduce the concepts. However, the procedures introduced here are more generally applicable to quantities of current experimental interest. The goal is to shed light on the stability of QCD Sum Rule analyses and thus the accuracy to which observables may be reliably determined.

Implementation of the procedure introduced here does not lead to slight adjustments in the extracted parameters, but rather leads to qualitatively different conclusions. It will become apparent that most existing QCD-SR investigations of nucleon properties are in fact unreliable. Previous calculations are based on sum rules in which neither the operator product expansion (OPE) nor the phenomenological description are under acceptable control.

The QCD-SR method is presently the best fundamentally based approach for investigating the properties of hadrons in nuclear matter, as the lattice approach is challenged by a number of formidable obstacles [5]. Issues surrounding the finite width of the rho-meson make reliable lattice calculations of $\rho-\omega$ mixing very difficult. These are two of the many topics of current interest to nuclear physicists where the best fundamentally based approach is that of QCD-SRs. Moreover, the approach has minimal model dependence. Hence it is imperative to probe the predictive ability of the approach and the ideas presented here are currently being applied to these topics mentioned above [6] 8 .

\section{B. Systematic Uncertainties}

The most significant source of uncertainty in the QCD Sum Rule approach is an imprecise knowledge of the vacuum condensates appearing in the operator product expansion (OPE). 
This is particularly problematic for the higher dimension operators, where one usually invokes the vacuum saturation hypothesis (factorization) to replace higher-dimension-operator vacuum-expectation values by products of lower-dimension operators. A list of key sources of uncertainty in the QCD-SR approach should include:

- the unfactorized condensate values,

- factorization of higher dimensional operators,

- small but neglected $\alpha_{s}$ corrections,

- the truncation of the operator product expansion (OPE),

- the selection of the regime for matching the QCD and phenomenological sides of the sum rules,

- uncertainties associated with the summation of perturbation theory in large orders, and

- the possibility of significant direct instanton contributions.

With the exception of direct instanton contributions, the effects of these uncertainties will be estimated via a Monte-Carlo error analysis.

Gaussian distributions for the condensate values are generated via Monte Carlo. These distributions are selected to reflect the spread of values assumed in previously published QCD-SR analyses, and the uncertainties such as operator factorization listed above. These distributions provide a distribution for the OPE and thus uncertainty estimates for the OPE which will be used in a $\chi^{2}$ fit. In turn, the OPE distribution provides distributions for the phenomenological fit parameters, thus establishing the predictive ability of the QCD-SR approach.

\section{Conventional Analyses}

In the field of QCD inspired models, the focus is often on whether or not the model is able to encompass the known experimental data, as opposed to assessing the predictive potential of the method. A review of the QCD-SR literature leaves no doubt as to whether or not the QCD-SR method is able to encompass the established data. The poorly determined condensate values and arbitrary aspect of the matching Borel regime provides tremendous freedom in devising ways to achieve the desired result. In fact, it is difficult to find QCDSR-based predictions of hadronic observables which fail to agree with experiment. This is somewhat surprising, since the physics responsible for some observables may be poorly represented in a truncated OPE commonly limited to distances of less than $0.3 \mathrm{fm}$.

Most QCD-SR calculations of nucleon properties in the literature fall short in the analysis stage where the phenomenological parameters are determined by matching the QCD and phenomenological sides of the sum rules. Typical analysis short-comings include:

- selecting a single value for the Borel parameter which gives "nice" results. A Borel regime should be selected in order to evaluate the stability and reliability of the results. 
- a selection of the Borel regime without careful regard to OPE convergence円 nor maintaining ground state dominance of the phenomenological side of the sum rules.

- the fixing of search parameters (such as the continuum threshold) to preferred values. It will become apparent that this introduces a strong bias to the remaining fit parameters which may not reflect the properties of QCD.

- claiming an accuracy for QCD-SR predictions without supporting calculations. Occasionally a "stability analysis" [9,10] is considered, in which fit parameters are monitored as a single condensate value is varied. However, such analyses explore a relatively small corner of the condensate parameter space.

In general, former procedures adopted for matching the two sides of the sum rules lack the level of rigor which will be presented here.

This general lack of rigor can be traced back to the original paper of SVZ [1]. In their conclusions, they comment "we prefer not to deepen into the debris of computer calculations, sophisticated fit programs, arranging error bars here and there..." While this may be appropriate for a seminal paper on the QCD-SR approach, continuation of this philosophy has lead to strong negative criticism of the QCD-SR field. It is not uncommon to hear remarks such as "QCD Sum Rules are useful only if you know the answer." or "You can get anything you want from QCD Sum Rules." and "10\% here, 10\% there... Pretty soon you're talking about real numbers!" These remarks are unfortunate, as a rigorous and proficuous analysis is possible.

\section{Outline}

In this paper, a method for the quantitative determination of phenomenological quantities with uncertainties will be presented. Since this paper is directed to both QCD-Sum-Rule practitioners and skeptics alike, we begin by briefly outlining the QCD-SR approach in Section [1]. A more pedagogical review of the approach may be found in the recent review article of Ref. [11]. This section also introduces the "Ioffe formula" [12], argued by many to qualitatively encapsulate a description of the nucleon mass in terms of the chiral symmetry breaking order parameter $\langle\bar{q} q\rangle$.

Uncertainties associated with the evaluation of the OPE will be addressed via a new Monte-Carlo based procedure. Estimates for these uncertainties are presented in Section [III. Section IV details the Monte-Carlo uncertainty analysis and discusses the optimization algorithm and cautious convergence criteria used in the following.

Section $\square$ presents an analysis of $\rho$-meson sum rules. Here the criteria for the selection of the regime for matching the QCD and phenomenological sides of the sum rules is reviewed. Reasonable alternatives are considered via Monte Carlo.

\footnotetext{
${ }^{1}$ Here and in the following, "convergence" of the OPE simply means that the highest dimension terms considered in the OPE, with their Wilson coefficients calculated to leading order in perturbation theory, are small relative to the leading terms of the OPE.
} 
These considerations lead to the selection of the optimal interpolating field for nucleon sum rules. New insights into unconventional nucleon interpolators obtained from the lattice QCD investigation of Ref. [13] play a significant role here. Section V1 reviews the selection of the optimal nucleon interpolating field for correlators obtained from spin- $1 / 2$ interpolating fields. The effects of choosing non-optimal interpolators is demonstrated. Section VII explores additional sum rules obtained from the overlap of the generalized spin- $1 / 2$ and a spin-3/2 interpolator.

In Section VII] we explore the correlations between condensate values, fit parameters and sum rule consistency. These correlations serve to identify the roles of the leading terms

of the OPE in hadronic physics. The validity of the "Ioffe formula" is evaluated here, and these results may be of interest to those modeling the QCD vacuum. The contingency table analysis reveals that the intimate relationship between the quark condensate and the nucleon mass suggested in the "Ioffe formula" is invalid.

The necessity of direct instanton contributions to the QCD Sum Rules is determined in Section IX. We demonstrate that there is no evidence indicating that direct instanton contributions are required to maintain sum rule consistency.

The implications of the results of this analysis on coordinate space correlators are discussed in Section X. Finally, Section X] summarizes the findings of this in-depth investigation.

\section{QCD SUM RULE FORMALISM}

The underlying principle of field theoretic approaches to hadron phenomenology is the Ansatz of duality. That is, it is possible to simultaneously describe a hadron as quarks propagating in the QCD vacuum, and as a phenomenological field with the appropriate quantum numbers.

Hadron masses are extracted from an analysis of the two-point function

$$
\Pi(p)=i \int d^{4} x e^{i p \cdot x}\langle\Omega|T\{\chi(x) \bar{\chi}(0)\}| \Omega\rangle .
$$

The interpolating field $\chi$ is typically constructed from quark field operators combined to give the quantum numbers of the hadron under investigation. The $\rho$-meson interpolator related to the decay constant is

$$
\chi_{\mu}=\frac{1}{2}\left(\bar{u} \gamma_{\mu} u-\bar{d} \gamma_{\mu} d\right)
$$

To maintain maximal overlap with the ground state relative to excited states, only interpolators without derivatives are considered. For the nucleon we begin by considering the most general spin-1/2 nucleon interpolator

$$
\chi_{\mathcal{O}}=\chi_{1}+\beta \chi_{2},
$$

where

$$
\chi_{1}(x)=\epsilon^{a b c}\left(u^{T a}(x) C \gamma_{5} d^{b}(x)\right) u^{c}(x)
$$


and

$$
\chi_{2}(x)=\epsilon^{a b c}\left(u^{T a}(x) C d^{b}(x)\right) \gamma_{5} u^{c}(x) .
$$

$\chi_{1}(x)$ is the interpolator typically used in lattice QCD analyses. $\chi_{2}(x)$ vanishes in the nonrelativistic limit. However in a theory with light relativistic current quarks, there is no reason to exclude such an interpolating field a priori [14]. With the use of the Fierz relations, the combination of the above two interpolating fields for $\beta=-1$ may be written

$$
\begin{aligned}
\chi_{\mathrm{SR}}(x) & =\epsilon^{a b c}\left(u^{T a}(x) C \gamma_{\mu} u^{b}(x)\right) \gamma_{5} \gamma^{\mu} d^{c}(x), \\
& =2\left(\chi_{2}-\chi_{1}\right),
\end{aligned}
$$

giving the proton interpolating field advocated by Ioffe 15] and often found in QCD-SR calculations.

\section{A. Phenomenology}

At the phenomenological level one proceeds by inserting a complete set of eigenstates with the quantum numbers of the interpolator. The ability of the interpolator to annihilate a positive parity baryon to the QCD vacuum is described by the parameter $\lambda$ in

$$
\left\langle\Omega\left|\chi_{\mathcal{O}}(0)\right| i, p, s\right\rangle=\lambda_{\mathcal{O} i} u(p, s)
$$

for state $i$ of momentum $p$ and spin $s . u(p, s)$ is a Dirac spinor. Negative parity states require a $\gamma_{5}$ on the right-hand side of (2.6). For the nucleon, the two point function has the form

$$
\Pi(p)=\sum_{i} \lambda_{\mathcal{O} i}^{2} \frac{\gamma \cdot p \pm M_{i}}{p^{2}-M_{i}^{2}-i \epsilon^{\prime}}
$$

where $+/-$ corresponds to positive/negative parity baryons. While there is some suppression of excited state contributions to the two-point function, there is little hope of isolating ground state properties from such a function. Hence one uses the celebrated Borel Transform

$$
\widehat{B}\left[f\left(p^{2}\right)\right]=\lim _{\substack{-p^{2} \rightarrow \infty \\ n \rightarrow \infty \\-p^{2} / n=M^{2}}} \frac{1}{n !}\left(-p^{2}\right)^{n+1}\left(\frac{d}{d p^{2}}\right)^{n} f\left(p^{2}\right)
$$

at each Dirac- $\gamma$ structure to obtain exponential suppression of excited states. The parameter $M$ is commonly referred to as the Borel mass. The Borel transform is easily applied to a dispersion relation for $\Pi(p)$ at each Dirac- $\gamma$ structure

$$
\Pi\left(p^{2}\right)=\frac{1}{\pi} \int_{0}^{\infty} d s \frac{\operatorname{Im} \Pi(s)}{s-p^{2}}+\text { subtractions },
$$

and the polynomial subtraction terms are eliminated by the Borel transform. At the structure $\gamma \cdot p$ one has 


$$
\Pi_{\gamma \cdot p}(M)=\int \rho_{\gamma \cdot p}(s) e^{-s / M^{2}} d s,
$$

where we have introduced the spectral density $\rho(s)=\operatorname{Im} \Pi(s) / \pi$,

$$
\rho_{\gamma \cdot p}(s)=\lambda_{\mathcal{O}}^{2} \delta\left(s-M_{N}^{2}\right)+\xi_{\gamma \cdot p}(s) \text {. }
$$

At the structure 1

$$
\Pi_{1}(M)=\int \rho_{1}(s) e^{-s / M^{2}} d s
$$

where

$$
\rho_{1}(s)=\left( \pm M_{N}\right) \lambda_{\mathcal{O}}^{2} \delta\left(s-M_{N}^{2}\right)+\xi_{1}(s)
$$

Here, $\xi_{\gamma \cdot p}(s)$ and $\xi_{1}(s)$ account for excited state contributions, and $+/-$ in $2.11 \mathrm{~b}$ ) corresponds to positive/negative parity states.

For the vector meson one has

$$
\begin{gathered}
\Pi_{V}(M)=\int \rho_{V}(s) e^{-s / M^{2}} d s, \\
\rho_{V}(s)=f_{\rho}^{2} \delta\left(s-M_{\rho}^{2}\right)+\xi_{V}(s),
\end{gathered}
$$

at the structure $g_{\mu \nu}-p_{\mu} p_{\nu} / p^{2}$.

\section{B. QCD}

At the quark level, one exploits the operator product expansion (OPE) to describe the short distance behavior of the two-point function

$$
\begin{aligned}
T\{\chi(x), \bar{\chi}(0)\}= & \sum_{n} C_{n}(x, \mu) \mathcal{O}_{n}(0, \mu) \\
= & C_{0}(x) I \\
& +C_{1}(x) m_{q} \\
& +C_{3}(x) \bar{q} q \\
& +C_{4.1}(x) G_{\mu \nu}^{a} G^{a \mu \nu}+C_{4.2}(x) m_{q} \bar{q} q \\
& +C_{5.1}(x) \bar{q} \sigma_{\mu \nu} \frac{\lambda^{a}}{2} q G^{a \mu \nu}+C_{5.2}(x) m_{q} G_{\mu \nu}^{a} G^{a \mu \nu} \\
& +C_{6.1}(x) \bar{q} \Gamma q \bar{q} \Gamma q+C_{6.2}(x) m_{q} \bar{q} \sigma_{\mu \nu} \frac{\lambda^{a}}{2} q G^{a \mu \nu}+C_{6.3}(x) f_{a b c} G_{\mu \nu}^{a} G_{\nu \lambda}^{b} G_{\lambda \mu}^{c} \\
& +C_{7.1}(x) \bar{q} q G_{\mu \nu}^{a} G^{a \mu \nu}+C_{7.2}(x) m_{q} \bar{q} \Gamma q \bar{q} \Gamma q+C_{7.3}(x) m_{q} f_{a b c} G_{\mu \nu}^{a} G_{\nu \lambda}^{b} G_{\lambda \mu}^{c} \\
& +C_{8.1}(x) \bar{q} \Gamma q \bar{q} \Gamma \sigma_{\mu \nu} \frac{\lambda^{a}}{2} q G^{a \mu \nu}+C_{8.2}(x) m_{q} \bar{q} q G_{\mu \nu}^{a} G^{a \mu \nu} \\
& +C_{8.3}(x) G_{\mu \nu}^{a} G^{a \mu \nu} G_{\rho \lambda}^{b} G^{b \rho \lambda},
\end{aligned}
$$


where $\mu$ is the normalization point at which the coefficient functions and the operators are defined. Here we have explicitly included all operators up to dimension eight, to leading order in the quark mass $m_{q}$, having the quantum numbers of the vacuum. The first digit of the subscript of the Wilson coefficients $C(x)$ gives the energy dimension of the operator. $\Gamma$ may take any of the 16 independent Dirac- $\gamma$ matrices.

Upon Fourier transforming to momentum space, (2.13) becomes an expansion in $1 / Q^{2}$, valid for large momentum transfers. Thus one may use perturbation theory to calculate the Wilson coefficients.

The contribution of the gluon operator term 4.1 in the expansion for the proton is suppressed due a factor of $(2 \pi)^{2}$ which appears in the denominator of the Wilson coefficient arising from integration over the quark-loop momentum which introduces the factor $d^{4} q /(2 \pi)^{4}$. Terms involving more than two gluon field strength tensors $G_{\mu \nu}^{a}$ and products of $m_{q}$ and $G_{\mu \nu}^{a} G^{a \mu \nu}$ are estimated to be small and are typically neglected.

The standard treatment of the OPE proceeds via inserting the explicit forms of the interpolating fields into the two-point function of (2.1) and contracting out pairs of timeordered quark-field operators which are the fully interacting quark propagators of QCD. Wick's theorem for the time-ordered product of two fermion fields provides

$$
\begin{aligned}
\langle\Omega| T & \left\{q^{a}(x), \bar{q}^{b}(0)\right\}|\Omega\rangle \\
& =\left\langle 0\left|T\left\{q^{a}(x), \bar{q}^{b}(0)\right\}\right| 0\right\rangle+\left\langle\Omega\left|: q^{a}(x), \bar{q}^{b}(0):\right| \Omega\right\rangle .
\end{aligned}
$$

Taylor expansion of the normal ordered piece leads to

$$
\begin{aligned}
: q_{\alpha}^{a}(x), \bar{q}_{\beta}^{b}(0):= & : q_{\alpha}^{a}(0), \bar{q}_{\beta}^{b}(0):+x^{\mu}: \partial_{\mu} q_{\alpha}^{a}(0), \bar{q}_{\beta}^{b}(0): \\
& +\frac{1}{2 !} x^{\mu} x^{\nu}: \partial_{\mu} \partial_{\nu} q_{\alpha}^{a}(0), \bar{q}_{\beta}^{b}(0):+\cdots
\end{aligned}
$$

The well known trick is to select the coordinate gauge $x^{\mu} A_{\mu}=0$ such that

$$
A_{\mu}(x)=-\frac{1}{2} G_{\mu \nu}(0) x^{\nu}-\frac{1}{3}\left(\partial_{\lambda} G_{\mu \nu}(0)\right) x^{\lambda} x^{\nu}+\cdots
$$

and the partial derivatives of (2.15) may be replaced by covariant derivatives

$$
\partial_{\mu} \rightarrow \nabla_{\mu}=\partial_{\mu}+i g A_{\mu}
$$

Generally, one works with (2.16) to leading order. The following relations allow an easy determination of the covariant derivatives

$$
\begin{gathered}
\gamma^{\mu} \nabla_{\mu} q_{\alpha}^{a}=-i m_{q} q_{\alpha}^{a}, \\
{\left[\nabla^{\mu}, \nabla^{\nu}\right]=i g_{c} G^{\mu \nu},} \\
\nabla^{2} q_{\alpha}^{a}=-\frac{1}{2} g_{c} \sigma \cdot G q_{\alpha}^{a} .
\end{gathered}
$$


In summary, the quark correlators employed in calculating the OPE under the usual assumption of vacuum saturation of the intermediate states of composite operators include

$$
\begin{aligned}
\langle\Omega| T & \left\{q^{a}(x), \bar{q}^{b}(0)\right\}|\Omega\rangle= \\
& \frac{i}{2 \pi^{2} x^{4}} \gamma \cdot x \delta^{a b}-\frac{m_{q}}{2^{2} \pi^{2} x^{2}} \delta^{a b}-\frac{1}{2^{2} 3}\langle\bar{q} q\rangle \delta^{a b} \\
& +\frac{i}{2^{4} 3} m_{q}\langle\bar{q} q\rangle \gamma \cdot x \delta^{a b}+\frac{x^{2}}{2^{6} 3}\left\langle\bar{q} g_{c} \sigma \cdot G q\right\rangle \delta^{a b} \\
& -\frac{i x^{2}}{2^{7} 3^{2}} m_{q}\left\langle\bar{q} g_{c} \sigma \cdot G q\right\rangle \gamma \cdot x \delta^{a b}-\frac{x^{4}}{2^{10} 3^{3}}\langle\bar{q} q\rangle\left\langle g_{c}^{2} G^{2}\right\rangle \delta^{a b} \\
& +\frac{i}{2^{5} \pi^{2} x^{2}}\left(g_{c} G_{\alpha \beta}^{n}\right)\left(\gamma \cdot x \sigma^{\alpha \beta}+\sigma^{\alpha \beta} \gamma \cdot x\right) \frac{\lambda_{a b}^{n}}{2} \\
& +\frac{1}{2^{5} \pi^{2}} m_{q}\left[\ln \left(\frac{-x^{2} \Lambda^{2}}{4}\right)+2 \gamma_{E M}\right]\left(g_{c} G_{\alpha \beta}^{n}\right) \sigma^{\alpha \beta} \frac{\lambda_{a b}^{n}}{2} . \\
\langle\Omega| T & \left\{q^{a}(x) g_{c} G_{\alpha \beta}^{n} \bar{q}^{b}(0)\right\}|\Omega\rangle= \\
& -\frac{1}{2^{6} 3}\left\langle\bar{q} g_{c} \sigma \cdot G q\right\rangle \sigma_{\alpha \beta} \frac{\lambda_{a b}^{n}}{2} \\
& +\frac{i}{2^{8} 3} m_{q}\left\langle\bar{q} g_{c} \sigma \cdot G q\right\rangle\left(\gamma \cdot x \sigma_{\alpha \beta}+\sigma_{\alpha \beta} \gamma \cdot x\right) \frac{\lambda_{a b}^{n}}{2} \\
& +\frac{x^{2}}{2^{10} 3^{2}}\left\langle g_{c}^{2} G^{2}\right\rangle\langle\bar{q} q\rangle \sigma_{\alpha \beta} \frac{\lambda_{a b}^{n}}{2} .
\end{aligned}
$$

Finally the momentum space correlator is Borel transformed. Here one encounters the factorial suppression of higher-dimension operators as indicated in

$$
\widehat{B}\left[\frac{1}{\left(p^{2}\right)^{k}}\right]=(-1)^{k} \frac{1}{(k-1) !} \frac{1}{\left(M^{2}\right)^{k-1}} .
$$

It is worth noting that the factorial suppression does not really set in until one reaches the term $1 / M^{6}$. For the $\rho$ meson this term corresponds to an operator of dimension eight, and for the nucleon the dimensions are twelve and thirteen for the two nucleon sum rules obtained from spin-1/2 interpolating fields. Since the OPE for the $\rho$-meson is traditionally truncated at dimension eight [1], the correlator should be more reliable than for the nucleon sum rules which are truncated at dimension nine.

\section{Power Corrections from the Large-Order Behavior of Perturbation Theory}

It is well known that the standard treatment of the OPE encounters difficulties at large orders in the perturbative expansion of the Wilson coefficients. Perturbative expansions are divergent at large orders and are asymptotic at best [16]. In the standard treatment, these divergences limit the accuracy to which the perturbative coefficients may be determined.

Renormalons [17], a particular set of perturbative graphs, provide a simple illustration of the factorial growth in the contributions of perturbative graphs at large orders [18 22]. The factorial growth in large-order contributions associated with soft infrared (IR) virtual 
momenta is problematic, as the series is not Borel summable. As such, the Wilson coefficients, by themselves, are ill defined in the standard treatment. The uncertainty resulting from the restriction to finite orders of the asymptotic expansion is commonly referred to as the IR renormalon ambiguity. Of course the use of perturbative propagators to describe soft virtual momenta is incorrect. Instead, one must utilize fully dressed nonperturbative propagators. Hence, it is not surprising one encounters difficulties in the IR regime, as one is simply encountering the Landau pole of the perturbative coupling constant.

A solution to the IR renormalon ambiguity problem was provided by the ITEP group [23] through the introduction of the normalization/separation scale $\mu$ of (2.13a). The OPE becomes a separation of scales, with virtual momenta lying above $\mu$ represented in the Wilson coefficients, and momenta below $\mu$ in the vacuum expectation values (VEVs) of higher-dimension operators beginning at dimension four. The introduction of the scale $\mu$ eliminates the IR renormalon ambiguity of the perturbative expansion. However, in principle, both the Wilson coefficients and the VEVs of operators contain perturbative as well as nonperturbative physics. [

In principle, the OPE defined in (2.13a) is free of IR renormalon ambiguities. However, in practice it is difficult to implement the formal procedure of truncating virtual momenta below $\mu$ when calculating higher order $\alpha_{s}$ corrections to the Wilson coefficients. While these contributions are small for leading order corrections, their inclusion is a source of error which can be accommodated in the Monte-Carlo uncertainty analysis.

In the ultraviolet (UV) virtual momenta regime, the renormalon series is sign alternating and Borel summable. Summation of the UV series can give rise to a power correction proportional to $\Lambda_{\mathrm{QCD}}^{2} / Q^{2}$ which closely resembles the squared quark mass contribution to the OPE [21]. For the vector correlator, the UV renormalon gives rise to an effective mass of roughly $(2 / \pi)^{1 / 2} \Lambda_{\mathrm{QCD}} / 3$, which is much larger than the usual current quark mass the order of $5 \mathrm{MeV}$ [21]. Hence there is some concern that important power corrections associated with UV renormalons has been overlooked in the standard QCD-SR treatment. Moreover, it is the power corrections that provide the link to nonperturbative phenomena. To the extent that the power correction is scheme dependent and not reliably known, we will refer to these quark-mass like power corrections as UV renormalon uncertainties.

Additional evidence for $1 / q^{2}$-type power corrections independent of renormalons has been obtained through a consideration of constraints imposed by asymptotic freedom and analyticity on the large-order behavior of perturbation theory [24,25]. In this case the perturbative series is not Borel summable. Given the absence of a local gauge-invariant fieldoperator product of dimension two, some might argue that the coefficient of the singularity in the Borel plane governing the large-order behavior of perturbative series must have a zero. However, perturbative estimates of the coefficient do not provide evidence of such a zero 25] and the issue is unsettled.

Hence the issue of possible dimension-two power corrections is of current interest. The techniques presented in this manuscript might be used to provide some phenomenological

\footnotetext{
${ }^{2}$ For a particularly lucid discussion of why the standard treatment of the OPE is phenomenologically successful, see Ref. [20].
} 
insight into the debate. However, such considerations would take us too far afield and will be deferred to a subsequent analysis [26]. On the other hand, it is interesting to look for discrepancies which might be rooted in such power corrections, and this is done in the following analysis.

\section{Ioffe Formula}

To summarize the points of this section, we present the traditional QCD Sum Rules for the nucleon [15] obtained from the consideration of the interpolating field $\chi_{\mathrm{SR}} / 2$ of (2.5) to operator dimension 8.

$$
\begin{aligned}
& \text { At } \gamma \cdot p: \quad \frac{1}{8} \frac{1}{(2 \pi)^{4}} M^{6}+\frac{1}{32} \frac{1}{(2 \pi)^{2}}\left\langle\frac{\alpha_{s}}{\pi} G^{2}\right\rangle M^{2}+\frac{1}{6}\langle\bar{q} q\rangle^{2} \\
& +\frac{1}{24}\langle\bar{q} g \sigma \cdot G q\rangle\langle\bar{q} q\rangle \frac{1}{M^{2}} \\
& =\lambda_{N}^{2} e^{-M_{N}^{2} / M^{2}}+\cdots \text {. } \\
& \text { At } 1: \quad-\frac{1}{4} \frac{1}{(2 \pi)^{2}}\langle\bar{q} q\rangle M^{4}+\frac{5}{288}\langle\bar{q} q\rangle\left\langle\frac{\alpha_{s}}{\pi} G^{2}\right\rangle \\
& =\lambda_{N}^{2} M_{N} e^{-M_{N}^{2} / M^{2}}+\cdots \text {. }
\end{aligned}
$$

The dots allow for excited state contributions.队

The Ioffe Formula for the nucleon mass is obtained from (2.22) by keeping the leading terms of the OPEs and assuming dominance of the ground state on the right-hand side of the equations,

$$
\begin{aligned}
\frac{1}{8} \frac{1}{(2 \pi)^{4}} M^{6} & \simeq \lambda_{N}^{2} e^{-M_{N}^{2} / M^{2}} \\
-\frac{1}{4} \frac{1}{(2 \pi)^{2}}\langle\bar{q} q\rangle M^{4} & \simeq \lambda_{N}^{2} M_{N} e^{-M_{N}^{2} / M^{2}} .
\end{aligned}
$$

The Ioffe formula follows from the ratio of these two equations,

$$
\begin{aligned}
M_{N} & \simeq-\frac{2(2 \pi)^{2}\langle\bar{q} q\rangle}{M^{2}} \\
& =0.90 \mathrm{GeV} \text { for } M=1 \mathrm{GeV} .
\end{aligned}
$$

If one prefers to eliminate the Borel mass by selecting $M=M_{N}$,

\footnotetext{
${ }^{3}$ The Wilson coefficient of the dimension 7 operator in $2.22 \mathrm{~b}$ ) has had an elusive history. Early calculations [10,27,28] appear to have calculated a subset of the diagrams contributing to the dimension 7 operator [9]. However a more persistent error lies in a factor of 3 correction in the term $\left[x^{2} /\left(2^{10} 3^{2}\right)\right]\langle\bar{q} q\rangle\left\langle g_{c}^{2} G^{2}\right\rangle \sigma_{\alpha \beta} \lambda_{a b}^{n} / 2$ of 2.20 from that used in previous analyses.
} 


$$
\begin{aligned}
M_{N} & \simeq\left(-2(2 \pi)^{2}\langle\bar{q} q\rangle\right)^{1 / 3} \\
& =0.97 \mathrm{GeV} .
\end{aligned}
$$

Here the quark condensate is estimated from the partially conserved axial current (PCAC) relation and pion decay to give

$$
\begin{aligned}
\langle\bar{q} q\rangle & =-\frac{m_{\pi}^{2} f_{\pi}^{2}}{m_{u}+m_{d}}, \\
& =(0.225 \mathrm{GeV})^{3},
\end{aligned}
$$

which corresponds to $m_{\pi}=.140 \mathrm{GeV}, f_{\pi}=0.093 \mathrm{GeV}$ and the average light quark mass is $7 \mathrm{MeV}$. The qualitative features of chiral symmetry breaking coupled with the quantitative success of these relations has lead to enormous faith being placed into the validity of the Ioffe formula. Most practitioners expect this relation to be valid, at least in a qualitative manner of proportionality.

\section{E. Excited State Contributions}

The operator product expansion provides knowledge of the two-point function (2.1) in the near perturbative regime. As such the correlator represents contributions from both the ground state hadron and excited states. To maintain some predictive ability in the QCD-SR approach, a "continuum model" for excited state contributions to the correlator is introduced.

Guided by the principle of duality, the leading terms of the OPE surviving in the limit $M \rightarrow \infty$ are used to model the form of the spectral density accounting for excited state contributions. Consider, as an example, the identity operator for the nucleon interpolator

$\chi_{\mathrm{SR}}$ of (2.5). Explicitly including the excited state term of (2.10) in equation (2.22a) leads to

$$
\frac{1}{2} \frac{1}{(2 \pi)^{4}} M^{6}=\int_{0}^{\infty} \xi_{\gamma \cdot p}(s) e^{-s / M^{2}} d s
$$

for large Borel mass. The Laplace transform readily provides

$$
\xi_{\gamma \cdot p}(s)=\frac{1}{2} \frac{1}{(2 \pi)^{4}} \frac{1}{2 !} s^{2} .
$$

The continuum model contribution is obtained by introducing a threshold, $s_{0}$, at which strength in the excited states becomes significant. Such a model is motivated well by the experimental cross section for $e^{+} e^{-} \rightarrow$ hadrons. In addition, this approach accommodates the finite widths and the multi-particle continuum of QCD. Hence the excited state contributions associated with the identity operator are

$$
\int_{s_{0}=w^{2}}^{\infty} \xi_{\gamma \cdot p}(s) e^{-s / M^{2}} d s=\frac{1}{2} \frac{1}{(2 \pi)^{4}} M^{6} e^{-w^{2} / M^{2}}\left(\frac{w^{4}}{2 M^{4}}+\frac{w^{2}}{M^{2}}+1\right) .
$$


Continuum model contributions associated with $M^{4}$ and $M^{2}$ terms are calculated in a similar manner. Usually the continuum contributions are placed on the OPE side of the sum rules. Hence, terms surviving in the limit $M \rightarrow \infty$ have the following factors associated with them:

$$
\begin{aligned}
& M^{6}:\left[1-e^{-w^{2} / M^{2}}\left(\frac{w^{4}}{2 M^{4}}+\frac{w^{2}}{M^{2}}+1\right)\right], \\
& M^{4}:\left[1-e^{-w^{2} / M^{2}}\left(\frac{w^{2}}{M^{2}}+1\right)\right], \\
& M^{2}:\left[1-e^{-w^{2} / M^{2}}\right] .
\end{aligned}
$$

The contributions of this model relative to the ground state, whose properties one is really trying to determine, are not small. They are typically 10 to $50 \%$ [9]. The validity of this somewhat crude model is relied upon to cleanly remove the excited state contaminations.

In the lattice QCD investigation of the continuum model formulated in Euclidean space [3], the short time regime of point-to-point lattice correlation functions is described well by the QCD-Sum-Rule-inspired continuum model. There, the Laplace transform of the spectral density appears to be sufficient to render any structure in the spectral density insignificant in the short Euclidean time regime of point-to-point correlators. Similar conclusions are expected to hold for the Borel transformed sum rules under investigation here.

It should be noted that the spectral density of the physical world is different than that encountered on the lattice. For example, in the nucleon channel one expects strength in the correlator above the ground state to start at the $\pi p$ threshold [29], whereas in the lattice calculations such contributions are altered by the heavier quark mass and the quenched approximation. While the lattice results are encouraging, one may find that in some cases the continuum model is not sufficiently detailed when the continuum model contributions are large. An example of this will be presented in the discussion surrounding the $\rho$-meson sum rules, where discrepancies may be due to an over simplified continuum model.

\section{UNCERTAINTY ESTIMATES}

The origin of the uncertainty estimate for the OPE lies in the uncertainties assigned to the QCD parameters appearing in the truncated OPE. In this section we assess the uncertainties on these QCD parameters.

In assigning these uncertainties one would like to select values conservatively enough such that the QCD-SR approach can test the validity of our present understanding of QCD. QCDSR predictions that fail to agree with experiment at the $2 \sigma$ level should indicate a possibly interesting discrepancy. At the same time one would also like to select uncertainties in accord with the generally accepted values in the literature. In addition, the error assessments need to reflect uncertainties associated with the factorization of higher dimensional operators and small but never-the-less neglected $\alpha_{s}$ corrections. These considerations have been balanced in the following. While some may argue that some values are known better than that indicated here, others will no doubt argue that the errors are underestimated.

In any event, one will learn for the first time how the uncertainties in the QCD parameters are mapped into uncertainties in the phenomenological fit parameters. In the lattice QCD 
investigation of Ref. [3] it was noted that interplay between the pole position and continuum threshold lead to rather large uncertainties in the fit parameters. It will be interesting to see how reasonable estimates of the QCD uncertainties are revealed in the fit parameters.

For the quark condensate, $\langle\bar{q} q\rangle$ we note that there are two values commonly found in QCD-SR analyses. PCAC and pion decay considerations coupled with quark masses of $m_{u}+m_{d}=11 \mathrm{MeV}$ lead to a value of $-(0.250)^{3} \mathrm{GeV}^{3}$. QCD-SR considerations of $g_{A}$ and octet baryon magnetic moments 30 32] prefer a smaller magnitude at $-(0.225)^{3} \mathrm{GeV}^{3}$. Expecting the value to lie some where in this regime, we take the average of these values with an uncertainty of half the difference at an $80 \%$ confidence level. Introducing the standard QCD-SR notation we define

$$
a=-(2 \pi)^{2}\langle\bar{q} q\rangle=0.52 \pm .05 \mathrm{GeV}^{3} .
$$

This value and uncertainty is also in accord with $a=0.47 \pm 0.05 \mathrm{GeV}^{3}$ obtained from more recent quark mass estimates [33].

Early estimates of the gluon condensate $\left\langle\alpha_{s} / \pi G^{2}\right\rangle$ from charmonium sum rules [四] place the value at $0.012 \pm 0.005 \mathrm{GeV}^{4}$, which is generally referred to as the "standard value"

$$
b=(2 \pi)^{2}\left\langle\frac{\alpha_{s}}{\pi} G^{2}\right\rangle=0.47 \pm 0.20 \mathrm{GeV}^{4} .
$$

However, a number of more recent investigations place the gluon condensate at much larger values [34 36]. For example, a finite-energy sum rule (FESR) analysis of the $\rho$-meson channel places the gluon condensate at 2 to 5 times the standard value [35]. In this analysis the spectral density is taken from a fit of the isospin-one channel of $e^{+} e^{-}$scattering data. First results from the ALEPH and CLEO II experiments [34,37] also suggest a larger value at $\left\langle\alpha_{s} / \pi G^{2}\right\rangle=0.025 \pm 0.010 \mathrm{GeV}^{4}$. A recent Laplace based sum rule analyses [38] suggests a value of $\left\langle\alpha_{s} / \pi G^{2}\right\rangle \simeq 0.015 \pm 0.005 \mathrm{GeV}^{4}$, more in accord with the standard value. However, we are somewhat skeptical of the refinements of this result as the analysis is based on an approach which is criticized in the following. T The most reliable sum rules of Sec. VII also prefer a larger gluon condensate in order to maintain consistency between the sum rules. There it is found that the nucleon mass may be reproduced with a value of $\left\langle\alpha_{s} / \pi G^{2}\right\rangle \simeq 0.03$ $\mathrm{GeV}^{4}$ which agrees with experimental estimate. Since the gluon condensate plays a critical role in the nucleon sum rules as part of a factorized dimension seven operator we adopt an uncertainty of $50 \%$ and take

$$
b=(2 \pi)^{2}\left\langle\frac{\alpha_{s}}{\pi} G^{2}\right\rangle=1.2 \pm 0.6 \mathrm{GeV}^{4} .
$$

The mixed condensate is parameterized as $\left\langle\bar{q} g_{c} \sigma \cdot G q\right\rangle=-m_{0}^{2}\langle\bar{q} q\rangle$. Early analyses of baryons [27] as well as more recent analyses of heavy-light quark systems [39] place

\footnotetext{
${ }^{4}$ For example, while the language used to describe the sum rules in Ref. [38] is somewhat different from that used here, the nature of the sum rules are the same. The concerns surrounding OPE convergence, continuum models and the use of derivative sum rules discussed in this paper still apply.
} 
$m_{0}^{2}=0.80 \mathrm{GeV}^{2}$, while an analysis of $N 3 / 2^{-}$QCD-SR sum rules demand $m_{0}^{2}$ to be smaller [9] at $m_{0}^{2}=0.65 \mathrm{GeV}^{2}$. Hence we take the average and half the difference as the uncertainty.

$$
m_{0}^{2}=0.72 \pm 0.08 \mathrm{GeV}^{2}
$$

Since the parameter $m_{0}^{2}$ is selected via Monte Carlo, the value of the mixed condensate is obtained by multiplying $m_{0}^{2}$ by the central value of the quark condensate, as opposed to a randomly selected value of the quark condensate. This is in contrast to factorized operators where both condensates appearing in the operator are selected via Monte Carlo.

Relatively little is known about the magnitude of the dimension-six, four-quark operators. Early arguments placed the values of these condensates within $10 \%$ of the factorized values [40. However other analyses claimed significant violation of factorization [10,34, 36, 38, 41, 42, for these operators in both nucleon and vector meson sum rules. Parameterizing the four quark-operators as $\kappa\langle\bar{q} q\rangle^{2}$, estimates of factorization violation place $\kappa=2$ or more. For the nucleon we consider

$$
\kappa_{N}=2 \pm 1, \quad \text { and } \quad 1.0 \leq \kappa_{N} \leq 4.0 .
$$

If one adopts the standard value for the gluon condensate, then $\kappa_{\rho} \sim \kappa_{N}$ is sufficient to reproduce the $\rho$-meson mass. However, selection of the more recent and reliable estimates of $b \sim 1.2 \mathrm{GeV}^{4}$ demands $\kappa_{\rho} \sim 6$ to reproduce the $\rho$-meson mass. This value is in accord with the observations of Refs. [34, 35], however the uncertainty on this parameter is large. Hence we adopt回

$$
\kappa_{\rho}=6 \pm 2, \quad \text { and } \quad 1.0 \leq \kappa_{\rho} \leq 10.0
$$

Figure 1 displays histograms drawn from 1000 QCD parameter sets for four of the QCD parameters discussed to this point.

Variation of the QCD scale parameter $\Lambda_{\mathrm{QCD}}^{(3)}$ has little effect on the results. However, we restrict the values to the conventional range adopted in QCD-SRs

$$
\Lambda_{\mathrm{QCD}}^{(3)}=0.15 \pm 0.04 \mathrm{GeV}, \quad \text { and } \quad 0.10 \mathrm{GeV} \leq \Lambda_{\mathrm{QCD}}^{(3)} \leq 0.20 \mathrm{GeV} .
$$

Variation of $\Lambda_{\mathrm{QCD}}^{(3)}$ above $200 \mathrm{MeV}$ is only sensible if the separation scale $\mu$ of the OPE, taken to be $500 \mathrm{MeV}$, is increased to maintain plausible convergence of the power corrections of the OPE. A change in $\mu$ redefines the condensate values. A systematic determination of the new condensate values is beyond the scope of this investigation and we choose to simply restrict the value as above.

It is perhaps interesting to note that if $\Lambda_{\mathrm{QCD}}^{(3)}$ is very large, say near the upper limit of the current world average [43], the OPE separation scale would necessarily become large. Consequently, the Borel mass would be restricted to larger values, and access to ground state

\footnotetext{
${ }^{5}$ With $\kappa_{\rho} \sim 6$ as opposed to 2 , even a small suppression of the chirally even four-quark condensate at finite density can give rise to substantial suppression of the $\rho$-meson mass and width. Further investigation of these issues is warranted.
} 

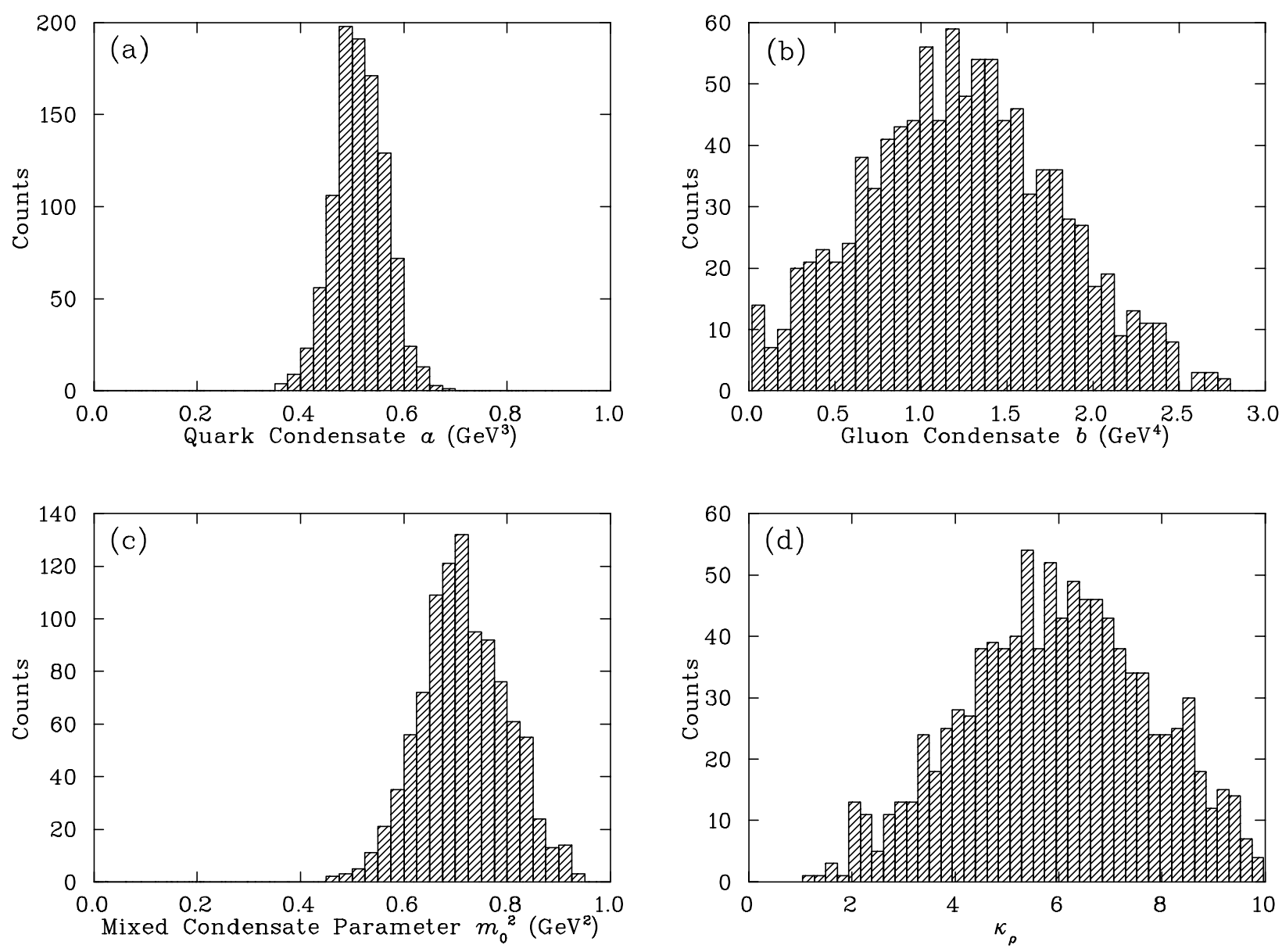

FIG. 1. Histograms for QCD parameter distributions drawn from a sample of 1000 QCD parameter sets. Included are (a) the quark condensate parameter of (3.1), (b) the gluon condensate parameter of (3.3), (c) the mixed condensate parameter $m_{0}^{2}$ and $(\mathrm{d})$ the vacuum saturation violation parameter $\kappa_{\rho}$.

properties may not be possible. The correlator may be dominated by strength from excited states, similar to the present situation for the pseudoscalar pion correlator. Fortunately, the lower bound on the current world average for $\Lambda_{\mathrm{QCD}}^{(3)}$ bodes well for present QCD sum rule analyses. However, if the current world average for $\Lambda_{\mathrm{QCD}}^{(3)}$ persists, a comprehensive reformulation of the approach should be pursued.

The $\rho$-meson sum rule requires knowledge of $\alpha_{s} / \pi$ at the scale of $1 \mathrm{GeV}^{2}$. Since this scale is rather low for two-loop perturbation theory, we adopt a $10 \%$ uncertainty

$$
\frac{\alpha_{s}\left(1 \mathrm{GeV}^{2}\right)}{\pi}=0.117 \pm 0.012 \text {. }
$$

Finally, the $\rho$-meson sum rule requires knowledge of the average light quark mass $m_{q}$. Since it appears multiplied by the quark condensate, we will use the relation of (2.25) and equate 


$$
m_{q}\langle\bar{q} q\rangle=-\frac{1}{2} m_{\pi}^{2} f_{\pi}^{2}
$$

Monte-Carlo techniques might also be used to probe OPE truncation errors by randomly selecting the size of the next term to follow the truncated series. The problem lies in determining a sensible and realistic estimate without actually calculating the term. Instead, we will emphasize the importance of OPE convergence and select the Borel analysis regime such that truncated terms are very unlikely to be important.

\section{QCD-SR ANALYSIS}

\section{A. Monte-Carlo Uncertainty Analysis}

Gaussian distributions are generated using established algorithms for the conversion of uniform distributions on the interval $(0,1)$ 43. A particularly fast algorithm used in this investigation is as follows. If $u_{1}$ and $u_{2}$ are uniform on $(0,1)$, then construct $v_{1}=2 u_{1}-1$ and $v_{2}=2 u_{2}-1$. Calculate $r^{2}=v_{1}^{2}+v_{2}^{2}$. If $r^{2}>1$ start over. Otherwise, calculate

$$
z_{1}=v_{1}\left(\frac{-2 \ln r^{2}}{r^{2}}\right)^{1 / 2}, \text { and } z_{2}=v_{2}\left(\frac{-2 \ln r^{2}}{r^{2}}\right)^{1 / 2} \text {. }
$$

$z_{1}$ and $z_{2}$ are independent and normally distributed with mean 0 and variance 1.

A set of Gaussianly-distributed randomly-selected condensate values is generated, from which an OPE in Borel space, $\Pi^{\mathrm{OPE}}(M)$, is constructed. By repeating this procedure, an uncertainty for the OPE may be determined. Selecting $n_{B}$ evenly distributed points in the Borel parameter space $\left(M_{j}, j=1,2, \ldots n_{B}\right)$, the standard deviation in the OPE at the $j$ 'th Borel mass is given by

$$
\sigma_{\mathrm{OPE}}^{2}\left(M_{j}\right)=\frac{1}{n_{C}-1} \sum_{i=1}^{n_{C}}\left[\Pi_{i}^{\mathrm{OPE}}\left(M_{j}\right)-\overline{\Pi^{\mathrm{OPE}}}\left(M_{j}\right)\right]^{2}
$$

where $i$ denotes the $i$ 'th set of $n_{C}$ QCD parameter sets and

$$
\overline{\Pi^{\mathrm{OPE}}}\left(M_{j}\right)=\frac{1}{n_{C}} \sum_{i=1}^{n_{C}} \Pi_{i}^{\mathrm{OPE}}\left(M_{j}\right) .
$$

In practice, $n_{C}=500$ was used in estimating the OPE uncertainty. However, $n_{C}=100$ was sufficient to get a stable uncertainty estimate.

The uncertainties in the OPE are not uniform throughout the Borel regime. They are larger at the lower nonperturbative end of the Borel region where uncertainties in the higherdimensional vacuum condensates dominate. Hence, it is crucial that the appropriate weight is used in the calculation of $\chi^{2}$. Having determined the OPE uncertainty, a $\chi^{2}$ measure is

easily constructed. For the OPE obtained from the $k^{\prime}$ th set of QCD parameters, the $\chi^{2}$ per degree of freedom is

$$
\frac{\chi_{k}^{2}}{N_{D F}}=\frac{1}{n_{B}-n_{p}} \sum_{j=1}^{n_{B}} \frac{\left[\Pi_{k}^{\mathrm{OPE}}\left(M_{j}\right)-\Pi^{\mathrm{SR}}\left(M_{j} ; \lambda_{k}, m_{k}, w_{k}\right)\right]^{2}}{\sigma_{\mathrm{OPE}}^{2}\left(M_{j}\right)},
$$


where $n_{p}$ is the number of phenomenological search parameters, and $\Pi^{\mathrm{SR}}\left(M_{j} ; \lambda_{k}, m_{k}, w_{k}\right)$ denotes the phenomenological Spectral Representation of the QCD-SR. In practice, $n_{B}=51$ points were used along the Borel axis. $\chi^{2}$ is minimized by adjusting the phenomenological parameters including the pole residue $\lambda_{\mathcal{O}}$, the hadron mass $m$, and the continuum threshold $w$.

Distributions for the phenomenological parameters are obtained by minimizing $\chi^{2}$ for many QCD parameter sets. Provided the resulting distributions are Gaussian, an estimate of the uncertainty in the phenomenological results such as the hadron mass is obtained from

$$
\sigma_{m}^{2}=\frac{1}{N-1} \sum_{k=1}^{N}\left(m_{k}-\bar{m}\right)^{2}, \quad \text { with } \quad \bar{m}=\frac{1}{N} \sum_{k=1}^{N} m_{k} .
$$

In the event that the resultant distribution is not Gaussian, we will report the median and asymmetric standard deviations from the median. We note that (4.5) is the standard deviation of the distribution and not the standard error obtained by further dividing by $N$, the number of QCD parameter sets in the sample. The standard deviation is roughly independent of the number of QCD parameter sets used in the analysis, and directly reflects the input parameter uncertainties. In the following, we generally select $N=1000$. While 100 QCD parameter configurations are sufficient for obtaining reliable uncertainty estimates, we also wish to explore correlations among the QCD parameters and the phenomenological fit parameters. The extra sets aid in resolving more subtle correlations.

Some care must be taken in the interpretation of the $\chi^{2} / N_{D F}$. While we are free to choose any number of points $n_{B}$ along the Borel axis for fitting the two sides of the sum rule, it is important to recognize that the actual number of degrees of freedom is determined by the number of terms in the OPE. For the $\chi^{2} / N_{D F}$ to have true statistical meaning, a correlated $\chi^{2}$ calculation is required.

$$
\begin{aligned}
& \chi_{k}^{2}=\sum_{j=1}^{n_{B}} \sum_{j^{\prime}=1}^{n_{B}}[\left.\Pi_{k}^{\mathrm{OPE}}\left(M_{j}\right)-\Pi^{\mathrm{SR}}\left(M_{j} ; \lambda_{k}, m_{k}, w_{k}\right)\right] \times \\
& C_{j j^{\prime}}^{-1}\left[\Pi_{k}^{\mathrm{OPE}}\left(M_{j^{\prime}}\right)-\Pi^{\mathrm{SR}}\left(M_{j^{\prime}} ; \lambda_{k}, m_{k}, w_{k}\right)\right],
\end{aligned}
$$

where $C_{j j^{\prime}}^{-1}$ is the inverse covariance matrix. The covariance matrix may be estimated by

$$
C_{j j^{\prime}}=\frac{1}{\left(n_{C}-1\right)} \sum_{i=1}^{n_{C}}\left[\Pi_{i}^{\mathrm{OPE}}\left(M_{j}\right)-\overline{\Pi^{\mathrm{OPE}}}\left(M_{j}\right)\right]\left[\Pi_{i}^{\mathrm{OPE}}\left(M_{j^{\prime}}\right)-\overline{\Pi^{\mathrm{OPE}}}\left(M_{j^{\prime}}\right)\right]
$$

Unfortunately, for most of the fits considered here the covariance matrix is ill-conditioned. Inverting the covariance matrix via singular value decomposition leads to well known pathological problems 44 such as best fit lines which do not pass through the data points etc. While one could experiment with different approaches to stepping around this problem, we prefer to use the robust method of determining best fit parameters by minimizing the uncorrelated $\chi^{2}$ of (4.4). Even if some correlation among the data is suspected, it is still acceptable to use the uncorrelated $\chi^{2}$ fit provided the errors on the parameters are estimated using a Monte-Carlo based procedure like that discussed here rather than from the dependence of $\chi^{2}$ on the parameters 44,45]. 
When simultaneously fitting multiple sum rules which may have some fit parameters in common, (4.4) is simply modified to

$$
\frac{\chi^{2}}{N_{D F}}=\frac{1}{n_{\mathrm{SR}} n_{B}-n_{p}} \sum_{i=1}^{n_{\mathrm{SR}}} \sum_{j=1}^{n_{B}} \frac{\left[\Pi_{i}^{\mathrm{OPE}}\left(M_{i j}\right)-\Pi_{i}^{\mathrm{SR}}\left(M_{i j} ; \lambda_{i}, m, w_{i}\right)\right]^{2}}{\sigma_{\mathrm{OPE} i}^{2}\left(M_{i j}\right)}
$$

where $i$ runs over the considered sum rules. (In this example, $m$ is a common parameter to all $n_{\mathrm{SR}}$ sum rules.) The subscript $i$ in $M_{i j}$ indicates that each sum rule has a unique set of $n_{B}$ Borel masses $M_{j}$ spanning the region of interest in Borel space. The subscript $k$ in (4.4), denoting a particular set of QCD parameters, has been suppressed. In the following discussion it will become apparent that it is advantageous to weight the contributions in the sum over the various sum rules by the size of their valid Borel regimes. If $V_{B}^{i}$ is the size of the valid Borel regime for the $i$ 'th sum rule, then (4.8) is modified to

$$
\frac{\chi^{2}}{N_{D F}}=\frac{1}{n_{\mathrm{SR}} n_{B}-n_{p}} \frac{n_{\mathrm{SR}}}{\sum_{i}^{n_{\mathrm{SR}}} V_{B}^{i}} \sum_{i=1}^{n_{\mathrm{SR}}} \sum_{j=1}^{n_{B}} \frac{V_{B}^{i}\left[\Pi_{i}^{\mathrm{OPE}}\left(M_{i j}\right)-\Pi_{i}^{\mathrm{SR}}\left(M_{i j} ; \lambda_{i}, m, w_{i}\right)\right]^{2}}{\sigma_{\mathrm{OPE} i}^{2}\left(M_{i j}\right)} .
$$

\section{B. Search Algorithm}

The optimization method utilized in this investigation is an updated version of a direction-set routine by Powell originally published in Ref. 46]. This algorithm finds the minimum value of a function by iterative variation of the function parameters which need not be independent. Modifications of the original routine improve the selection of conjugate directions in the search and avoid the possible generation of linearly dependent search directions 477.

Powell's method is among the best methods for finding minima in a multidimensional parameter space when derivatives are not readily available 48,49. Part of the reason for the robust nature of Powell's routine is the cautious criterion for ultimate convergence. Once a minimum is found, the optimal vector, $\vec{a}$, in the parameter space is displaced by ten times the requested accuracy. The search proceeds from this new point until a minimum is found at vector $\vec{b}$. The minimum on the line joining $\vec{a}$ and $\vec{b}$ is found at $\vec{c}$. Convergence is reached if the components of the vectors $(\vec{a}-\vec{c})$ and $(\vec{b}-\vec{c})$ are all less than $10 \%$ of the required accuracy. Otherwise the direction $(\vec{a}-\vec{c})$ is utilized in a new search.

The displacement of the first minimum by ten times the requested accuracy plays a crucial role in removing the sensitivity of the ultimate minimum from the initial parameter estimates. While there is sufficient flexibility in the algorithm parameters to search in the locality of the initial parameter estimates, the opposite criteria has been utilized in this investigation. One of the main goals of this investigation is to eliminate the possibility of fine tuning the initial parameter estimates which otherwise can bias the results. Instead, we are interested in the predictions of the QCD sum rules. 


\section{V. $\rho$-MESON SUM RULES}

The fundamental QCD-SR for the $\rho$ meson follows from the consideration of (2.1), (2.2), (2.12) and (2.29). We consider the correlator, $\Pi_{S}\left(M^{2}\right)$, where the continuum model is subtracted from the phenomenological side and placed on the OPE side.

$$
\begin{aligned}
\Pi_{S}\left(M^{2}\right)=f_{\rho}^{2} e^{-M_{\rho}^{2} / M^{2}}= & c_{0} M^{2}\left[1-e^{w_{\rho}^{2} / M^{2}}\right]+c_{1}+\frac{c_{2}}{M^{2}}+\frac{c_{3}}{2 ! M^{4}}+\cdots \\
& +\frac{c_{m}}{(m-1) !\left(M^{2}\right)^{m-1}}+\cdots
\end{aligned}
$$

The Wilson coefficients are given by [1]

$$
\begin{aligned}
c_{0} & =\frac{1}{8 \pi^{2}}\left(1+\frac{\alpha_{s}}{\pi}\right), \quad c_{1}=0, \\
c_{2} & =m_{q}\langle\bar{q} q\rangle+\frac{1}{24}\left\langle\frac{\alpha_{s}}{\pi} G^{2}\right\rangle, \\
c_{3} & =-\frac{112}{81} \pi \alpha_{s} \kappa_{\rho}\langle\bar{q} q\rangle^{2} .
\end{aligned}
$$

The phenomenology of the QCD-SR is described by the vector-meson pole of interest plus the continuum model accounting for the contributions of all excited states. By working in a region where the pole dominates the phenomenology, one can minimize sensitivity to the model and have assurance that it is the spectral parameters of the ground state of interest that are being determined by matching the sum rules. In practice, these considerations effectively set an upper limit in the Borel parameter space, beyond which the model for excited states dominates the phenomenological side.

At the same time, the truncated OPE must be sufficiently convergent as to accurately describe the true OPE. Since the OPE is an expansion in the inverse squared Borel mass, this consideration sets a lower limit in Borel parameter space, beyond which higher order terms not present in the truncated OPE are significant and important. Monitoring OPE convergence is absolutely crucial to recovering nonperturbative phenomena in the sum-rule approach, as it is the lower end of the Borel region where the nonperturbative information of the OPE is most significant. As we shall see, this information must also be accurate.

In short, one should not expect to extract information on the ground state spectral properties unless the ground state dominates the contributions on the phenomenological side and the OPE is sufficiently convergent. In this investigation, we will analyze each individual sum rule with regard to the above criteria. A sum rule with an upper limit in Borel space lower than the lower limit is considered invalid. As a measure of the relative reliability of various sum rules we consider the size of the regime in Borel space where both sides of the sum rules are valid. This quantity is used as a weight in the calculation of the $\chi^{2} / N_{D F}$ as indicated in (4.9). In addition, the size of continuum contributions throughout the Borel region can also serve as a measure of reliability, with small continuum model contributions being more reliable.

The fiducial Borel region is chosen [9] such that the highest-dimensional operator(s) (HDO) contribute no more than $\sim 10 \%$ to the QCD side while the continuum contribution is less than $\sim 50 \%$ of the total phenomenological side (i.e., the sum of the pole and the 
continuum contribution). The former sets a criterion for the convergence of the OPE while the latter controls the continuum contribution. While the selection of $50 \%$ is obvious for pole dominance, the selection of $10 \%$ is a reasonably conservative criterion that has not failed in practice.9 See Ref. [6] for further specific examples.

Figure 2 displays the valid Borel window for the $\rho$-meson sum rule of (5.1). The HDO contributions limited to $10 \%$ of the OPE and the continuum model contributions limited to $50 \%$ of the phenomenology are illustrated. The continuum contributions are the order of $15 \%$ of the phenomenological contributions at the lower end of the Borel regime. Here, the pole of interest truly dominates the QCD-SR. Figure 3 displays the corresponding fit obtained by minimizing $\chi^{2}$ in a three parameter search including $f_{\rho}, M_{\rho}$ and $w_{\rho}$. Figure Ð displays the distribution of $\rho$-meson masses obtained from the 1000 sum rule fits. The distribution corresponds to

$$
M_{\rho}=0.76_{-0.16}^{+0.12} \mathrm{GeV} .
$$

The uncertainty is somewhat larger than the commonly assumed 10\%. Figure 5 displays the distribution of the square of $f_{\rho}$ which corresponds to

$$
f_{\rho}=0.144 \pm 0.021 \mathrm{GeV},
$$

which agrees with the experimental value of $f_{\rho}=M_{\rho} / g_{\rho}=0.153 \mathrm{GeV}$. The continuum threshold is $w_{\rho}=1.23 \pm 0.17 \mathrm{GeV}$ which compares favorably with $1.31 \pm 0.04 \mathrm{GeV}$ (the $\rho^{\prime}$ mass less the half width), given the approximate nature of the continuum model.

\section{A. Derivative Sum Rules and the Ratio Method}

In addition to the fundamental sum rule of (5.1) one also finds the use of sum rules obtained by differentiating (5.1) with respect to $1 / M^{2}$. For the $n$ 'th derivative

$$
\begin{aligned}
f_{\rho}^{2}\left(M_{\rho}^{2}\right)^{n} e^{-M_{\rho}^{2} / M^{2}}= & n ! c_{0}\left(M^{2}\right)^{n+1} E_{n}+(-1)^{n}\left[c_{n+1}+\frac{c_{n+2}}{M^{2}}+\cdots\right. \\
& \left.+\frac{c_{m}}{(m-n-1) !\left(M^{2}\right)^{m-n-1}}+\cdots\right]
\end{aligned}
$$

While these sum rules are obviously unnecessary for the extraction of $\rho$-meson properties, the hope is that these additional sum rules will provide new useful information that might be used to further constrain the phenomenological properties.

It is possible to isolate the $\rho$-meson mass as a function of the Borel mass, by taking a ratio of the first derivative sum rule with the fundamental sum rule. This approach has some aesthetic appeal, and has widely become the method of choice for analyzing QCDSRs. The continuum threshold is selected to make the ratio of the two sum rules as flat as

\footnotetext{
${ }^{6}$ Reasonable alternatives to the $10 \%$ and $50 \%$ criteria are automatically explored in the MonteCarlo error analysis, as the condensate values and the continuum threshold change in each sample.
} 


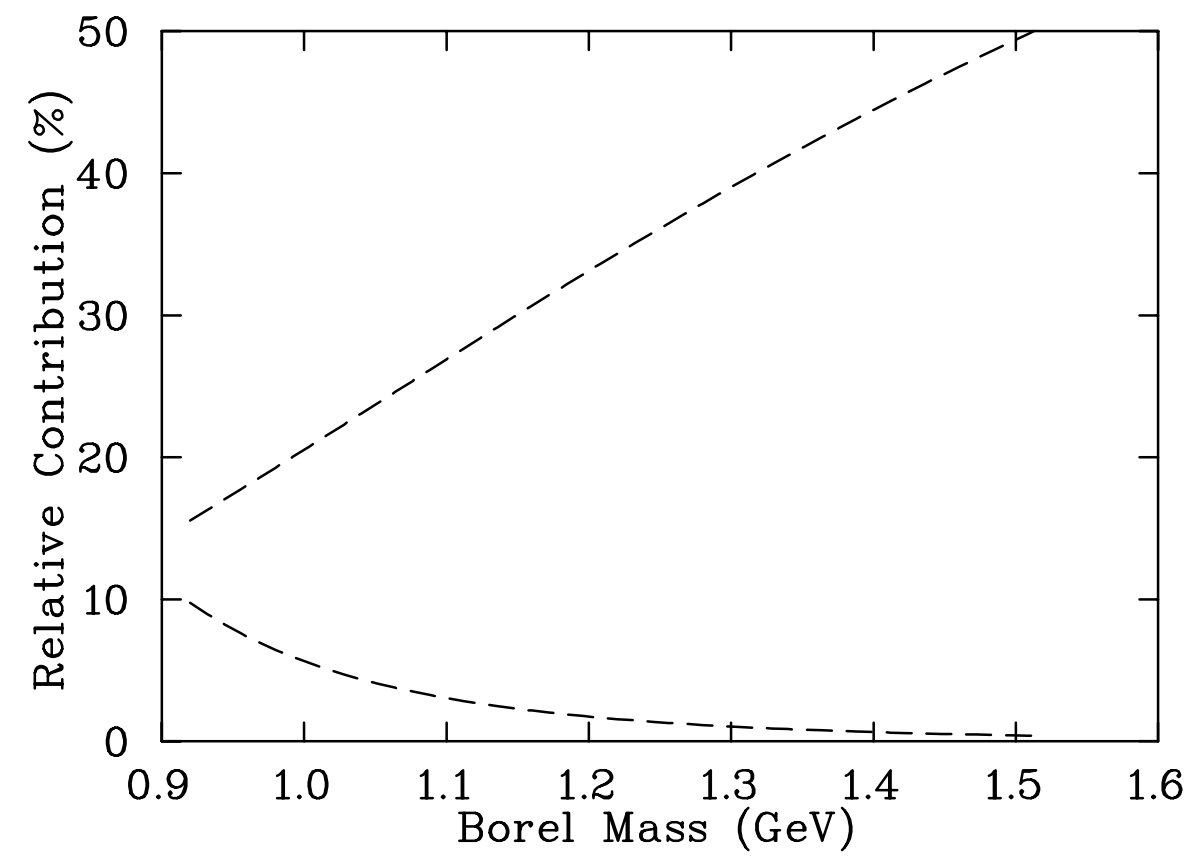

FIG. 2. The valid Borel window for the $\rho$-meson sum rule of (5.1). The relative HDO contributions limited to $10 \%$ of the OPE and the continuum model contributions limited to $50 \%$ of the phenomenology are illustrated.

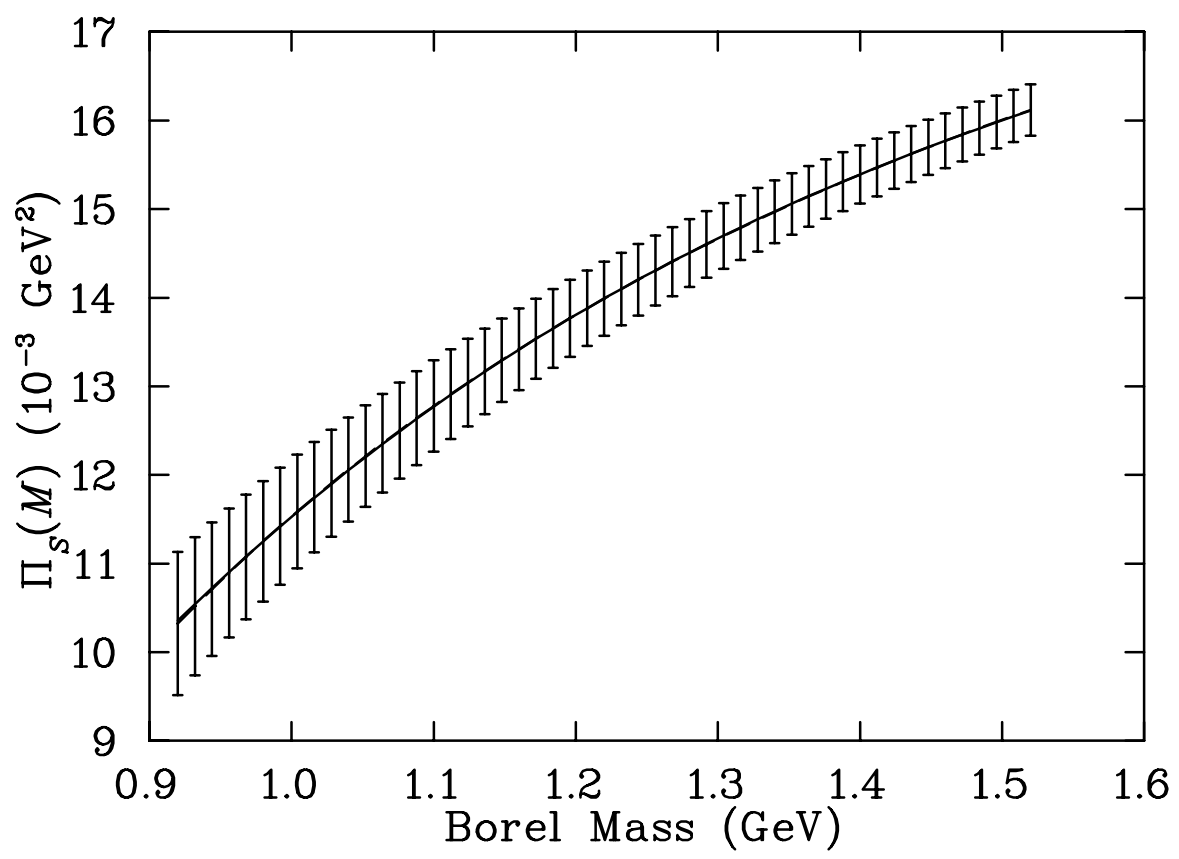

FIG. 3. A three parameter fit including $f_{\rho}, M_{\rho}$ and $w_{\rho}$ of the $\rho$-meson sum rule of (5.1) for the central values of the condensate distributions. The error bars illustrate the OPE uncertainty estimated at each of the 51 points considered in the $\chi^{2}$ estimate. Both the QCD-continuum (dashed) and pole (solid) sides of (5.1) are plotted. 


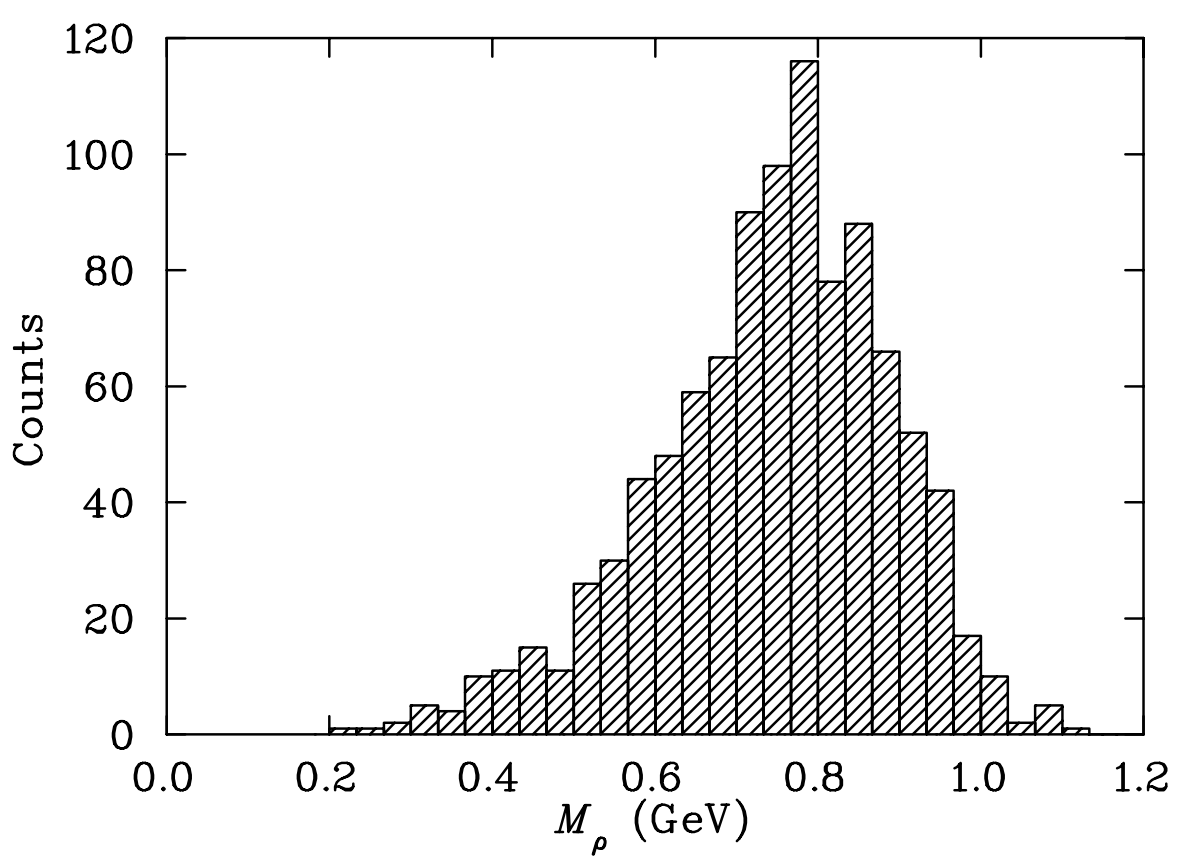

FIG. 4. Histogram of $\rho$-meson masses obtained from 1000 fits of (5.1) for 1000 QCD parameter sets. The distribution corresponds to $M_{\rho}=0.76_{-0.16}^{+0.12} \mathrm{GeV}$.

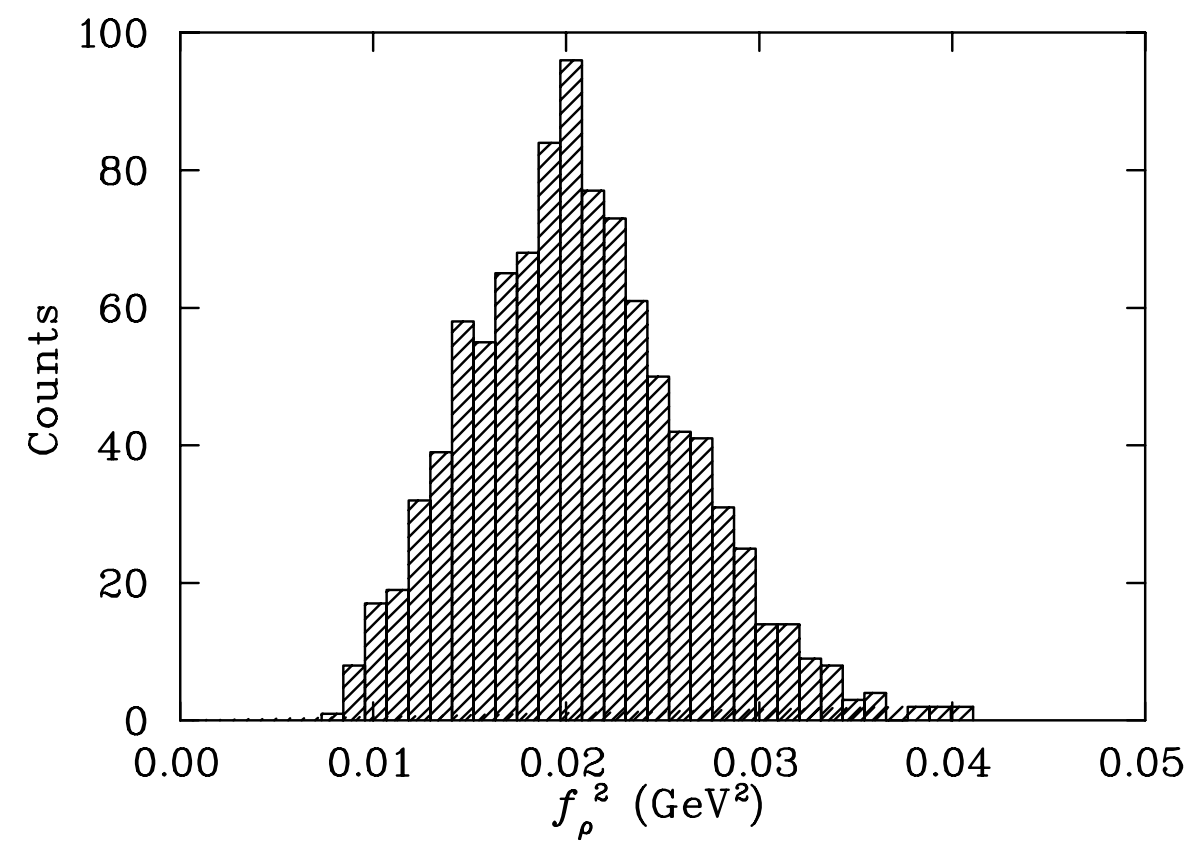

FIG. 5. Histogram of the squared $\rho$-meson decay constant obtained from 1000 fits of (5.1) for 1000 QCD parameter sets. The distribution corresponds to $f_{\rho}^{2}=0.021 \pm 0.006 \mathrm{GeV}^{2}$. 
possible as a function of Borel mass. Finally, the $\rho$-meson mass is selected from the point at which the ratio is most flat or stable. Unfortunately, this method has some very unpleasant shortcomings.

The ratio method does not check the validity of each individual sum rule. It is possible to have individual sum rules that are not valid while their ratio is flat as function of Borel mass. In addition, the ratio method cannot account for the fact that sum rules do not work equally well. The Borel region where a sum rule is valid can vary from one sum rule to another. Moreover, large uncertainties in one sum rule can spoil the predictions of another sum rule. Finally, the continuum contributions to the sum rules are not monitored in the ratio method. If the continuum contribution is dominant in a sum rule, one should not expect to get reliable information about the lowest resonance.

The fact that sum rules do not work equally well deserves further discussion. In Ref. [6] a detailed comparison of fundamental and derivative sum rules is carried out for the $\rho$ meson. These arguments are not broadly known and are generally applicable to any derivative Borel-improved QCD-SR. Hence the important observations are highlighted here.

If one could carry out the OPE to arbitrary accuracy and use a spectral density independent of models for excited state contributions, the predictions based on (5.1) and those based on the derivative sum rules of (5.5) should be the same. In practical calculations, however, one has to truncate the OPE and use a simple phenomenological ansatz for the spectral density. Thus it is unrealistic to expect the sum rules to work equally well. Compare the $n$ 'th derivative sum rule of (5.5) with the direct sum rule of (5.1).

1. The perturbative contribution in the derivative sum rule has an extra factor $n$ ! relative to the corresponding term in (5.1), implying that the perturbative contribution is more important in the derivative sum rules than in the fundamental sum rule, and becomes increasingly important as $n$ increases. Since the perturbative term mainly contributes to the continuum of the spectral density, maintaining dominance of the lowest resonance pole in the sum rule will become increasingly difficult as $n$ increases.

2. In (5.1), the term proportional to $c_{m}$ is suppressed by a factor of $1 /(m-1)$ !, while it is only suppressed by $1 /(m-n-1)$ ! in the derivative sum rule $(m>n)$. This implies that the convergence of the OPE is much slower in the derivative sum rule than in the fundamental sum rule. Consequently, the poorly known high-order power corrections are more important in the derivative sum rule than in Eq. (5.1), and become more and more important as $n$ increases. If one would like to restrict the size of the last term of the OPE to maintain some promise of OPE convergence, the size of the Borel region in which the sum rules are believed to be valid is restricted.

3. The power corrections proportional to $c_{1}, c_{2}, \cdots, c_{n}$ do not contribute to the $n^{\text {th }}$ derivative sum rule but do contribute to (5.1). If one truncates the OPE, part or all of the nonperturbative information will be lost in the derivative sum rules. It is also worth noting that the leading power corrections are the most desirable terms to have. They do not give rise to a term in the continuum model and they are not the last term in the OPE, whose relative contribution should be restricted to maintain OPE convergence.

In practice, the predictions based on the fundamental sum rule of (5.1) are more reliable 
than those from the derivative sum rules, which become less and less reliable as $n$ increases. This can also be demonstrated by analyzing the sum rules numerically.

Figure 6 displays the valid Borel windows for a simultaneous fit of the $\rho$-meson sum rules of (5.1) and the first derivative $(n=1)$ of (5.5). The reliability of the fundamental sum rule over the derivative sum rule is obvious. The valid Borel regime for the derivative sum rule is nearly nonexistent.

All three points of the discussion surrounding derivative sum rules are displayed in this figure. Item 1 indicates the continuum model contributions will be much larger in the derivative sum rule for a given Borel mass. Indeed the continuum model contribution never drops below $45 \%$ in the valid regime. The relative contribution of the last term in the truncated OPE is larger in the derivative sum rule for a given Borel mass, in agreement with the expectations of items 2 and 3 .

Figure 7 displays the corresponding fit obtained by minimizing the weighted $\chi^{2} / N_{D F}$ of (4.9) in a three parameter search including $f_{\rho}, M_{\rho}$ and $w_{\rho}$. This time the logarithms of the left- and right-hand sides of equations (5.1) and (5.5) are plotted as a function of the inverse squared Borel mass. This method has the advantage of revealing the possible breakdown of the OPE signified by deviations of the dashed curves from linearity. Uncertainties are displayed only at the ends of the Borel region for clarity.

While the central values of the best fit parameters $M_{\rho}=0.77 \pm 0.07 \mathrm{GeV}, f_{\rho}^{2}=0.022 \pm$ $0.002 \mathrm{GeV}^{2}$, and $w_{\rho}=1.31 \pm 0.05 \mathrm{GeV}$, remain essentially unchanged, the reduction of the uncertainties comes from rather unreliable information as discussed above. These arguments are further supported by the failure of the two sides of (5.5) for $n=1$ to agree as illustrated in figure 7. To further probe the reasons for this failure, the analysis of these sum rules is repeated with a spectral representation that accounts for the finite width of the $\rho$ meson.

To account for the finite width of the $\rho$-meson, the $\delta$ function in (2.12) is replaced by a normalized Breit-Wigner form

$$
\frac{1}{\pi} \frac{M_{\rho} \Gamma_{\rho}}{\left(s-M_{\rho}^{2}+\Gamma_{\rho}^{2} / 4\right)^{2}+M_{\rho}^{2} \Gamma_{\rho}^{2}},
$$

and the integral over the $\rho$-meson ground state

$$
I_{\rho}\left(M^{2}\right)=\frac{M_{\rho} \Gamma_{\rho}}{\pi} \int_{4 m_{\pi}^{2}}^{\infty} \frac{d s e^{-s / M^{2}}}{\left(s-M_{\rho}^{2}+\Gamma_{\rho}^{2} / 4\right)^{2}+M_{\rho}^{2} \Gamma_{\rho}^{2}},
$$

is handled numerically [7]. Using the experimental width of $151.5 \mathrm{MeV}$, a three parameter fit of the fundamental and derivative sum rules with the Breit-Wigner form leads to essentially the same results reported in figure 7. The mass, coupling, and continuum threshold all agree within uncertainties, and have similar uncertainties. Hence, these spectral properties may be reliably estimated without precise information on the $\rho$ width. However, $\rho-\omega$ mixing provides an interesting example where finite mesonic widths can play a central role in arriving at the correct physics [7,50].

The origin of the discrepancy for the derivative sum rule is more likely to lie in shortcomings of continuum model. For this sum rule the continuum model contributions never drop below 45\%, which may be too large for the simple model typically used in QCD-SR calculations. Alternatively, poorly known higher order terms, not present in the truncated 


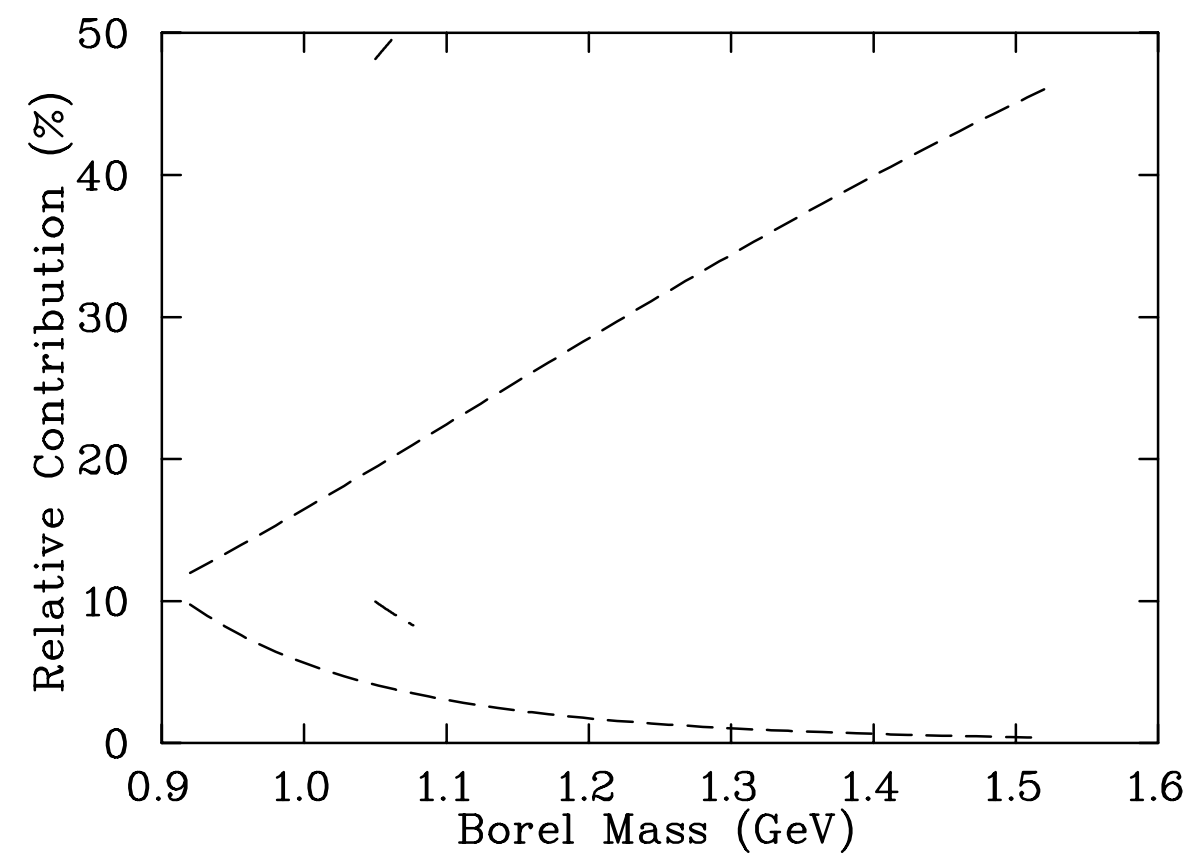

FIG. 6. The valid Borel windows for the fundamental $\rho$-meson sum rule of (5.1) (dashed) and the first derivative sum rule of (5.5) (dot-dashed). Both the relative HDO contributions limited to $10 \%$ and the continuum model contributions limited to $50 \%$ are illustrated.

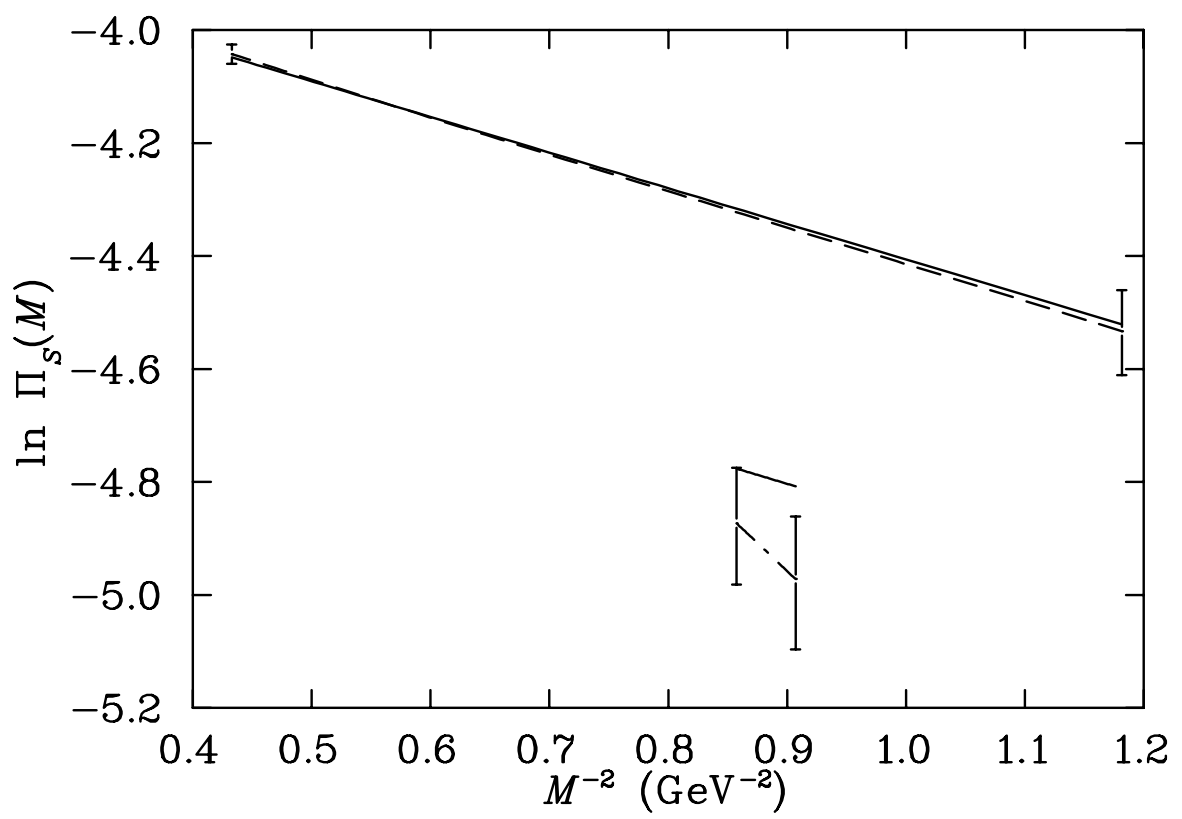

FIG. 7. A three parameter fit including $f_{\rho}, M_{\rho}$ and $w_{\rho}$ of the fundamental $\rho$-meson sum rule of (5.1) (dashed) and the first derivative sum rule of (5.5) (dot-dashed) for the central values of the condensate distributions. Solid lines indicate the ground state contributions to these sum rules. In this and the following plots displaying sum rule fits, uncertainties are displayed only at the ends of the Borel region for clarity. 
OPE may become important in the derivative sum rule. In the derivative sum rule, the next term in the series is suppressed by merely 2 ! .

The importance of carefully monitoring the validity of QCD-SRs is nicely displayed in the recent debate over the behavior of vector-meson masses in nuclear matter. There an analysis involving invalid sum rules leads to the conclusion that vector-meson masses increase in finite density [51], in contradiction to a previous analysis [52] also based on the ratio method. The debate is resolved in Ref. [6] where valid sum rules indicate that vector-meson masses decrease for increasing nuclear matter density.

Having firmly established the analysis procedure in the well understood $\rho$-meson channel, we are ready to apply the same approach to the nucleon sum rules. We will find that many results contradict the conventional wisdom.

\section{NUCLEON SUM RULES: SPIN-1/2 INTERPOLATORS}

Here we focus on the Borel improved QCD Sum Rules for the generalized interpolator of (2.3). The sum rules proportional to the correlator $\Pi_{S}(M)$ where continuum model contributions are subtracted from the phenomenological side and placed on the OPE side are

$$
\begin{gathered}
(\gamma \cdot p): \quad \frac{5+2 \beta+5 \beta^{2}}{64} M^{6} L^{-4 / 9}\left[1-e^{-w_{1}^{2} / M^{2}}\left(\frac{w_{1}^{4}}{2 M^{4}}+\frac{w_{1}^{2}}{M^{2}}+1\right)\right] \\
+\frac{5+2 \beta+5 \beta^{2}}{256} b M^{2} L^{-4 / 9}\left[1-e^{-w_{1}^{2} / M^{2}}\right] \\
+\frac{7-2 \beta-5 \beta^{2}}{24} a^{2} L^{4 / 9}-\frac{13-2 \beta-11 \beta^{2}}{96} \frac{m_{0}^{2} a^{2}}{M^{2}} L^{-2 / 27} \\
=\widetilde{\lambda}_{\mathcal{O}}^{2} e^{-M_{N}^{2} / M^{2}}
\end{gathered}
$$

and

$$
\begin{gathered}
(1): \frac{7-2 \beta-5 \beta^{2}}{16} a M^{4}\left[1-e^{-w_{2}^{2} / M^{2}}\left(\frac{w_{2}^{2}}{M^{2}}+1\right)\right] \\
-\frac{3\left(1-\beta^{2}\right)}{16} m_{0}^{2} a M^{2} L^{-14 / 27}\left[1-e^{-w_{2}^{2} / M^{2}}\right] \\
+\frac{19+10 \beta-29 \beta^{2}}{2^{7} 3^{2}} a b \\
=\widetilde{\lambda}_{\mathcal{O}}^{2} M_{N} e^{-M_{N}^{2} / M^{2}},
\end{gathered}
$$

where the Dirac- $\gamma$ structures are indicated on the left, and $\widetilde{\lambda}_{\mathcal{O}}=(2 \pi)^{2} \lambda_{\mathcal{O}}$. The factors of $L=\left[\log \left(M / \Lambda_{\mathrm{QCD}}\right) / \log \left(\mu / \Lambda_{\mathrm{QCD}}\right)\right]$ account for the anomalous scaling of the operators and interpolators in the leading logarithmic approximation [53].

\section{A. Traditional QCD-SR Analysis}

Let us begin with the traditional or standard nucleon sum rule analysis where $\alpha_{s}$ corrections are ignored. The more common interpolator of (2.5) is utilized by setting $\beta=-1$ in 
(6.1). In addition, the traditional condensate parameter set with $b=0.47 \pm 0.20 \mathrm{GeV}^{4}$ is selected in order to evaluate the validity of previously published results. For a preliminary analysis of the validities of the sum rules, the nucleon mass is fixed to $1 \mathrm{GeV}$. Figure 8 illustrates the valid Borel regimes for these two sum rules found by implementing the procedure discussed for the $\rho$-meson.

A comparison of figure 8 with the analogous figure 6 for the $\rho$-meson suggests the nucleon sum rules will not be as reliable. In the nucleon sum rules, the continuum model contributes $20 \%$ or more to the phenomenological side of the sum rules. In particular, the continuum model contribution in the sum rule of (6.1a) exceeds that for the derivative $\rho$-meson sum rule which has already been shown to be incompatible with the fundamental sum rule. It should not be surprising to find a similar disaster for the nucleon case.

As in the $\rho$-meson case, there is an analogous ratio method for obtaining the nucleon mass that has also become popular. Here the nucleon mass is isolated as a function of the Borel mass by taking the ratio of equations $(6.1 \mathrm{~b}) /(6.1 \mathrm{a})$. The mass is usually extracted from a regime in which the ratio is flat. Again OPE convergence and ground-state dominance criteria are not checked in this approach, and too often the mass is extracted from a regime in which the continuum model dominates.

Figure 9 illustrates the three parameter fit of the residue $\lambda_{\mathcal{O}}$, and the two continuum model thresholds of $w_{1}$ and $w_{2}$. The small Borel regime and the relatively huge OPE uncertainties of the sum rule of (6.1a) indicate that this sum rule plays a negligible role in determining the spectral properties of the nucleon. This is a perfect example of a case where large uncertainties in the sum rule of (6.1a) would spoil the predictive ability of (6.1b) if the ratio method was used. The origin of the large uncertainties lies in the poorly known value of the four-quark condensate. The resultant fit parameters for this sum rule are also unphysical as the continuum threshold lies at zero below the nucleon pole. In other words, the present form of the sum rule at $(\gamma \cdot p)$ is unable to provide any information on the ground state that is consistent with the more reliable sum rule of (6.1b) at the structure 1.

Unfortunately, the community has incorrectly identified (6.1a) as the more reliable sum rule. This belief is based on the simple fact that the leading term of the OPE (the identity operator) has a well known vacuum expectation value. However, the $\alpha_{s}$ corrections for the identity operator [54] are uncomfortably large. The first order corrections are independent of $\beta$ and are

$$
\frac{\alpha_{s}}{\pi}\left(\frac{53}{12}+\gamma_{E}\right)
$$

where $\gamma_{E}=\psi(1) \simeq 0.58$. This correction is $\sim 50 \%$ and suggests perturbative corrections are completely out of control for this sum rule. Since the magnitude of the identity operator contribution is closely tied to the size of continuum model contributions, it is not surprising to find the valid Borel regime for this sum rule disappears when $\alpha_{s}$ corrections are added.

Another factor in the traditional preference of (6.1a) over (6.1b) is that the second term of (6.1b) vanishes for Ioffe's choice of nucleon interpolator $(\beta=-1)$. This is really nothing more than a good reason to exclude the interpolator $\chi_{\mathrm{SR}}$ of (2.5) as an optimal interpolator.

When one considers the monstrous leading-order $\alpha_{s}$ corrections, the possibility of a significant dimension-two power correction arising from a summation of the perturbative series [25,21], and the large uncertainties associated with the value of the four-quark condensate, 


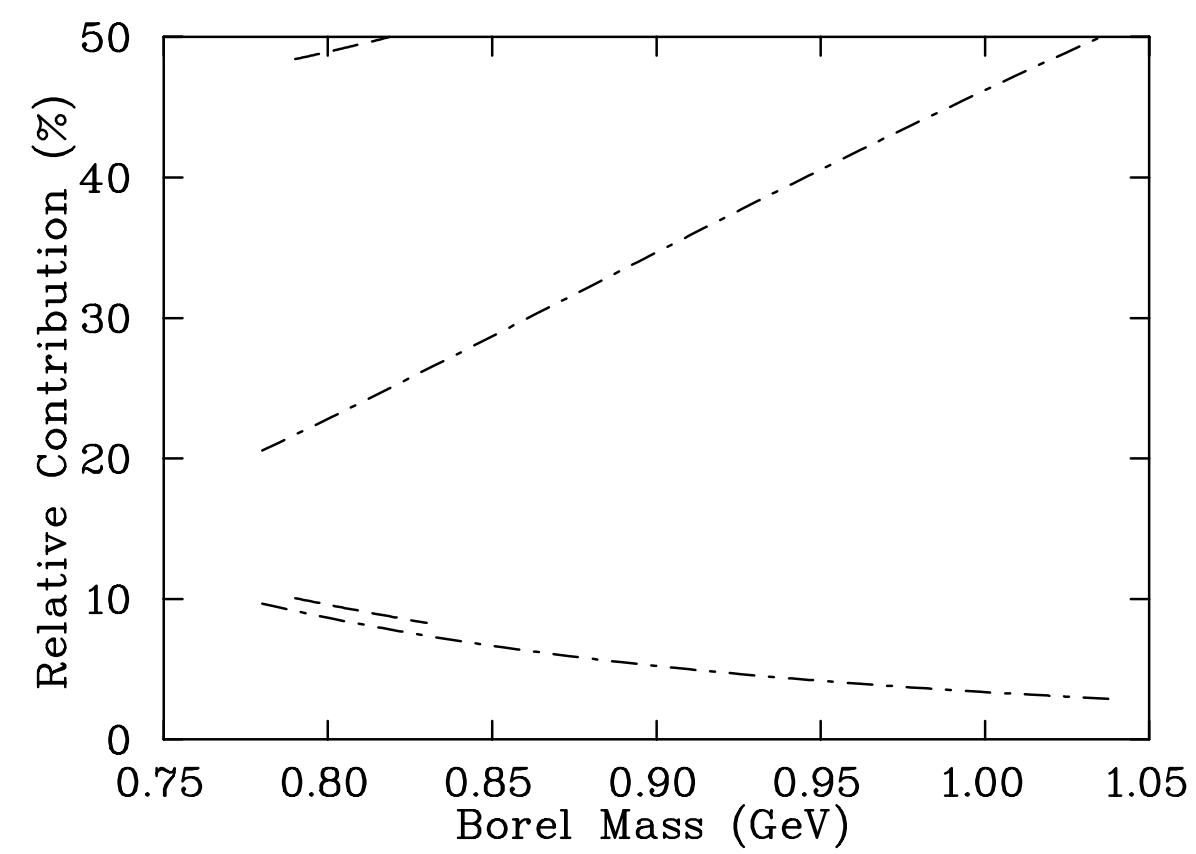

FIG. 8. The valid Borel windows for the nucleon sum rules of (6.19) (dashed) and (6.1b) (dot-dashed). Both the relative HDO contributions limited to $10 \%$ of the OPE and the continuum model contributions limited to $50 \%$ of the phenomenology are illustrated.

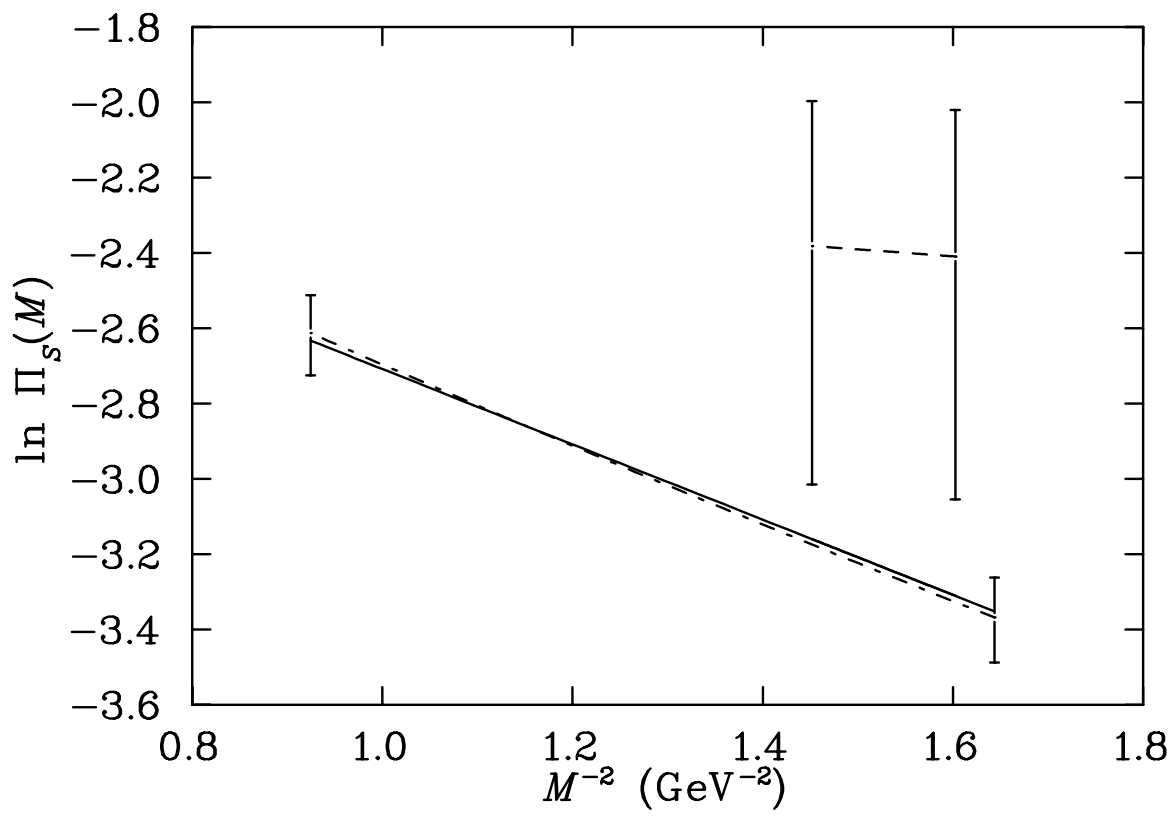

FIG. 9. Three parameter fit of (6.1a) (dashed) and (6.1b) (dot-dashed) to the ground state contribution (solid) for the residue $\lambda_{\mathcal{O}}$, and the two continuum model thresholds of $w_{1}$ and $w_{2}$. The small Borel regime and the relatively huge OPE uncertainties of the sum rule of (6.1a) indicate that this sum rule plays a negligible role in determining the spectral properties of the nucleon. 
one cannot help but reach the conclusion that the QCD side of the sum rule of (6.1a) is really unknown.

\section{B. Nucleon Sum Rule at $\gamma \cdot p$}

Since the sum rule of (6.1a) has traditionally been the nucleon sum rule of choice, it is interesting to consider the predictions of this sum rule alone. Applying the same techniques as used for the $\rho$-meson to (6.1a), a three parameter fit of $\lambda_{\mathcal{O}}, w_{1}$ and $M_{N}$ yields

$$
\tilde{\lambda}_{\mathcal{O}}^{2}=0.15 \pm 0.05 \mathrm{GeV}^{6}, \quad w_{1}=0.74 \pm 0.19 \mathrm{GeV}, \quad M_{N}=0.44 \pm 0.05 \mathrm{GeV}
$$

While setting $\kappa_{N}=1$ slightly increases the nucleon mass to $0.47 \mathrm{GeV}$, adding leading order $\alpha_{s}$ corrections has a negligible effect on the fit parameters. Consideration of the more recent condensate parameter set discussed in Sec. III leads to similar results

$$
\tilde{\lambda}_{\mathcal{O}}^{2}=0.14 \pm 0.10 \mathrm{GeV}^{6}, \quad w_{1}=0.71 \pm 0.5 \mathrm{GeV}, \quad M_{N}=0.43 \pm 0.09 \mathrm{GeV}
$$

This may be the first QCD sum rule to predict spectral properties that significantly contradict experiment. As such, this may be the most interesting of all QCD sum rules, and we shall return to this sum rule after examining the selection of the most optimal interpolator for nucleon sum rule analyses.

The predictions of (6.1a) summarized in (6.3) are extremely different from that found in the literature. Hence it is important to understand how these new results come about. A critical examination of the nucleon ground state results reported in Ref. 99 by the present author sheds considerable light on the importance of the rigorous QCD-SR analysis presented here.

Ref. [9] was the first analysis to give careful regard to the issues of OPE convergence and ground-state dominance of the phenomenological side. Moreover, all phenomenological parameters were included as fit parameters to be determined by the sum rules. However, there are three important differences between the analysis presented here and that of Ref. [9].

1. The standard logarithmic measure [27] of sum rule agreement was used to fit the sum rules. This approach fails to apply an appropriate weight to sum rule discrepancies, unlike the $\chi^{2}$ measure of 4.4$)$.

2. The algorithm used to fit the sum rules did not employ the cautious convergence criteria discussed in Section IVB. Implementation of the new criteria aids in avoiding insignificant local minima.

3. Only the standard condensate parameter set was considered in determining the nucleon mass. Here 1000 sets are considered.

\footnotetext{
${ }^{7}$ Note that this is unlike the case of the pseudoscalar pion current [55] where a check of OPE convergence and ground state dominance indicates the absence of a valid Borel regime.
} 
The optimal parameter set found in Ref. [9] corresponds to a local minimum which is sensitive to the measure of sum rule agreement, the initial parameter estimates and the condensate parameter set.

Other authors have managed to keep the nucleon mass closer to the experimental result by extracting the result from a regime in Borel space that provides the correct answer or fixing the continuum threshold to values suggested by the particle data tables. As we shall see in Section VIII, fixing the continuum threshold to phenomenologically motivated values largely determines the mass. Hence you can get anything you want provided you are clever enough to fix the threshold to the right value. And since the excited states are modeled there is tremendous freedom in the threshold parameter space. Of course, such input may not be in agreement with the content of the OPE. In any event, such results must not be regarded as predictions of QCD sum rules.

\section{Optimal Mixing of Spin-1/2 Interpolators}

Having established the nucleon sum rule of (6.1b) at the structure 1 to be the potentially more reliable sum rule, it is important to determine the optimal mixing of spin- $1 / 2$ interpolators for this sum rule.

It has long been established that there are two independent interpolating fields with no derivatives having the quantum numbers of spin $1 / 2$ and isospin $1 / 2$. Both are expected to excite the ground state nucleon from the vacuum. As such, there is a history of argument over the optimal nucleon interpolating field to be used in analyses , 9, 10, 12, 15,56, 58 and this issue is recognized to be of paramount importance [59].

While some advocate an interpolating field for which the leading terms of the OPE are stationary with respect to the interpolating field mixing parameter [10,58], others argue that a balance between OPE convergence and QCD continuum contributions must be maintained [9, 15,56,57]. Ideally, one would like to simply calculate with alternate interpolating fields and confirm that the nucleon properties remain unchanged. However, previous implementations of the QCD-SR approach have hindered any firm conclusions. The rigorous analysis introduced here will shed considerable light on these issues.

Fortunately, the lattice approach to QCD is not plagued with the same limitations and the debate over the optimal interpolating field was resolved in Ref. [13]. There is was found that, to a good approximation, $\chi_{2}$ of (2.4B) excites pure QCD continuum. Since $\chi_{2}$ has negligible overlap with the ground state nucleon, it is tempting to simply conclude that the optimal interpolating field is $\chi_{1}$. While this is certainly the case for lattice QCD investigations, it is not the case for QCD-SR analyses. By including $\chi_{2}$ components in an interpolating field, one can reduce the continuum contributions excited by $\chi_{1}$ and allow a broader Borel analysis window in which better contact with the ground state of interest is obtained.

One of the most difficult things to monitor in the QCD-SR approach is whether the OPE is sufficiently convergent for a particular value of Borel mass. The lattice results of Ref. [13] indicate the $\chi_{2} \bar{\chi}_{2}$ correlator has the fastest converging OPE, as its overlap with the nucleon ground state is negligible. Similarly, the combination $\chi_{1} \bar{\chi}_{1}$ produces an OPE

with the slowest convergence, as this correlation function is dominated by the ground state 


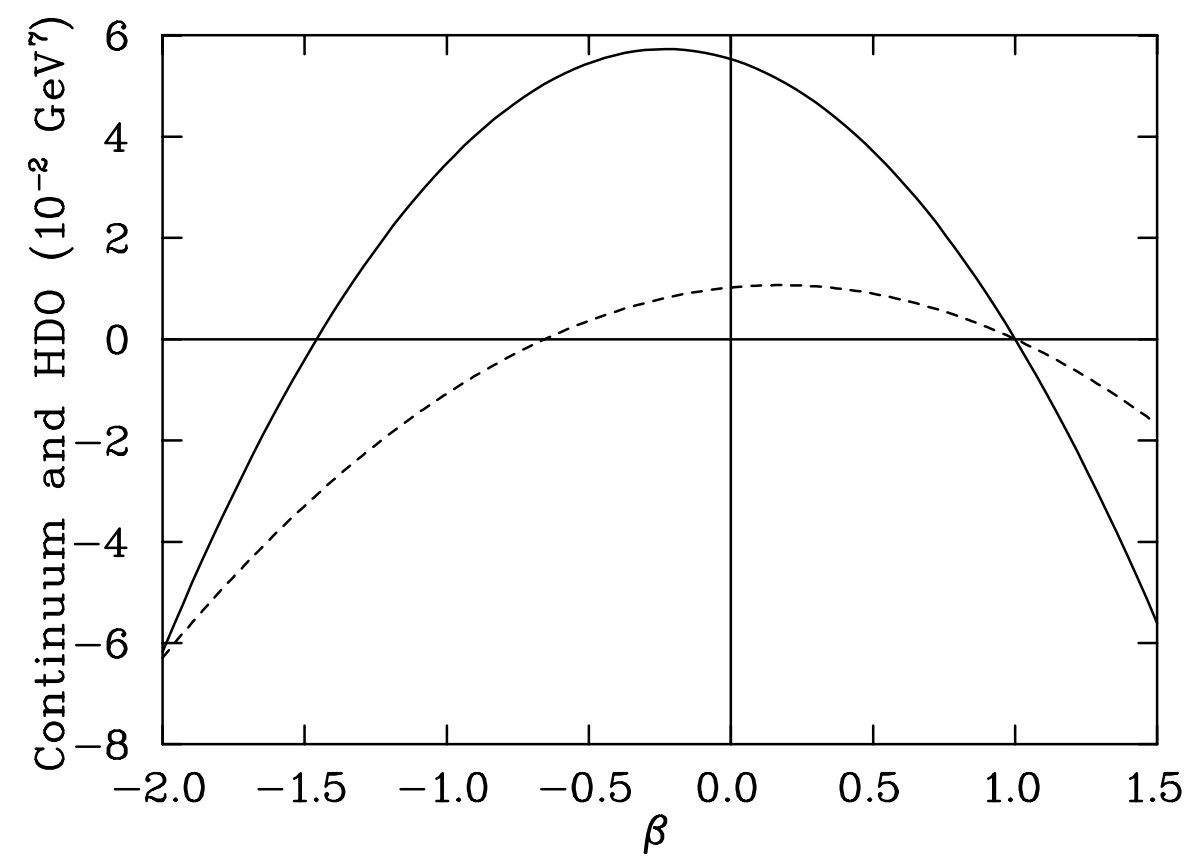

FIG. 10. Continuum model (solid curve) and HDO (dashed curve) contributions to the Borel improved QCD-SR of (6.1b) plotted as a function of the interpolating field mixing parameter $\beta$.

nucleon for small Borel masses.

Hence, errors made in truncating the OPE are dominated by errors in the $\chi_{1} \bar{\chi}_{1}$ component of the general correlator. The relative error in the OPE truncation can be reduced by adding $\chi_{2}$ components to the correlator. However, the $\chi_{2}$ components in the OPE are simply subtracted off again by the continuum model terms. Hence the relevant error is the absolute error. For $|\beta| \lesssim 1$, this error is dominated by $\chi_{1} \bar{\chi}_{1}$ components of the correlator. As a result, OPE truncation errors are approximately independent of $\beta$. This crucial point has been neglected in previous arguments regarding the optimal nucleon interpolating field.

Since the errors made in truncating the OPE are dominated by errors in the $\chi_{1} \bar{\chi}_{1}$ component of the general correlator the criteria of a $10 \%$ limit on the HDO contribution to the OPE should be applied to sum rules when $\beta=0$. Once this lower limit is established, it may be used for all $|\beta| \lesssim 1$. We will refer to this approach for determining the valid Borel regime as the "refined" method in the following.

The optimal nucleon interpolator must involve $\chi_{1}$, as this interpolator is required to maintain overlap with the ground state. The task is to determine the optimal mixing of $\chi_{2}$. Since $\chi_{2}$ has negligible overlap with the nucleon, the ground state contribution to a sum rule is also independent of $\beta$. Hence, the size of the continuum model contribution is the predominant factor in determining the optimal interpolator. Figure 10 illustrates the contributions of the continuum model terms in $(6.1 \mathrm{~b})$ for $M=0.94 \mathrm{GeV}$ and $w_{2}=1.4$ $\mathrm{GeV}$. The following discussion is not dependent on the precise values of these parameters. The first point to be made is that contributions from the continuum model are largest for $\beta \sim-0.2$. This selection of mixing is the worst possible choice for extracting information on the ground state nucleon. 
Figure 10 also indicates it is possible to have vanishing continuum model contributions at $\beta \simeq-1.5$ or $\beta=1$. However, we are relying on the continuum model to account for strength in the correlator that does not have its origin in the ground state. Without a continuum model, one would need to include additional poles on the right-hand-side of (6.1b) to account for positive and negative parity excitation strength. For $\beta<-1.5$ or $\beta>1.0$ the correlator is negative indicating the sum rule is saturated by a negative parity state.

Thus the optimal interpolator is $\beta \sim-1.2$ or $\beta \sim 0.8$. To discriminate between these two regimes, we turn to the higher-dimension-operator contributions which do not contribute to the continuum model. It is these terms that provide crucial information on whether the strength in the correlator lies in the ground state or the excited states. If these terms are absent, the optimal fit of the correlator is obtained when $\tilde{\lambda}_{\mathcal{O}} \rightarrow 0$ and $w_{2} \rightarrow 0$. In this case the continuum model and the OPE become equivalent via the Laplace transform of (2.26) and the fit is perfect. Hence the higher-dimension-operator contributions should be large in magnitude. A change in sign from the leading terms of the OPE will also assist in distinguishing ground state strength from excited state strength as the change in the curvature of the correlator will be more prominent.

The last term of $(6.1 \mathrm{~b})$ is independent of the continuum model, and its value is plotted as a function of beta in Figure 10. The contributions are larger for $\beta \sim-1.2$ than for $\beta \sim+0.8$. In addition, the sign of the contribution is opposite that of the continuum model contributions. Hence the preferred regime is $\beta \sim-1.2$. These arguments are supported by the numerical analysis described below.

In summary, the lattice results indicate OPE convergence, and ground state pole contributions are approximately independent of $\beta$. Considerations of the size of continuum model contributions and the sign and magnitude of operators independent of the continuum model leads to the preferred value of

$$
\beta=-1.2 \pm 0.1
$$

A more precise determination of $\beta$ will depend on the details of limits for continuum model and HDO contributions, condensate values, and other parameters of the sum rules.

At $\beta=-0.2$, where the leading term of the OPE is stationary with respect to $\beta$ 10,58, the continuum contributions are maximal. The positive value and small magnitude of the HDO contribution indicates the stability of the leading terms of the OPE will not be realized as stability in the ground state mass, coupling, nor in the continuum threshold.

Another point of view argues that the optimal interpolating field is one that eliminates direct instanton contributions from the correlator [55]. However, such arguments are based on the assumption that direct instantons necessarily contribute to the nucleon correlator in a significant manner. Section $\mathbb{\mathbb { X }}$ will demonstrate that direct instantons are not necessary to maintain sum rule self consistency for a wide range of interpolating fields. The $\beta$ dependence of the direct instanton contributions to $(6.1 \mathrm{~b})$ is proportional to $13 \beta^{2}+10 \beta+13 \neq 0$. Minimization of instanton contributions would place $\beta=-5 / 13$, very near $\beta=-0.6$ where the HDO term of (6.1b) vanishes and where continuum contributions are near their peak. Isolation of ground state contributions is nearly impossible in this regime. Therefore minimization of direct instanton contributions does not lead to an optimal interpolating field.

While it is important to establish the optimal mixing of interpolating fields for QCD-SR analyses, one should not overlook the fact that there is a range of values for $\beta$ where the 
sum rules are expected to work. Moreover, the ground state contribution to all these sum rules is equivalent to the $1 \%$ level [13]. In other words, the right-hand side of (6.1b) for a single pole plus continuum model is independent of $\beta$. After the first sum rule is written down, additional sum rules may be introduced with merely one new fit parameter (the continuum threshold) per sum rule. Since direct instanton contributions to the sum rules are not independent of $\beta$ [60], one has an excellent opportunity to see if direct instanton contributions really are necessary to maintain sum-rule consistency. This issue is addressed in Section [X] below.

\section{D. "Ioffe Formula"}

Having discovered that $\chi_{2}$ has negligible overlap with the nucleon ground state, it might be interesting to return to the "Ioffe Formula" this time generalized for arbitrary $\beta$

$$
M_{N} \simeq-\left[\frac{7-2 \beta-5 \beta^{2}}{5+2 \beta+5 \beta^{2}}\right] \frac{4(2 \pi)^{2}\langle\bar{q} q\rangle}{M^{2}} .
$$

For $\beta=0, M_{N}=2.5 \mathrm{GeV}$ when $M=1 \mathrm{GeV}$, or $M_{N}=1.4 \mathrm{GeV}$ when $M=M_{N} \mathrm{GeV}$. The explicit dependence on the choice of interpolating field and the Borel mass limits the significance of the "Ioffe formula" to a qualitative role at best.

\section{E. Nucleon Sum Rule at 1 for $\beta=-1.2$}

Here we focus on the more reliable sum rule of $(6.1 \mathrm{~b})$ at the optimal interpolator mixing of $\beta=-1.2$. Fortunately, the $\alpha_{s}$ corrections for the leading order operator of the OPE are much smaller than for the identity operator at the order of $10 \%$. The $\beta$-dependent coefficient of the quark condensate in (6.1b) becomes [54]

$$
7\left(1+\frac{15}{14} \frac{\alpha_{s}}{\pi}\right)-2 \beta\left(1+\frac{3}{2} \frac{\alpha_{s}}{\pi}\right)-5 \beta^{2}\left(1+\frac{7}{10} \frac{\alpha_{s}}{\pi}\right)
$$

These corrections are small relative to uncertainties in the other terms of the correlator and can be absorbed by a small shift in the condensate itself. Since our QCD parameter set is extracted from analyses with neglected $\alpha_{s}$ corrections, we shall do the same here.

Armed with knowledge of the optimal interpolator, and a refined understanding of OPE convergence tests, we now present the QCD-SR predictions for the nucleon spectral properties from spin-1/2 interpolators. Figure 11 displays the valid Borel regime for this sum rule. The HDO contributions are less than $10 \%$ of the OPE contributions for this fit when $\beta=0$, as indicated by the solid curve. As described above, contributions from correlator components known to be more convergent increase the HDO operator contributions making resolution of the pole from the continuum more probable.

Figure 12 displays the fit for this sum rule. The near perfect linearity of the OPE-continuum side of $\Pi_{S}(M)$ supports the arguments presented above. Despite $40 \%$ contributions of the HDO term to this sum rule, the OPE-continuum side displays the 


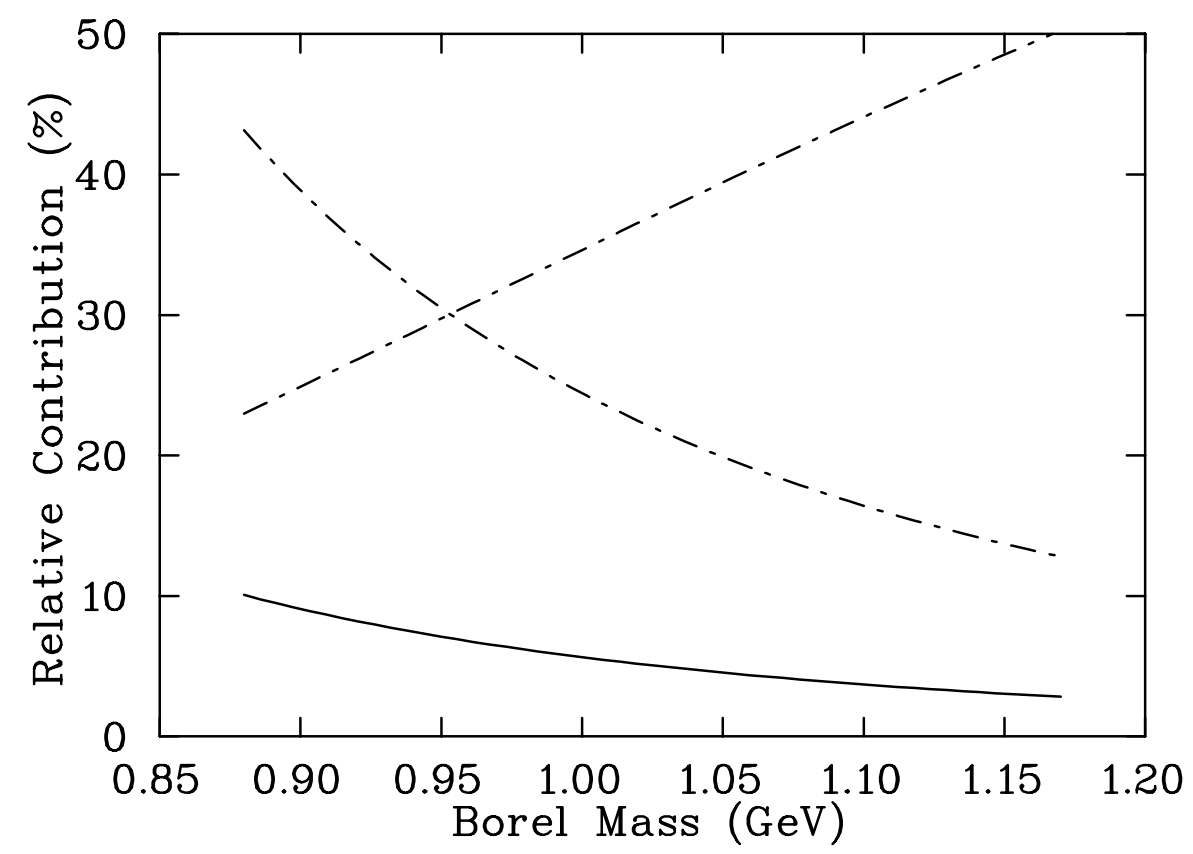

FIG. 11. The valid Borel regime for the sum rule of (6.1b) at the optimal $\beta=-1.2$. Relative HDO and continuum model contributions are illustrated (dot-dash curves). The HDO contributions are less than $10 \%$ of the OPE contributions for this fit when $\beta=0$, as indicated by the solid curve.

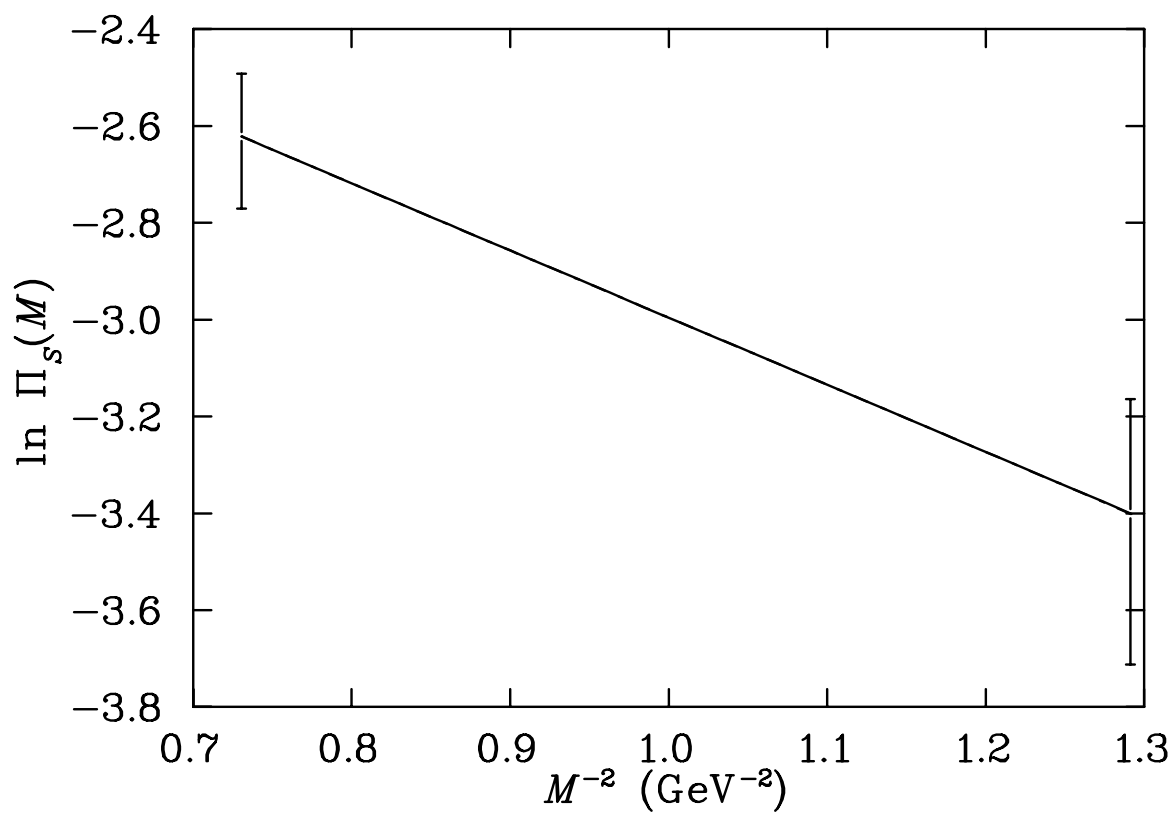

FIG. 12. Three parameter fit of (6.1a) for the residue $\lambda_{\mathcal{O}}$, the continuum model threshold $w_{2}$, and the nucleon mass. The near perfect linearity of the OPE-continuum side (hidden dot-dash line) of $\Pi_{S}(M)$ supports the arguments surrounding the refined determination of the valid Borel regime . 
anticipated behavior of reasonable OPE convergence. The optimal fit parameters obtained from consideration of 1000 QCD parameter sets are

$$
\tilde{\lambda}_{\mathcal{O}}^{2}=0.20 \pm 0.12 \mathrm{GeV}^{6}, \quad w_{2}=1.53 \pm 0.41 \mathrm{GeV}, \quad M_{N}=1.17 \pm 0.26 \mathrm{GeV}
$$

The uncertainty of the nucleon mass is more than double the traditional expectation at 260 MeV. Figures 13, 14, and 15 display histograms for the nucleon mass, threshold and residue respectively. These histograms will aid in the discussion of the following section.

Table 1 compares the prediction for $\lambda_{\mathcal{O}}$ with other estimates of $\lambda_{\mathrm{SR}}=2 \lambda_{\mathcal{O}}$. This residue corresponds to Ioffe's interpolator of (2.5) and is expected to be independent of $|\beta| \lesssim 1$ to the order of $1 \%$ [13].

In the past the community has emphasized the importance of the independence of the nucleon mass from the Borel mass parameter. It is common to find a plot of the nucleon mass extracted from the ratio method discussed above as a function of the Borel mass. While independence of the spectral parameters from the Borel mass is certainly desirable, the ratio method is potentially problematic in practice. We have already seen that the reliabilities and validities of two sum rules are usually different and could even result in separate valid regimes. This feature cannot be revealed in the ratio of the two sum rules. In addition, one can always achieve flatness in the ratio method in the large Borel mass region, where both sides of the sum rules are dominated by the continuum model. However, one learns little about the lowest pole in this case.

Contact with the plateau criterion may be made by rewriting the sum rule of interest solving for the spectral parameter in question. For example, one can express the nucleon mass in the sum rule of ( $6.1 \mathrm{~b})$ as,

$$
M_{N}=M\left[\ln \left(\frac{\tilde{\lambda}_{\mathcal{O}}^{2}}{\Pi_{S}(M)}\right)\right]^{1 / 2},
$$

where $\Pi_{S}(M)$ denotes the left-hand side of (6.1b). Indeed a plot of $M_{N}$ versus the Borel parameter $M$ is very flat within the valid region. This information is already apparent

TABLE I. Comparison of predictions for $\lambda_{\mathrm{SR}}=2 \lambda_{\mathcal{O}}$ for various approaches to QCD.

\begin{tabular}{llc}
\hline \hline Approach & Reference & $\begin{array}{c}\lambda_{\mathrm{SR}} \\
\left(\times 10^{-2} \mathrm{GeV}^{3}\right)\end{array}$ \\
\hline QCD Sum Rule & This work & $2.2 \pm 0.7$ \\
Lattice (mean-field improved) & Leinweber [13] & $2.7 \pm 0.5$ \\
Lattice (conventional renormalization) & Gavela et al. [61] & 2.4 \\
Lattice (coordinate space) & Chu et al. [62] & $2.2 \pm 0.4$ \\
Instanton Liquid & Schafer et al. [63] & $3.2 \pm 0.1$ \\
Baryon wave functions $\left(x^{2} \rightarrow 0\right)$ & Brodsky et al. [64] & 12 \\
Quark Model & Thomas and McKellar [65] & 8 \\
Bethe-Salpeter amplitude & Tomozawa [66] & 2.5 \\
Quark Model & Milosevic et al. [67] & 2 \\
MIT Bag Model & Donoghue and Golowich [68] & 1.27 \\
\hline \hline
\end{tabular}




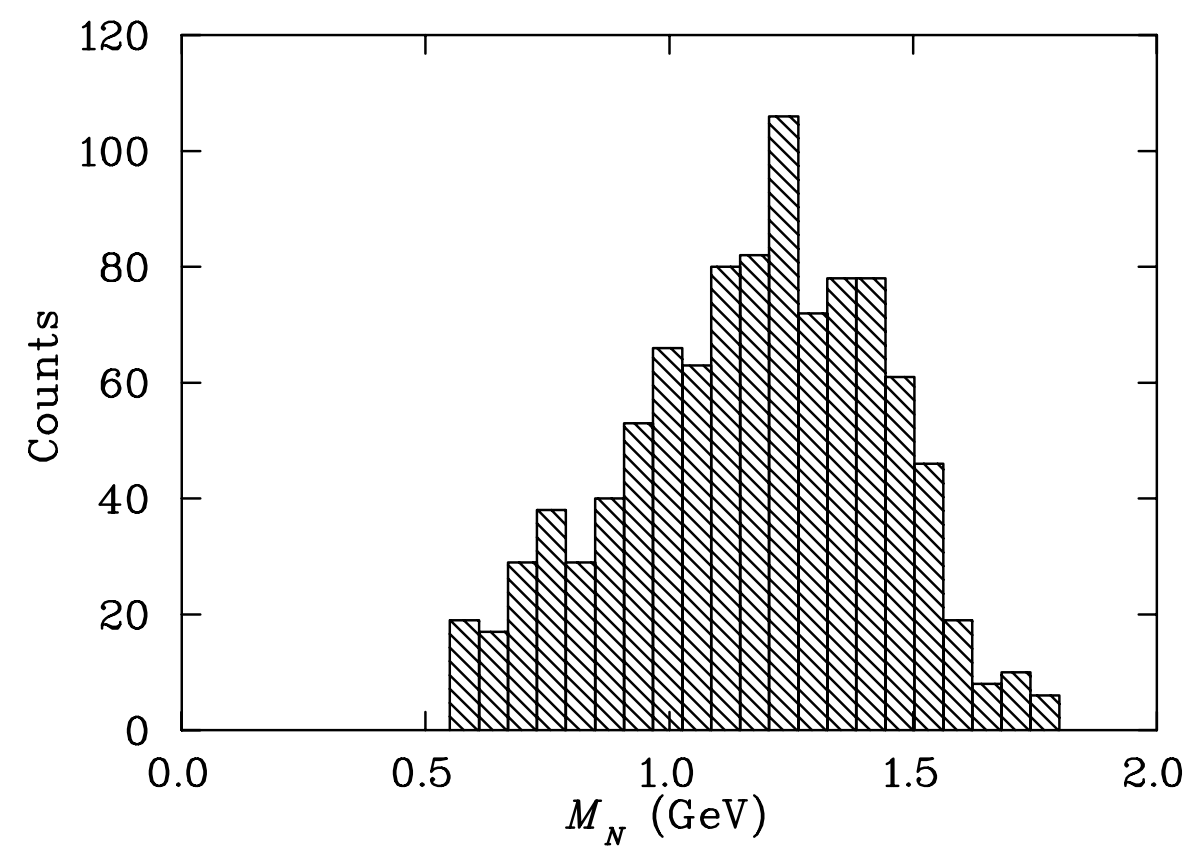

FIG. 13. Histogram for the nucleon mass obtained from fits of $(6.1 \mathrm{~b})$ at the optimal $\beta=-1.2$ for $1000 \mathrm{QCD}$ parameter sets.

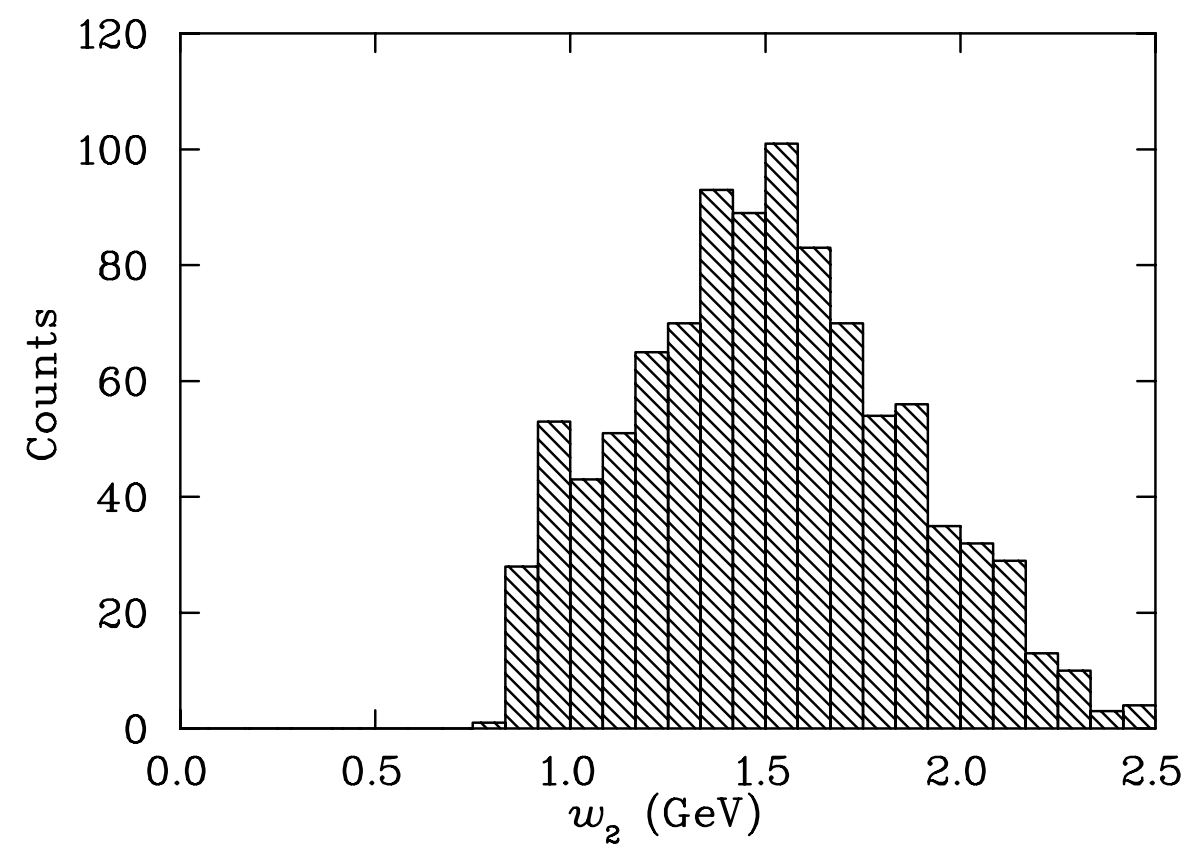

FIG. 14. Histogram for the continuum threshold obtained from fits of $(6.1 \mathrm{~b})$ at the optimal $\beta=-1.2$ for $1000 \mathrm{QCD}$ parameter sets. 


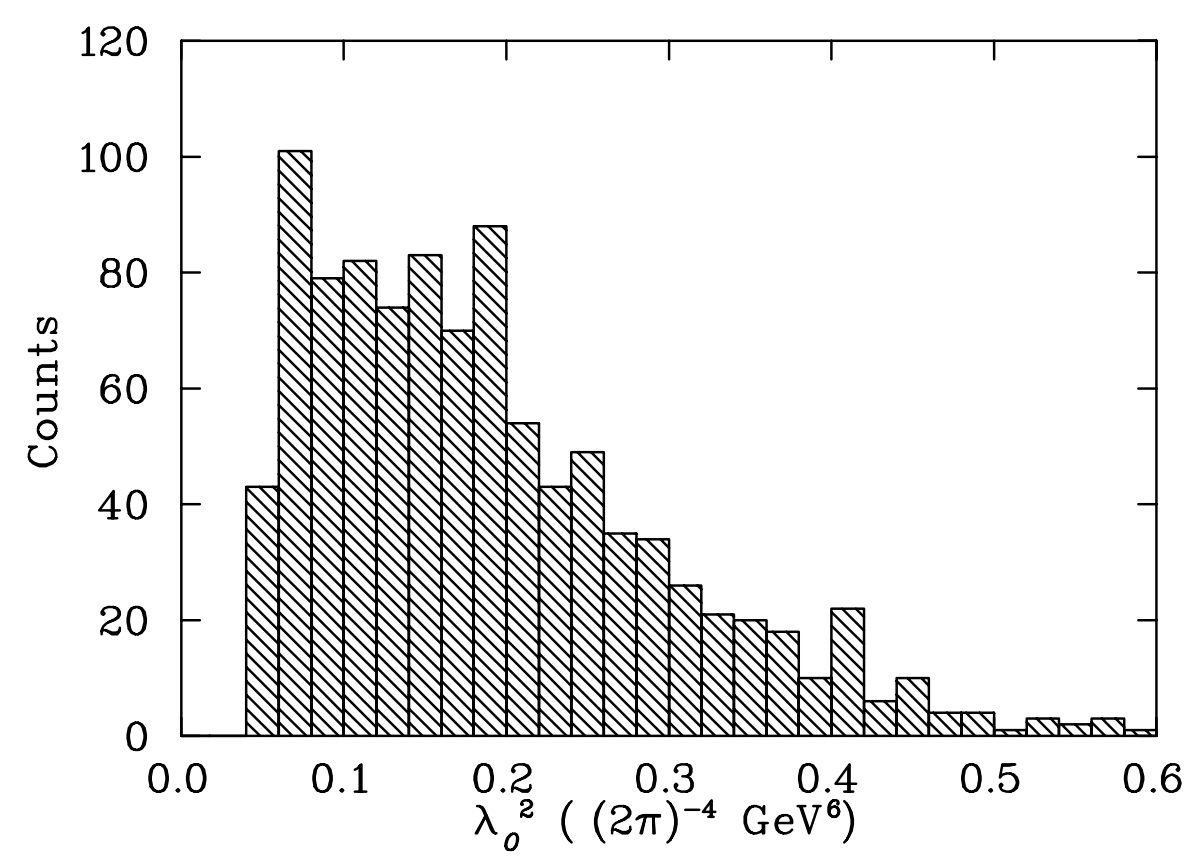

FIG. 15. Histogram for the residue of the nucleon pole obtained from fits of $(6.1 \mathrm{~b})$ at the optimal $\beta=-1.2$ for 1000 QCD parameter sets.

in figure 12 where the near perfect linearity of the OPE-continuum side of the sum rule indicates independence of the fitted mass from the Borel parameter. For a similar analysis of the $\rho$-meson in finite density nuclear matter see [6].

Returning to the sum rule at the structure $\gamma \cdot p$ of $(6.1 \mathrm{a})$, one finds the valid Borel regime disappears when the refined OPE convergence criteria is applied. The dashed curves of figure 16 display the naive Borel regime for (6.19) at $\beta=-1$ used in the fit of (6.4). The solid curve illustrates the HDO contributions to the slowest converging component of the correlator $(\beta=0)$. This curve fails to drop below $10 \%$ before the continuum model contributions exceed $50 \%$. The HDO of the faster converging components reduces the HDO contribution of the slowest converging component to give the illusion of reasonable OPE convergence at $\beta=-1$. This is most likely the explanation for the failure of the sum rule of (6.1a); the absence of a valid Borel regime.

\section{F. Nucleon Sum Rule at 1 for $\beta=+0.8$}

Having considered the most favorable interpolator for the nucleon, let us now examine the QCD-SR predictions for a less than favorable interpolator. At $\beta=+0.8$, figure 10 indicates the continuum model contributions can be reasonably small. Hence it should be possible to extract ground state information that is reliable. However, the HDO term is of the same sign and small relative to the leading order contributions. The difficulty here will be resolving the ground-state pole from the approximate continuum model.

The lower limit of the valid Borel regime is determined by the OPE contributions which are independent of the phenomenological fit parameters and independent of $\beta$. Hence this 


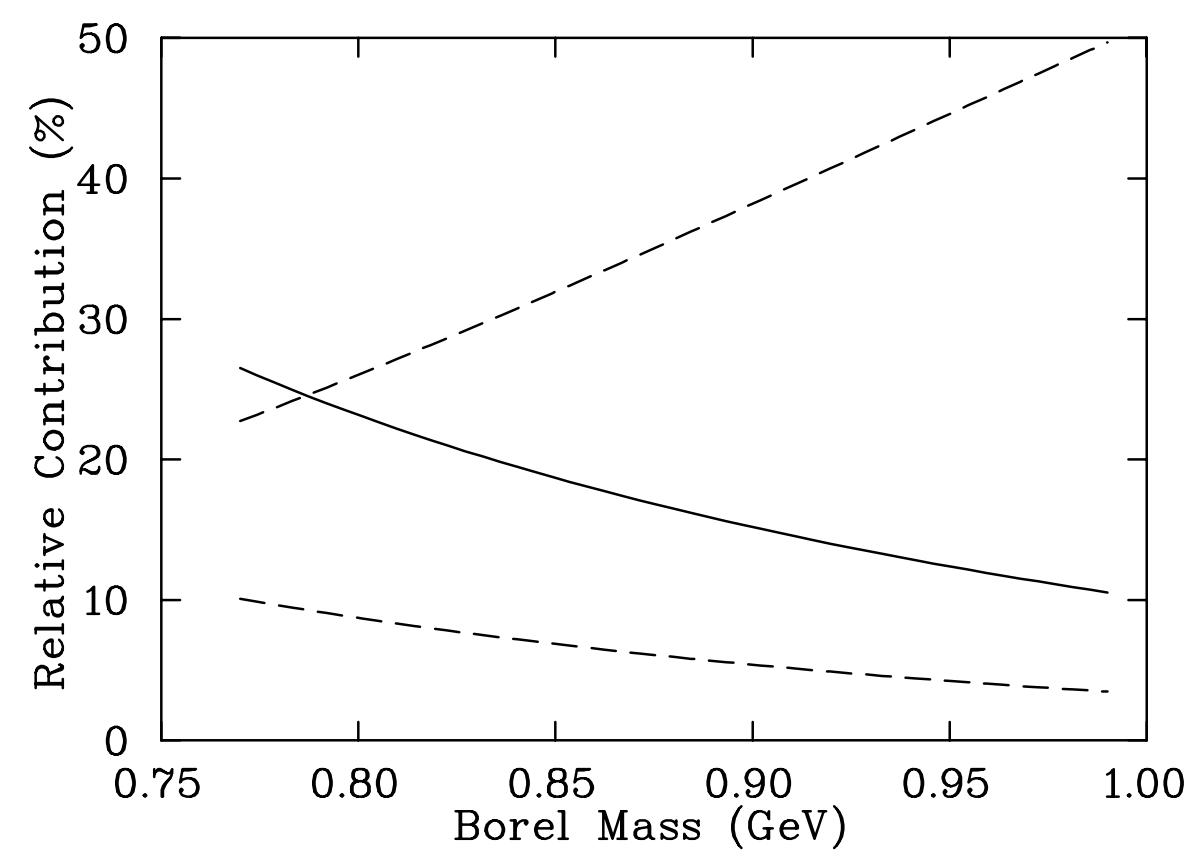

FIG. 16. The naive Borel regime for (6.1a) at $\beta=-1$. The solid curve illustrates the HDO contributions to the slowest converging component of the correlator $(\beta=0)$. The failure of this curve to drop below $10 \%$ before the continuum model contributions exceed $50 \%$ indicates the absence of a valid Borel regime.

limit is known to be $0.88 \mathrm{GeV}$. The inherent instability of this sum rule makes it difficult to determine the upper limit. Figure 10 suggests that continuum contributions should be similar to that for $\beta=-1.2$ and therefore we take an upper limit of $1.17 \mathrm{GeV}$, as in Figure 11.

Figures 17, 18, and 19 display histograms for the nucleon mass, threshold and residue obtained from (6.1b) for $\beta=+0.8$. Figure 17 resolves two regimes for the nucleon mass and figure 18 suggests the presence of at least three regimes.

In section $\nabla \Pi 1$ the nature of these three regimes are resolved via a correlation analysis. As mentioned earlier there are two ways to fit a sum rule. If the terms which do not contribute to the continuum model are insufficient to resolve the pole, a good fit may be obtained by letting the continuum threshold and the residue of the pole go to zero. One then has an approximate Laplace transform relating the two sides of the sum rule. These fits are represented by the left-most shoulder of the histograms.

An intermediate regime arises when the sum rules are capable of resolving a threshold at which strength in the spectral density begins. However, a gap between ground and excited states is not resolved. The peak at $0.5 \mathrm{GeV}$ in the continuum threshold is representative of these fits. For these fits the nucleon mass is scattered about typical values but with with a small residue. The smaller residue compensates for strength from the continuum model.

Finally, the third regime represented by the broad right-most peak in the continuum threshold histogram corresponds to more standard fits where a gap between the ground and first excited state has been resolved. 


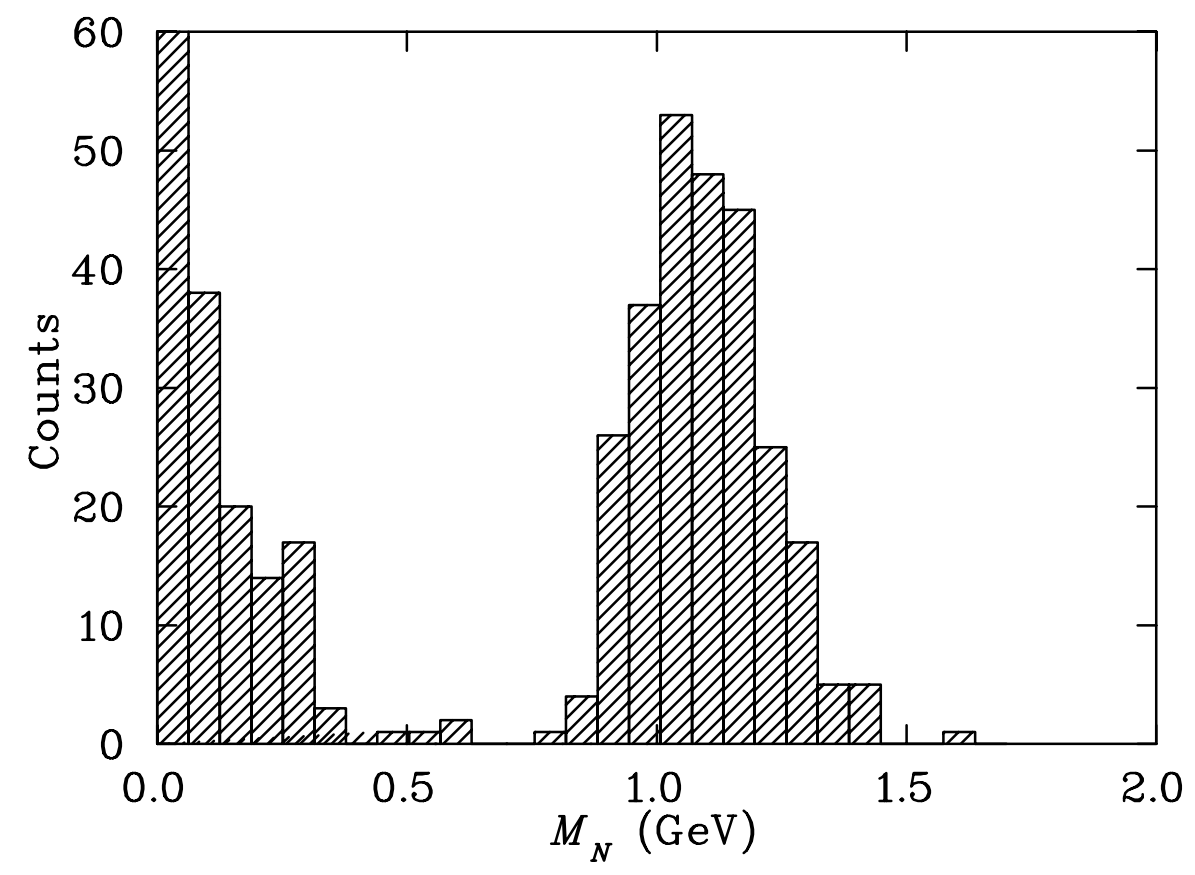

FIG. 17. Histogram for the nucleon mass obtained from fits of (6.1b) at $\beta=+0.8$ for 1000 QCD parameter sets. When the pole is resolved from the continuum, the histogram compares favorably with that of figure 13.

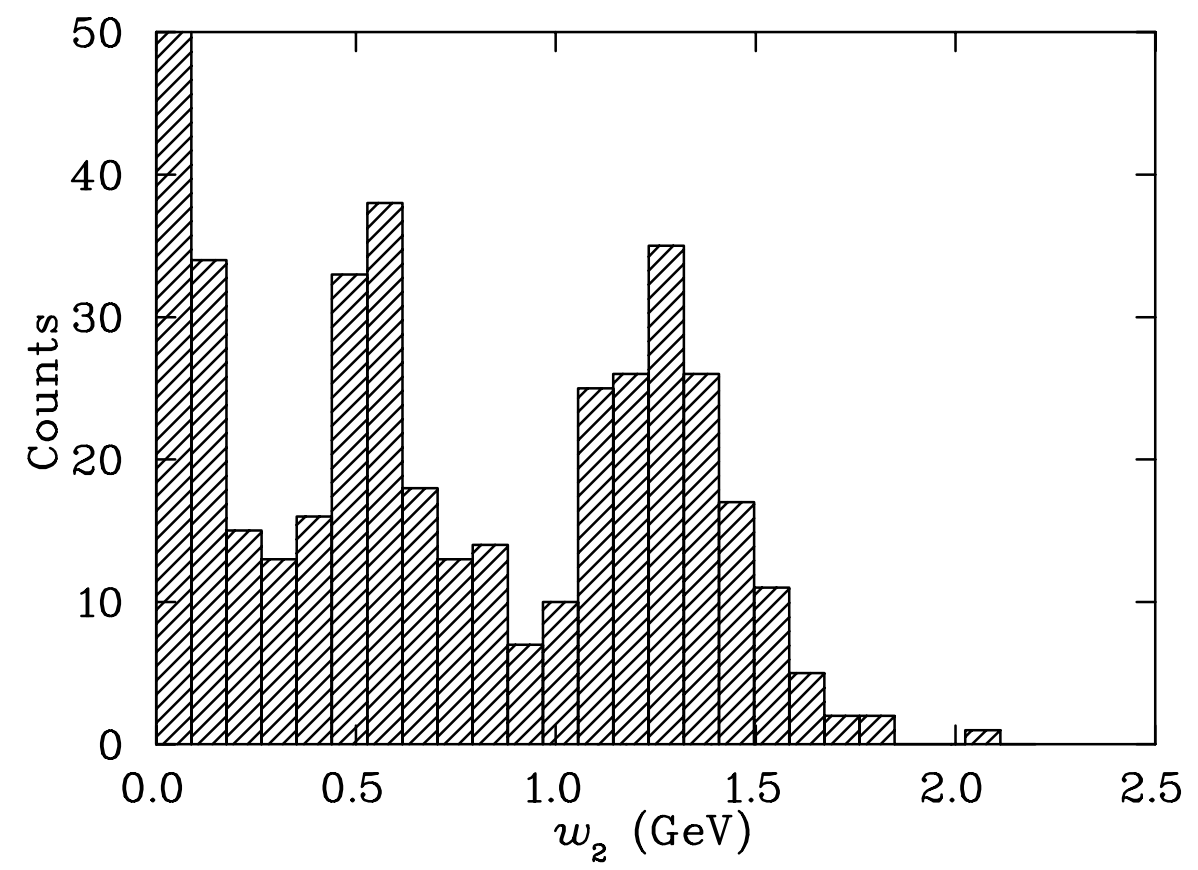

FIG. 18. Histogram for the continuum threshold obtained from fits of (6.1b) at $\beta=+0.8$ for 1000 QCD parameter sets. The three types of sum rule fits are clearly resolved. 


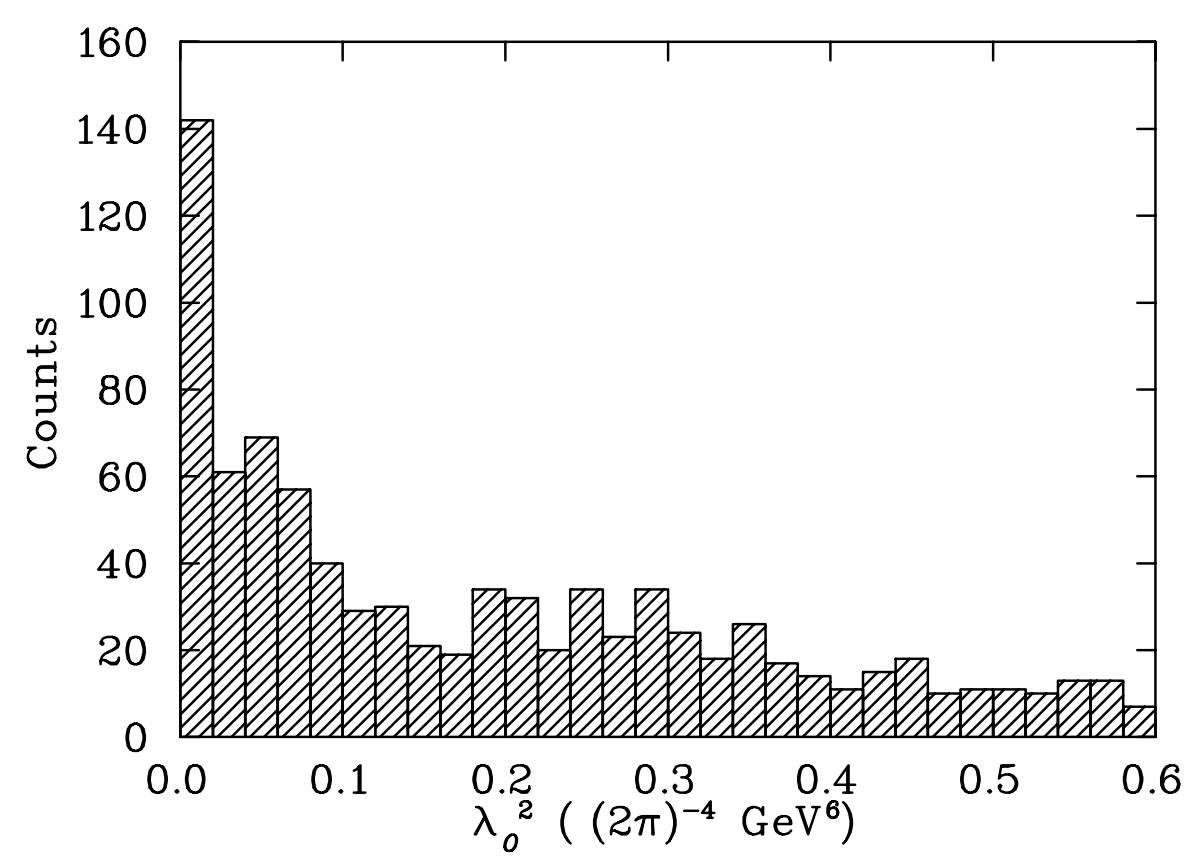

FIG. 19. Histogram for the residue of the nucleon pole obtained from fits of $(6.1 \mathrm{~b})$ at $\beta=+0.8$ for 1000 QCD parameter sets. Additional counts at small $\lambda_{\mathcal{O}}^{2}$ allows for additional strength from the continuum model.

It is encouraging to see the similarity in the nucleon mass distributions of figures 17 and 13 when the sum rules resolve a pole plus continuum fit. This agreement comes about without the need for direct instanton contributions.

\section{NUCLEON SUM RULES: SPIN-1/2 AND 3/2 INTERPOLATORS}

\section{A. Sum Rule Derivation}

The spin-3/2 interpolating field for the nucleon is [14,27]

$$
\chi_{3 / 2}^{\mu}(x)=\epsilon^{a b c}\left[\left(u^{a \mathrm{~T}}(x) C \sigma_{\rho \lambda} d^{b}(x)\right) \sigma^{\rho \lambda} \gamma^{\mu} u^{c}(x)-\left(u^{a \mathrm{~T}}(x) C \sigma_{\rho \lambda} u^{b}(x)\right) \sigma^{\rho \lambda} \gamma^{\mu} d^{c}(x)\right] .
$$

Nucleon to vacuum matrix elements of $\chi_{3 / 2}^{\mu}$ are defined as

$$
\begin{aligned}
& \left\langle 0\left|\chi_{3 / 2}^{\mu}\right| 1 / 2^{+}\right\rangle=\left(\alpha p^{\mu}+\lambda_{3 / 2} \gamma^{\mu}\right) \gamma_{5} u(p), \\
& \left\langle 0\left|\chi_{3 / 2}^{\mu}\right| 1 / 2^{-}\right\rangle=\left(\alpha p^{\mu}+\lambda_{3 / 2} \gamma^{\mu}\right) u(p)
\end{aligned}
$$

The appearance of $\gamma_{5}$ on the right-hand side matches the parity of $\chi_{3 / 2}^{\mu}$. The property $\gamma_{\mu} \chi_{3 / 2}^{\mu}=0$ provides the relation

$$
\alpha=\frac{4 \lambda_{3 / 2}}{ \pm M_{N}}
$$


In an analogous manner we work with the generalized spin- $1 / 2$ interpolator

$$
\chi_{\mathcal{O}}^{\mu}=\gamma^{\mu} \gamma_{5} \chi_{\mathcal{O}},
$$

which has the matrix elements of (7.2) with $\alpha=0$ and $\lambda_{3 / 2} \rightarrow \lambda_{\mathcal{O}}$. Overlap of the spin$3 / 2$ interpolating field with the generalized spin- $1 / 2$ interpolator of (2.3) gives rise to four Dirac- $\gamma$ structures

$$
\left\langle 0\left|\chi_{\mathcal{O}}^{\mu}\right| 1 / 2^{ \pm}\right\rangle\left\langle 1 / 2^{ \pm}\left|\bar{\chi}_{3 / 2}^{\nu}\right| 0\right\rangle=\lambda_{\mathcal{O}} \lambda_{3 / 2}\left\{-\gamma^{\mu} \gamma^{\nu}\left(\gamma \cdot p \pm M_{N}\right) \pm \frac{4}{M_{N}} \gamma^{\mu} p^{\nu} \gamma \cdot p-2 \gamma^{\mu} p^{\nu}\right\},
$$

from which three independent sum rules are obtained.

$$
\begin{gathered}
\left(\gamma_{\mu} \gamma_{\nu} \gamma \cdot p\right): \quad \kappa_{N} a^{2} L^{20 / 27}-\frac{9-\beta}{24} \frac{m_{0}^{2} a^{2}}{M^{2}} L^{2 / 9} \\
=\tilde{\lambda}_{\mathcal{O}} \tilde{\lambda}_{3 / 2} e^{-M_{N}^{2} / M^{2}} \\
\left(\gamma_{\mu} \gamma_{\nu}\right): \quad \frac{1}{2} a M^{4} L^{8 / 27}\left[1-e^{-w_{3}^{2} / M^{2}}\left(\frac{w_{3}^{2}}{M^{2}}+1\right)\right]+\frac{1+3 \beta}{96} a b L^{8 / 27} \\
=\tilde{\lambda}_{\mathcal{O}} \widetilde{\lambda}_{3 / 2} M_{N} e^{-M_{N}^{2} / M^{2}}, \\
\left(\gamma_{\mu} p_{\nu} \gamma \cdot p\right): \quad \frac{1}{2} a M^{2} L^{8 / 27}\left[1-e^{-w_{3}^{2} / M^{2}}\right]-\frac{3-\beta}{16} m_{0}^{2} a L^{-2 / 9} \\
-\frac{1+3 \beta}{96} \frac{a b}{M^{2}} L^{8 / 27} \\
=\frac{\widetilde{\lambda}_{\mathcal{O}} \widetilde{\lambda}_{3 / 2}}{M_{N}} e^{-M_{N}^{2} / M^{2}} .
\end{gathered}
$$

The corresponding Dirac- $\gamma$ structure from which the sum rule is obtained is indicated on the left. The sum rule at the structure $\gamma_{\mu} p_{\nu}$ is identical to that for $\gamma_{\mu} \gamma_{\nu} \gamma \cdot p$.

The leading term of the sum rule at the structure $\gamma_{\mu} \gamma_{\nu} \gamma \cdot p$ is the four-quark condensate. As such the OPE of this sum rule is poorly known. In addition, there are no terms from which a continuum model may be constructed. For an analysis and discussion of this sum rule under the assumption of factorization see [9].

The sum rule at the structure $\gamma_{\mu} q_{\nu} \gamma \cdot p$ is a very favorable sum rule. The dimensionality of the structure at which the sum rule is extracted allows the HDO terms of the sum rule to approach the factorial suppression regime of the Borel transform thus improving the truncated OPE convergence. Moreover, there are two terms which are not used in the continuum model. Hence there should be better resolution of the pole from the continuum.

The mixed condensate is absent in (7.6b) for all $\beta$. With only two pieces of information on the OPE side of the sum rule, this sum rule is of little use on it's own. Fortunately, both (7.6b) and (7.69) are extracted from Dirac $\gamma$-structures in which even- and odd-parity excitations contribute with opposite signs. Hence it is reasonable to set the continuum model thresholds equal for these two sum rules and this provides greater stability in the fit parameters. 
Since the terms of (7.6) used in the continuum model are independent of the interpolator mixing parameter $\beta$, the obvious selection for the optimal mixing is $\beta=0$. Of course, if the interpolator $\chi_{2}$ of (2.4b) doesn't contribute to the continuum model, nor to the ground state, it will be important to demonstrate that different values of $\beta$ lead to similar results. The expectation is that the continuum threshold will be dependent on $\beta$ while the mass and residue remain unchanged for $|\beta| \lesssim 1$. This dependence is examined in Section IX examining the necessity of direct instanton contributions.

Ioffe's selection of $\beta=-1$ as the optimal spin-1/2 interpolator is reasonably close to the determination of $\beta=-1.2$ in Section $\nabla I C$ for spin- $1 / 2$ to spin- $1 / 2$ interpolators. However, the optimal mixing is specific to each individual sum rule. Here we have found an optimal value quite different from Ioffe's preference and, as we shall see, Ioffe's selection of $\beta \sim-1$ fails to provide a sum rule with a valid regime.

\section{B. Sum Rule Analysis}

We begin by considering a simultaneous analysis of (6.1b) at $\beta=-1.2$, and $(7.6 \mathrm{~b}$ ) and (7.6c) at $\beta=0$. A valid Borel regime could not be found for $(6.1 \mathrm{~b})$ when analyzed in conjunction with the two additional sum rules of $(7.6 \mathrm{~b})$ and $(7.6 \mathrm{~d})$. Hence we will analyze $(7.6 \mathrm{~b})$ and $(\overline{7.6 \mathrm{~g}})$ alone. Figure 20 displays the valid Borel regimes for these two sum rules.

The corresponding three parameter fit of the residue $\widetilde{\lambda}_{\mathcal{O}} \widetilde{\lambda}_{3 / 2}$, threshold, $w_{3}$, and nucleon mass is indicated in figure 21. The sum rule of (6.1b) is also displayed for the Borel regime considered in Sec. VIE. The linearity of the OPE-continuum sides of the subtracted sum rules over a relatively broad Borel regime indicates the OPE is under reasonable control. However, since the continuum model dominates the phenomenology of (6.1b), we do not consider it further. The resultant fit parameters obtained from the consideration of 1000 QCD parameters sets are

$$
\begin{gathered}
M_{N}=0.96 \pm 0.08 \mathrm{GeV} \\
\tilde{\lambda}_{\mathcal{O}} \tilde{\lambda}_{3 / 2}=0.41 \pm 0.14 \mathrm{GeV}^{6}, \quad w_{3}=1.3 \pm 0.2 \mathrm{GeV}
\end{gathered}
$$

The uncertainty of $100 \mathrm{MeV}$ for the nucleon mass is a significant improvement over the traditional sum rule of (6.1b) alone as illustrated in figure 22. The value of the threshold lies roughly at the value suggested by the particle data tables of approximately $1.27 \mathrm{GeV}$; the Roper mass of $1.440 \mathrm{GeV}$ less the half width of $0.175 \mathrm{GeV}$. The value for $\lambda_{3 / 2}$ is a prediction that awaits vindication from alternative approaches.

The presence of a valid Borel regime common to both sum rules of (7.6b) and (7.60) provides an opportunity to apply the Monte-Carlo uncertainty analysis to the ratio method. The ratio of equations $(7.6 \mathrm{~b}) /(7.6 \mathrm{~g})$ provides a ground state contribution equal to $M_{N}^{2}$ and independent of the residues $\lambda_{\mathcal{O}} \lambda_{3 / 2}$. The optimal fit parameters are

$$
M_{N}=1.05 \pm 0.08 \mathrm{GeV}, \quad w_{3}=1.7 \pm 0.5 \mathrm{GeV}
$$

and agree with the previous results. The difference in the central values is due to the different Borel regimes used in the fit. Despite taking a ratio of sum rules, the uncertainty 


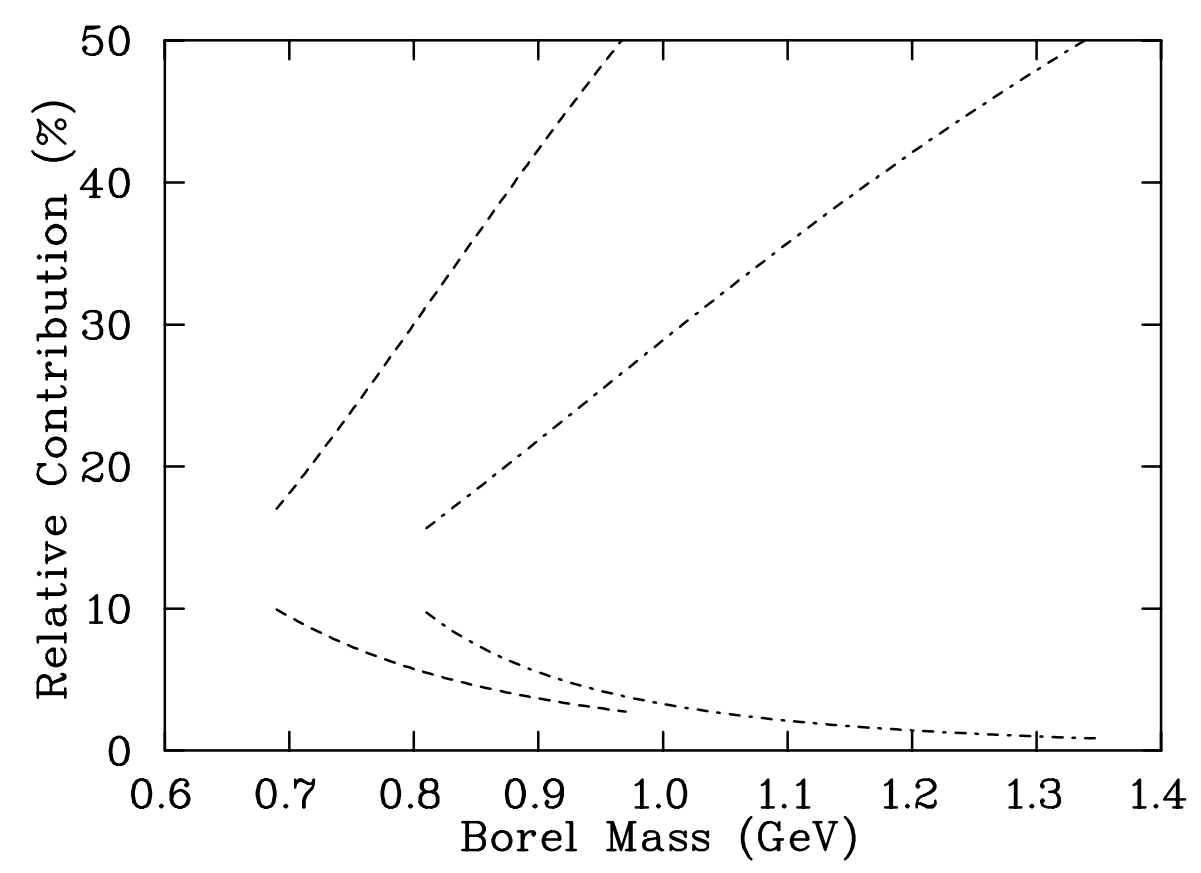

FIG. 20. The valid Borel windows for the nucleon sum rules of (7.6b) (dashed) and (7.60) (short dash-dot) at the optimal $\beta=0$. The relative HDO contributions limited to $10 \%$ and the continuum model contributions limited to $50 \%$ are illustrated.

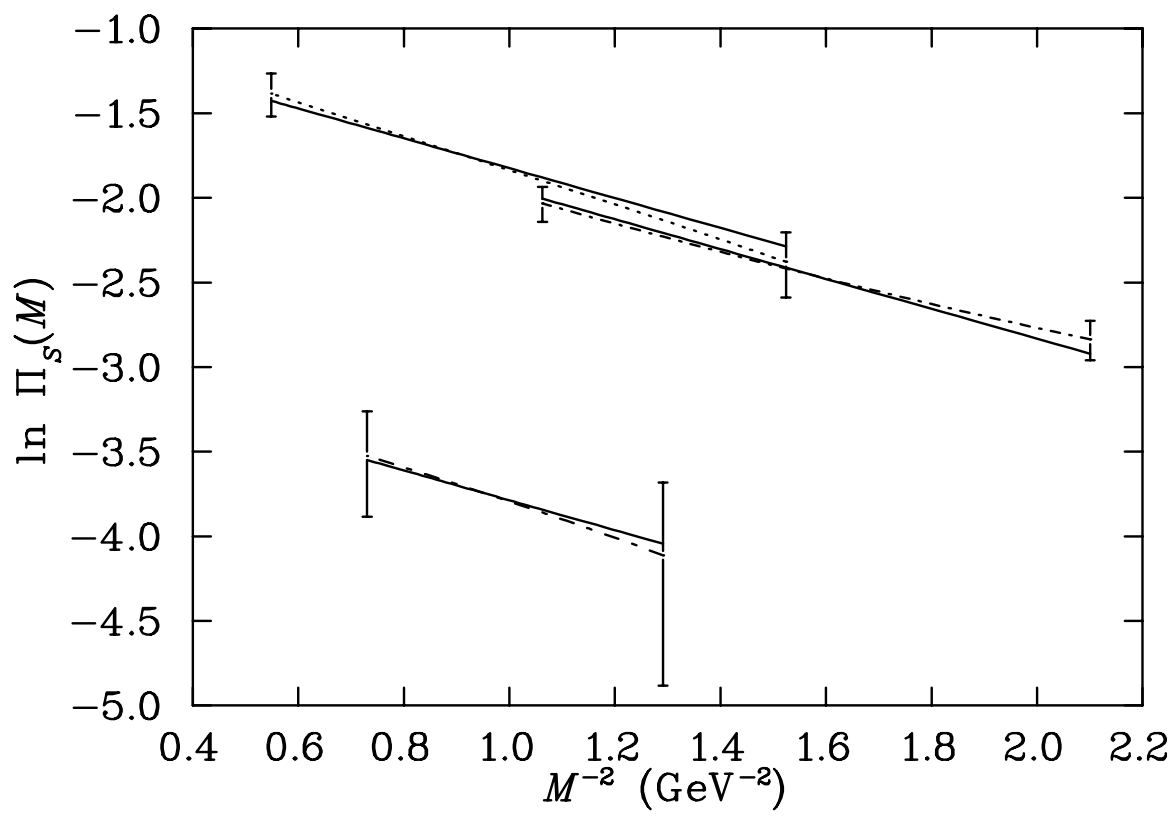

FIG. 21. A three parameter fit including $\lambda_{3 / 2}, M_{N}$ and $w_{3}$ of the nucleon sum rules of (7.6b) (dashed) and (7.60) (short dash-dot) at $\beta=0$. The sum rule of (6.1b) at $\beta=-1.2$ (long dash-dot) is also illustrated to demonstrate the consistency of all three sum rules. The solid lines illustrate the ground state contributions. 


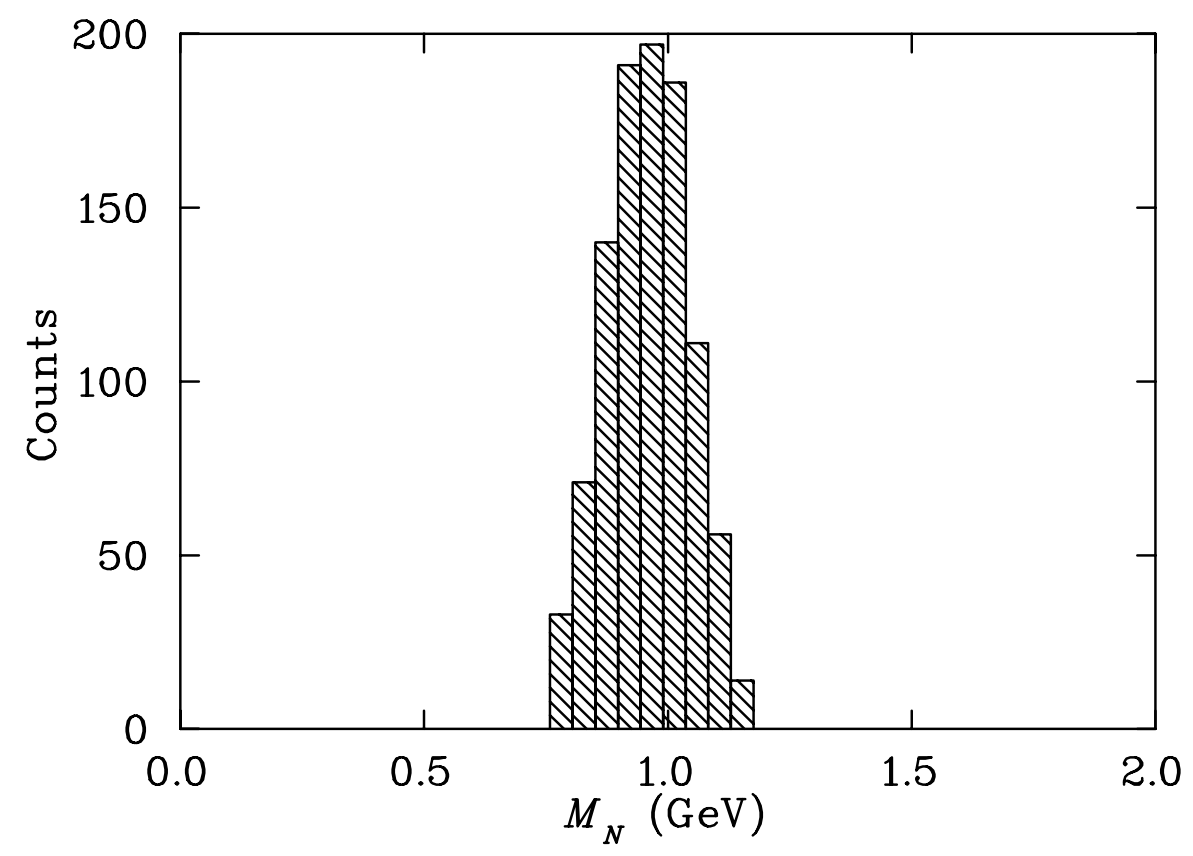

FIG. 22. Histogram for the nucleon mass obtained from fits of (7.6b) and (7.6c) at the optimal $\beta=0$ for 1000 QCD parameter sets.

in the nucleon mass remains nearly unchanged. The fit obtained from the full covariance matrix $\chi^{2}$ of (4.6) provides similar results and yields $\chi^{2} / N_{D F}=1.34$. This acceptable value suggests the distributions selected for the QCD parameters are in reasonable accord with the approximations inherent in the QCD-SR approach. Future studies might use the $\chi^{2} / N_{D F}$ to place restrictions on QCD parameter sets selected in the Monte-Carlo approach.

Table II summarizes an investigation of $\chi^{2} / N_{\mathrm{DF}}$ and uncertainties obtained from the analysis of ( $\overline{7.6 \mathrm{~b}})$ and $(\overline{7.6 \mathrm{c}})$ at $\beta=0$ for various relative errors of condensate input parameters. Results are based on samples of 200 condensate parameter sets. The ratio results are obtained from the ratio of these sum rules analyzed in the Borel regime common to both sum rules. A comparison of the uncertainties for the nucleon mass obtained from the ratio method and the standard analysis advocated here indicates there is very little if anything to be gained in the ratio approach to sum rule analysis.

Table 凹indicates a $\chi^{2} / N_{\mathrm{DF}} \simeq 1$ is obtained when the QCD input parameters are specified with $12 \%$ uncertainties. Hence, the best one can determine phenomenological parameters with the current implementation of QCD-SRs is 10\% accuracy for the nucleon mass and 25\% accuracy for the residue. This large uncertainty for the residue does not bode well for the analysis of three-point functions where the quantity of interest appears multiplied by the reside. The two-point function is used to normalize the three-point function, and it appears that this normalization is not well determined. Reduction of this uncertainty to the $10 \%$ level requires 5\% uncertainties on the QCD input parameters. However, the $\chi^{2} / N_{\mathrm{DF}}=18.7$ indicates this level of accuracy is beyond the current state of the art. Of course, this is not surprising since approximations at the level of $10 \%$ have been made in deriving the sum rules. 
TABLE II. An investigation of $\chi^{2} / N_{\mathrm{DF}}$ and uncertainties obtained from the analysis of (7.6b) and (7.60) at $\beta=0$ for various relative errors of condensate input parameters. Results are based on samples of 200 condensate parameter sets. Ratio results are obtained from the ratio of these sum rules analyzed in the Borel regime common to both sum rules. The last row summarizes the results for the condensate parameter set estimated in Sec. III.

\begin{tabular}{ccccccc}
\hline \hline Condensate & \multicolumn{3}{c}{ Sum Rule Ratio } & \multicolumn{3}{c}{ Standard Analysis } \\
Uncertainty & $\chi^{2} / N_{\mathrm{DF}}$ & $\begin{array}{c}M_{N} \\
(\mathrm{GeV})\end{array}$ & $\begin{array}{c}w_{3} \\
(\mathrm{GeV})\end{array}$ & $\begin{array}{c}M_{N} \\
(\mathrm{GeV})\end{array}$ & $\begin{array}{c}w_{3} \\
(\mathrm{GeV})\end{array}$ & $\begin{array}{c}\tilde{\lambda}_{\mathcal{O}} \tilde{\lambda}_{3 / 2} \\
\left(\mathrm{GeV}^{6}\right)\end{array}$ \\
\hline $20 \%$ & 0.11 & $1.04(12)$ & $1.78(91)$ & $0.96(11)$ & $1.29(26)$ & $0.40(15)$ \\
$15 \%$ & 0.26 & $1.04(10)$ & $1.73(75)$ & $0.96(10)$ & $1.29(22)$ & $0.40(12)$ \\
$13 \%$ & 0.69 & $1.04(9)$ & $1.72(68)$ & $0.95(9)$ & $1.28(19)$ & $0.40(10)$ \\
$12 \%$ & 1.13 & $1.04(8)$ & $1.70(65)$ & $0.95(8)$ & $1.27(17)$ & $0.39(9)$ \\
$10 \%$ & 2.22 & $1.04(6)$ & $1.67(47)$ & $0.95(7)$ & $1.26(14)$ & $0.39(8)$ \\
$5 \%$ & 18.74 & $1.02(3)$ & $1.47(6)$ & $0.95(3)$ & $1.26(7)$ & $0.38(4)$ \\
\hline Estimated & 1.34 & $1.05(8)$ & $1.72(52)$ & $0.96(8)$ & $1.28(20)$ & $0.41(14)$ \\
\hline \hline
\end{tabular}

\section{CORRELATIONS}

\section{A. $\chi^{2}$ Measures of Association}

To quantitatively examine the correlations among the QCD and phenomenological parameters, we utilize a contingency table analysis of two distributions [49] to determine the significance, and strength of correlations. For the correlations found to be significant, the linear correlation coefficient is calculated.

Determinations of the significance, probability and strength of correlations can be based on $\chi^{2}$ measures of association. Null-hypothesis probabilities, Cramer's V, and the Contingency Coefficient $C$ are a few of the better known measures of association which may be obtained from the contingency table analysis described below.

A contingency table analysis of association between two variables proceeds by binning the two-dimensional distribution into an $I \times J$ grid [49]. If $N_{i j}$ is the number of points in bin $i, j$, then the row and column totals are

$$
N_{i .}=\sum_{j}^{J} N_{i j}, \quad N_{\cdot j}=\sum_{i}^{I} N_{i j},
$$

and the total number of points in the distribution is

$$
N=\sum_{i, j} N_{i j}=\sum_{i}^{I} N_{i}=\sum_{j}^{J} N_{\cdot j} .
$$

The null hypothesis is the assumption of no association between the two variables. By measuring the fit of this assumption to the data we can learn about the significance of the association. Under the null hypothesis, the fraction of points in bin $i, j$, of column $j$ is independent of $j$. That is, 


$$
\frac{n_{i j}}{N_{\cdot j}}=\frac{N_{i} .}{N}, \quad n_{i j}=\frac{N_{i \cdot} N_{\cdot j}}{N},
$$

where $n_{i j}$ is the number of points in bin $i, j$ predicted by the null hypothesis. The $\chi^{2}$ is

$$
\chi^{2}=\sum_{i, j} \frac{\left(N_{i j}-n_{i j}\right)^{2}}{n_{i j}},
$$

and the number of degrees of freedom are

$$
\nu=I J-I-J+1
$$

where the row and column totals used in the hypothesis are subtracted and the addition of one accounts for the equality of the sum of row and column totals.

Knowledge of $\chi^{2} / \nu$ allows an estimate of the probability for the null hypothesis to be true. A small probability indicates significant association between the data. We calculate the standard null-hypothesis significance parameter

$$
P_{\mathrm{NH}}=\frac{1}{\Gamma(\nu / 2)} \int_{\chi^{2} / 2}^{\infty} e^{-t} t^{\nu / 2-1} d t
$$

where $\Gamma$ is the gamma function.

Cramer's $V$ is a normalized measure of the strength of association independent of the size of the contingency table

$$
V=\left(\frac{\chi^{2}}{N \min (I-1, J-1)}\right)^{1 / 2}
$$

This measure lies in the range

$$
0 \leq V \leq 1
$$

$V=1$ indicates there is a unique square for each $i, j$ holding all points in row $i$ and column j. $V=0$ indicates there is no association.

The Contingency Coefficient $C$ is also a measure of the strength of the correlation. It is defined as

$$
C=\sqrt{\frac{\chi^{2}}{\chi^{2}+N}},
$$

and is dependent on the size of the contingency table. Since all the contingency tables used in this analysis are the same size, this poses no problem. Its range is

$$
0 \leq C<1
$$

with larger values indicating stronger association.

We also consider the significance of association based on the symmetrical uncertainty coefficient $U$ determined via an examination of the entropy of the distribution [49]. In 
essence this measure describes how much entropy is lost in one parameter given knowledge of the other. This measure is bounded by

$$
0 \leq U \leq 1
$$

and large values of $U$ indicate significant loss of entropy implying significant dependency among the parameters.

Once a distribution has been found to be significant, the linear correlation coefficient is calculated

$$
r=\frac{\sum_{i}\left(x_{i}-\bar{x}\right)\left(y_{i}-\bar{y}\right)}{\left[\sum_{i}\left(x_{i}-\bar{x}\right)^{2}\right]^{1 / 2}\left[\sum_{i}\left(y_{i}-\bar{y}\right)^{2}\right]^{1 / 2}} .
$$

The linear correlation coefficient also measures the strength of the association and lies in the range

$$
-1 \leq r \leq 1
$$

When $r=+1$ the points lie on a perfect line with positive slope, while $r=-1$ indicates the points lie on a perfect line with negative slope. $r=0$ indicates there is no correlation. While the linear correlation coefficient is a poor measure of the significance of correlation, it has the advantage of identifying the sign of significant correlations.

Hence the procedure is as follows. The goodness of fit parameter is utilized to identify associations that are significant. This parameter has the advantage of presenting a clean determination of which associations are significant. Cramer's $V$, the contingency coefficient $C$, and the symmetrical uncertainty coefficient are used to determine which QCD parameters play the dominant role in determining hadronic spectral properties. Finally, the proportionality of the association is measured by the linear correlation coefficient. Since the uncertainties of the QCD parameter distributions can affect the strengths of the associations, we utilize 1000 QCD parameter sets in which all QCD parameters have an equal relative uncertainty of $15 \%$. However, the illustrations of this section show the actual correlations for the QCD parameters described in Section [II].

\section{B. $\rho$-meson Associations}

Table III identifies the QCD parameters having significant correlations with the spectral properties of the $\rho$-meson. Here the quark condensate plays a dominant role in determining all the spectral properties. This is simply a reflection of the fact that it appears squared in the last term of (5.1). The correlations for $\kappa_{\rho}$ show a linearized version of essentially the same correlation. The $\alpha_{s}$ correlations are similar to that for $\kappa_{\rho}$ and reflect the fact that the $\alpha_{s}$ corrections to the identity operator are small relative to one. An interesting aspect is the absence of significant gluon condensate correlations for $f_{\rho}^{2}$ and $w_{\rho}$.

The correlations between the $\rho$-meson mass and two of the more interesting QCD parameters estimated in Section III are illustrated in figures 23 and 24. The relatively large uncertainty in the gluon condensate estimate reveals an anti-correlation with the mass. Qualitatively similar plots hold for the residue and continuum threshold. 


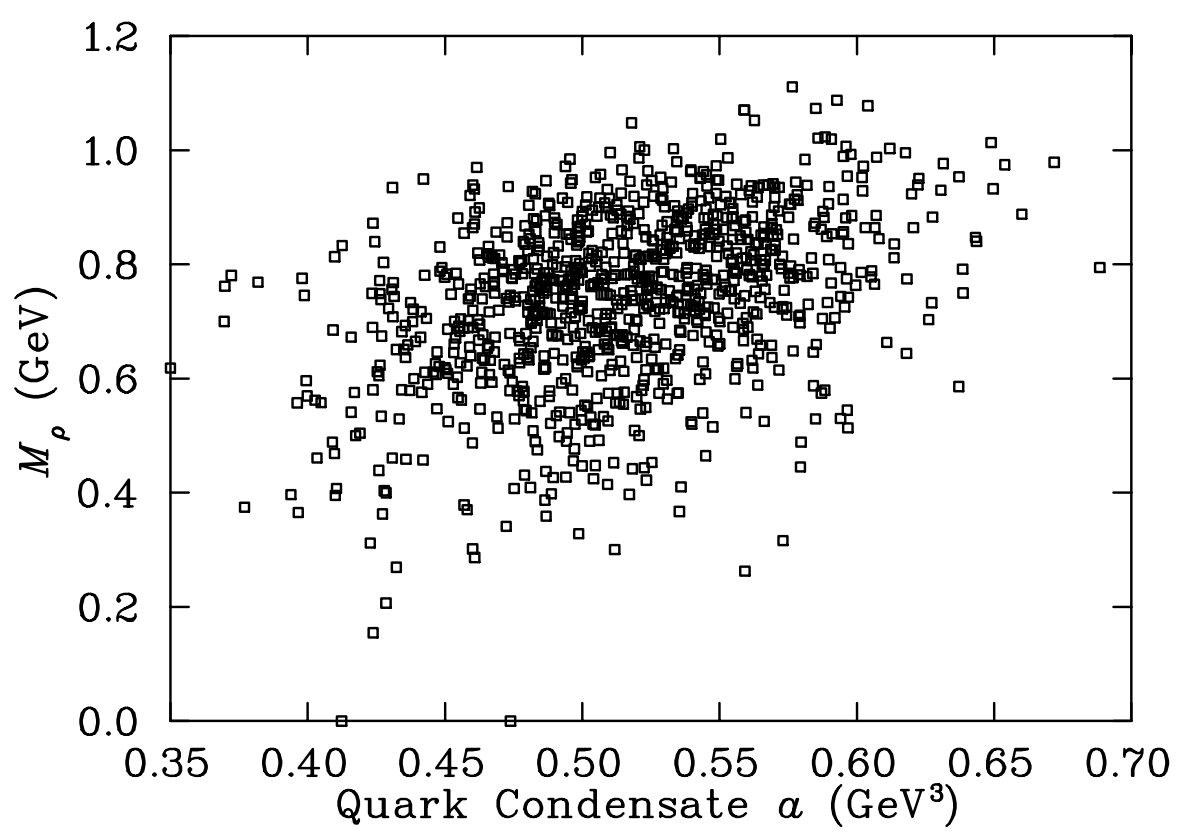

FIG. 23. Scatter plot illustrating the association between the $\rho$-meson mass and the quark condensate $a$ estimated in Section [II].

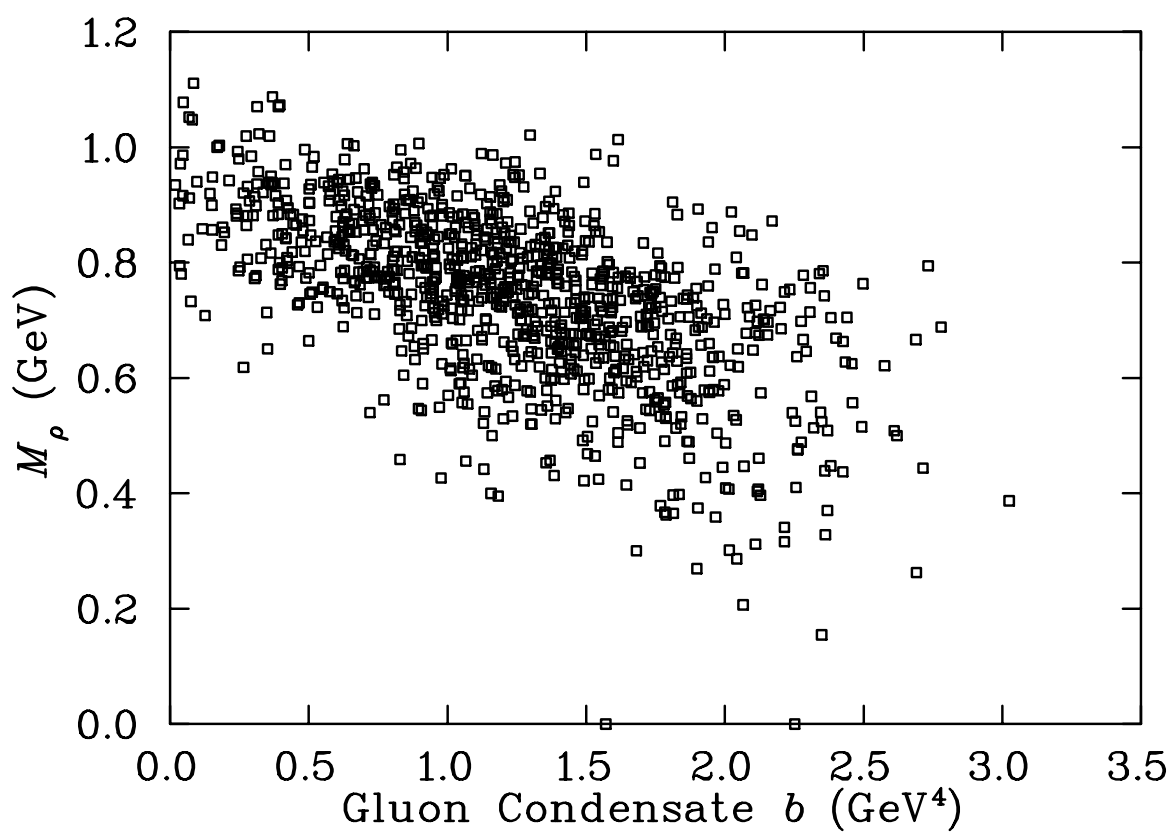

FIG. 24. Scatter plot illustrating the association between the $\rho$-meson mass and the gluon condensate $b$ estimated in Section [II]. 
TABLE III. QCD Parameters having significant roles in determining $\rho$-meson spectral properties. Measures of association include Cramer's $V$, the Contingency Coefficient $C$, the Symmetrical Uncertainty Coefficient $U$, and the linear correlation coefficient $r$.

\begin{tabular}{lccccc}
\hline \hline Spectral Property & Parameter & $V$ & $C$ & $U$ & $r$ \\
\hline$\rho$-meson Mass $M_{\rho}$ & $\langle\bar{q} q\rangle$ & 0.34 & 0.82 & 0.23 & +0.78 \\
& $\left\langle\frac{\alpha_{s}}{\pi} G^{2}\right\rangle$ & 0.20 & 0.63 & 0.07 & -0.27 \\
& $\kappa_{\rho}$ & 0.19 & 0.62 & 0.08 & +0.37 \\
\hline Threshold $w_{\rho}$ of (5.1) & $\alpha_{s} / \pi$ & 0.18 & 0.61 & 0.07 & +0.38 \\
& $\langle\bar{q} q\rangle$ & 0.36 & 0.83 & 0.24 & +0.80 \\
& $\alpha_{s} / \pi$ & 0.21 & 0.66 & 0.09 & +0.42 \\
Pole Residue $f_{\rho}^{2}$ of (5.1) & $\kappa_{\rho}$ & 0.19 & 0.62 & 0.08 & +0.37 \\
& $\langle\bar{q} q\rangle$ & 0.34 & 0.82 & 0.23 & +0.78 \\
& $\alpha_{s} / \pi$ & 0.21 & 0.66 & 0.09 & +0.46 \\
& $\kappa_{\rho}$ & 0.19 & 0.62 & 0.08 & +0.36 \\
\hline \hline
\end{tabular}

\section{Nucleon Associations}

Table IV identifies the QCD parameters having significant correlations with the spectral properties of the nucleon obtained from an analysis of (6.1b) at $\beta=-1.2$. Here the gluon condensate plays the dominant role in determining the mass and continuum threshold. The mixed condensate and finally the quark condensate play lesser roles. The predominant role of the quark condensate is to determine the residue of the nucleon pole. Fortunately, the quark condensate and nucleon mass are at least positively correlated.

However, these correlations are dependent on the choice of interpolating field. Table $\square$ identifies the QCD parameters having significant correlations with the spectral properties of the nucleon obtained from the analysis of (7.6b) and (7.6c) at $\beta=0$. This time the quark condensate plays the dominant role in determining the nucleon mass. This role is also

TABLE IV. QCD Parameters having significantly correlated roles in determining nucleon spectral properties via an analysis of $(6.1 \mathrm{~b})$ at $\beta=-1.2$. Measures of association include Cramer's $V$, the Contingency Coefficient $C$, the Symmetrical Uncertainty Coefficient $U$, and the linear correlation coefficient $r$.

\begin{tabular}{lccccc}
\hline \hline Spectral Property & Parameter & $V$ & $C$ & $U$ & $r$ \\
\hline Nucleon Mass $M_{N}$ & $\left\langle\frac{\alpha_{s}}{\pi} G^{2}\right\rangle$ & 0.30 & 0.77 & 0.20 & +0.76 \\
& $\langle\bar{q} g \sigma \cdot G q\rangle$ & 0.22 & 0.66 & 0.09 & -0.47 \\
& $\langle\bar{q} q\rangle$ & 0.23 & 0.68 & 0.09 & +0.46 \\
\hline Threshold $w_{2}$ of $(\overline{6.1 \mathrm{~b}})$ & $\left\langle\frac{\alpha_{s}}{\pi} G^{2}\right\rangle$ & 0.31 & 0.77 & 0.22 & +0.79 \\
& $\langle\bar{q} g \sigma \cdot G q\rangle$ & 0.22 & 0.64 & 0.09 & -0.44 \\
& $\langle\bar{q} q\rangle$ & 0.22 & 0.65 & 0.09 & +0.43 \\
\hline Pole Residue $\lambda_{\mathcal{O}}^{2}$ of $(\sqrt[6.1 \mathrm{~b}]{ })$ & $\langle\bar{q} q\rangle$ & 0.30 & 0.78 & 0.17 & +0.69 \\
& $\left\langle\frac{\alpha_{s}}{\pi} G^{2}\right\rangle$ & 0.24 & 0.70 & 0.15 & +0.63 \\
& $\langle\bar{q} g \sigma \cdot G q\rangle$ & 0.16 & 0.56 & 0.07 & -0.29 \\
\hline \hline
\end{tabular}


TABLE V. QCD Parameters having significantly correlated roles in determining nucleon spectral properties via a simultaneous analysis of (7.6b) and (7.60) at $\beta=0$. Measures of association include Cramer's $V$, the Contingency Coefficient $C$, the Symmetrical Uncertainty Coefficient $U$, and the linear correlation coefficient $r$.

\begin{tabular}{lccccc}
\hline \hline Spectral Property & Parameter & $V$ & $C$ & $U$ & $r$ \\
\hline Nucleon Mass $M_{N}$ & $\langle\bar{q} q\rangle$ & 0.31 & 0.79 & 0.18 & -0.68 \\
& $\langle\bar{q} g \sigma \cdot G q\rangle$ & 0.26 & 0.73 & 0.17 & +0.67 \\
& $\left\langle\frac{\alpha_{s}}{\pi} G^{2}\right\rangle$ & 0.16 & 0.54 & 0.07 & +0.21 \\
\hline Threshold $w_{3}$ of & $\langle\bar{q} q\rangle$ & 0.32 & 0.80 & 0.17 & -0.66 \\
$(7.6 \mathrm{~b})$ and $(\overline{7.60})$ & $\langle\bar{q} g \sigma \cdot G q\rangle$ & 0.26 & 0.71 & 0.15 & +0.64 \\
& $\left\langle\frac{\alpha_{s}}{\pi} G^{2}\right\rangle$ & 0.16 & 0.55 & 0.08 & +0.52 \\
\hline Pole Residue $\lambda_{\mathcal{O}} \lambda_{3 / 2}$ & $\langle\bar{q} g \sigma \cdot G q\rangle$ & 0.34 & 0.82 & 0.25 & +0.82 \\
of (7.6b) and $(\overline{7.60})$ & $\langle\bar{q} q\rangle$ & 0.17 & 0.56 & 0.08 & -0.37 \\
& $\left\langle\frac{\alpha_{s}}{\pi} G^{2}\right\rangle$ & 0.16 & 0.56 & 0.07 & +0.33 \\
\hline \hline
\end{tabular}

shared with the mixed condensate $\langle\bar{q} g \sigma \cdot G q\rangle$. Perhaps the most interesting result is that the magnitude of the quark condensate is anticorrelated with the nucleon mass, in complete contradiction with the "Ioffe formula". Figures 25, 26 and 27 illustrate the correlations between the nucleon mass and the quark, gluon, and mixed condensates respectively.

What this indicates is that the intimate relationship between the quark condensate and the nucleon mass suggested in the "Ioffe formula" is invalid. Considering the assumptions made in deriving the "Ioffe formula" one might expect the quark condensate value to be tied more tightly to the continuum model than the ground state pole, and this is borne out in Table $\nabla$. There the quark condensate plays the dominant role in determining the continuum model threshold.

Of course, all the condensate values themselves are correlated by the theory of QCD, and this correlation has not been included in this analysis. It may very well be that the nucleon mass is positively correlated with the quark condensate when the higher dimension condensates are also correlated with the quark condensate. The important point to recognize is that the correlation does not come about through some direct connection such as the "Ioffe formula".

Moreover, we are extracting nucleon properties in a Borel regime in which these leading order condensates just happen to be the dominant ones. One could also extract the mass from much deeper in the non-perturbative regime, perhaps by a lattice QCD calculation. There, it is much higher dimension operators which account for the same nucleon mass [3]. Therefore, it is really impossible to identify a particular condensate for giving rise to ground state hadron properties.

The only true statement that may be made about the relationship between condensates and spectral properties is what we have known all along: The condensates reflecting chiral symmetry breaking play a significant role in determining hadron properties. 


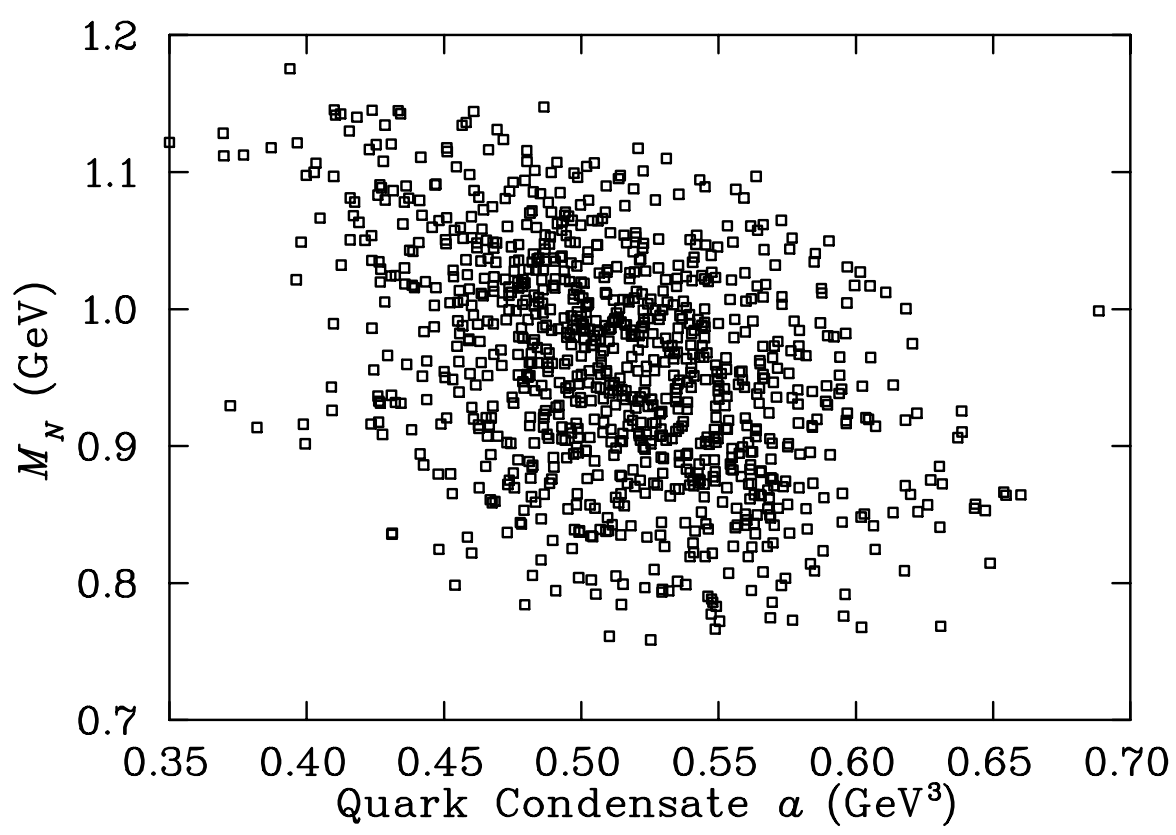

FIG. 25. The association between the nucleon mass and the quark condensate $a$ estimated in Section III. The nucleon mass is extracted from the simultaneous analysis of sum rules (7.6b) and (7.60) at $\beta=0$.

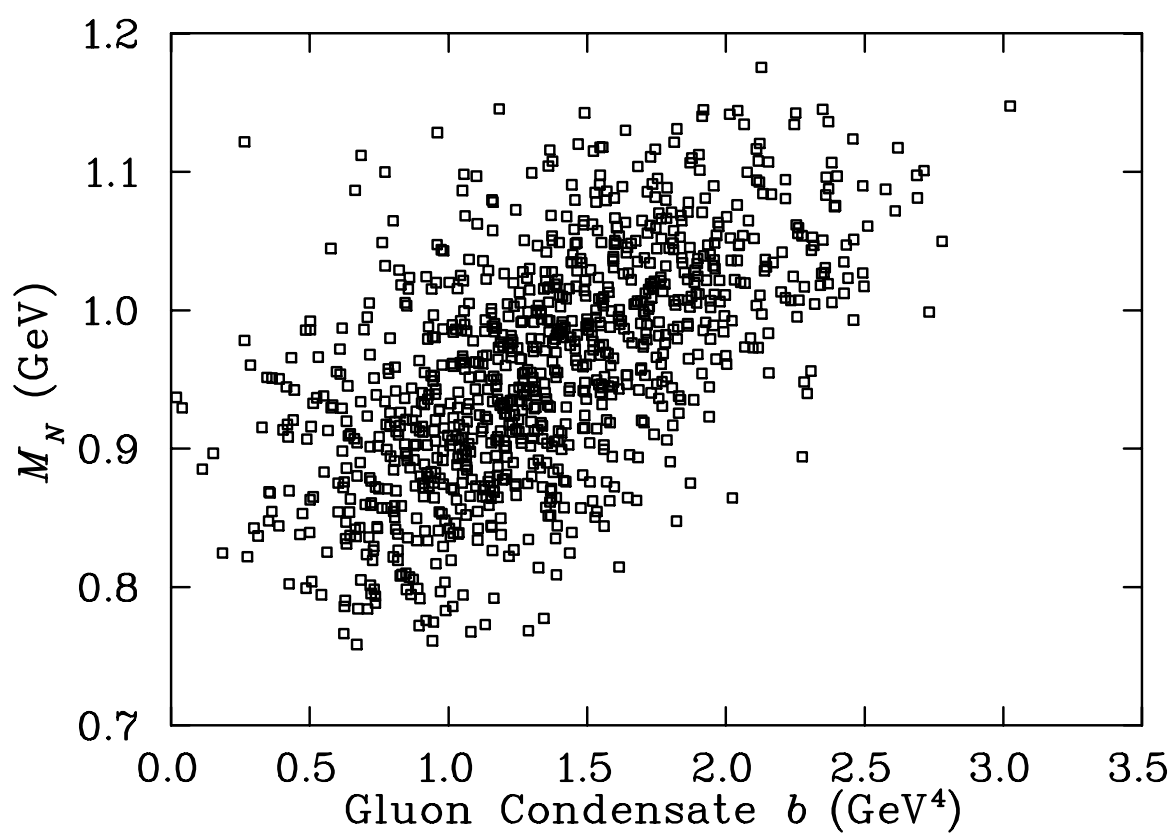

FIG. 26. The association between the nucleon mass and the gluon condensate $b$ estimated in Section III. The nucleon mass is extracted from the simultaneous analysis of sum rules (7.6b) and (7.60) at $\beta=0$. 


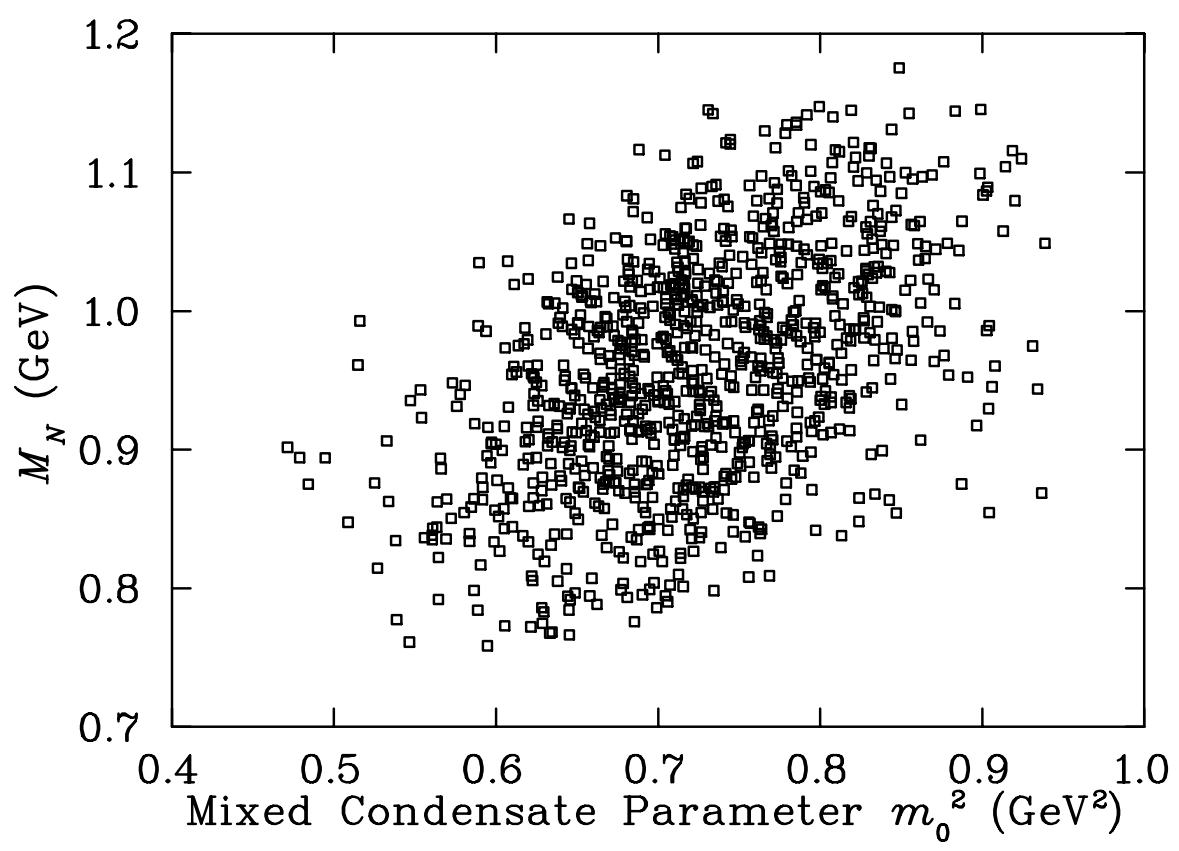

FIG. 27. The association between the nucleon mass and the mixed condensate parameter $m_{0}^{2}$ estimated in Section III. The nucleon mass is extracted from the simultaneous analysis of sum rules $(7.6 \mathrm{~b})$ and $(\overline{7.6 \mathrm{~d})}$ at $\beta=0$.

\section{Phenomenological Correlations}

Correlations among the phenomenological fit parameters themselves are qualitatively different from the correlations with the QCD parameters. Figures 28, 29 and 30 display the close correspondence between all three of the fit parameters, $w_{\rho}, f_{\rho}$, and $M_{\rho}$ obtained from 1000 fits of (5.1). This close association between the $\rho$-meson spectral parameters suggests an underlying constraint on the fit parameters. Indeed, local duality necessarily leads to correlations among the spectral parameters. To examine the correlations of local duality in an analytical but qualitative fashion, consider the finite energy sum rules (FESR) 69 obtained from

$$
\int_{0}^{s_{0}} \phi(s) \rho_{V}(s) d s=\frac{1}{\pi} \int_{0}^{s_{0}} \phi(s) \operatorname{Im} \Pi(s+i \epsilon) d s .
$$

Here, $\rho_{V}(s)$ is given by (2.12b) and $\Pi\left(q^{2}\right)$ is the QCD evaluation of (2.1) at the structure $g_{\mu \nu}-p_{\mu} p_{\nu} / p^{2}$. The weighting function $\phi(s)$ for FESR is taken to be $1, s, s^{2}, \ldots$ These functions weight excited state contributions in favor of ground state contributions and, as such, FESR are inappropriate for a quantitative determination of ground state properties. However, the first moment and, to a lesser extent, the second moment of the FESRs can provide some general insight into the relationships among the spectral parameters. Taking $\phi(s)=1$ and $\phi(s)=s$ yields the following two relationships

$$
\begin{aligned}
8 \pi^{2} f_{\rho}^{2}-s_{0} & =0 \\
8 \pi^{2} f_{\rho}^{2} M_{\rho}^{2}-\frac{s_{0}^{2}}{2} & =8 \pi^{2} m_{q}\langle\bar{q} q\rangle+\frac{\pi^{2}}{3}\left\langle\frac{\alpha_{s}}{\pi} G^{2}\right\rangle \simeq 0 .
\end{aligned}
$$




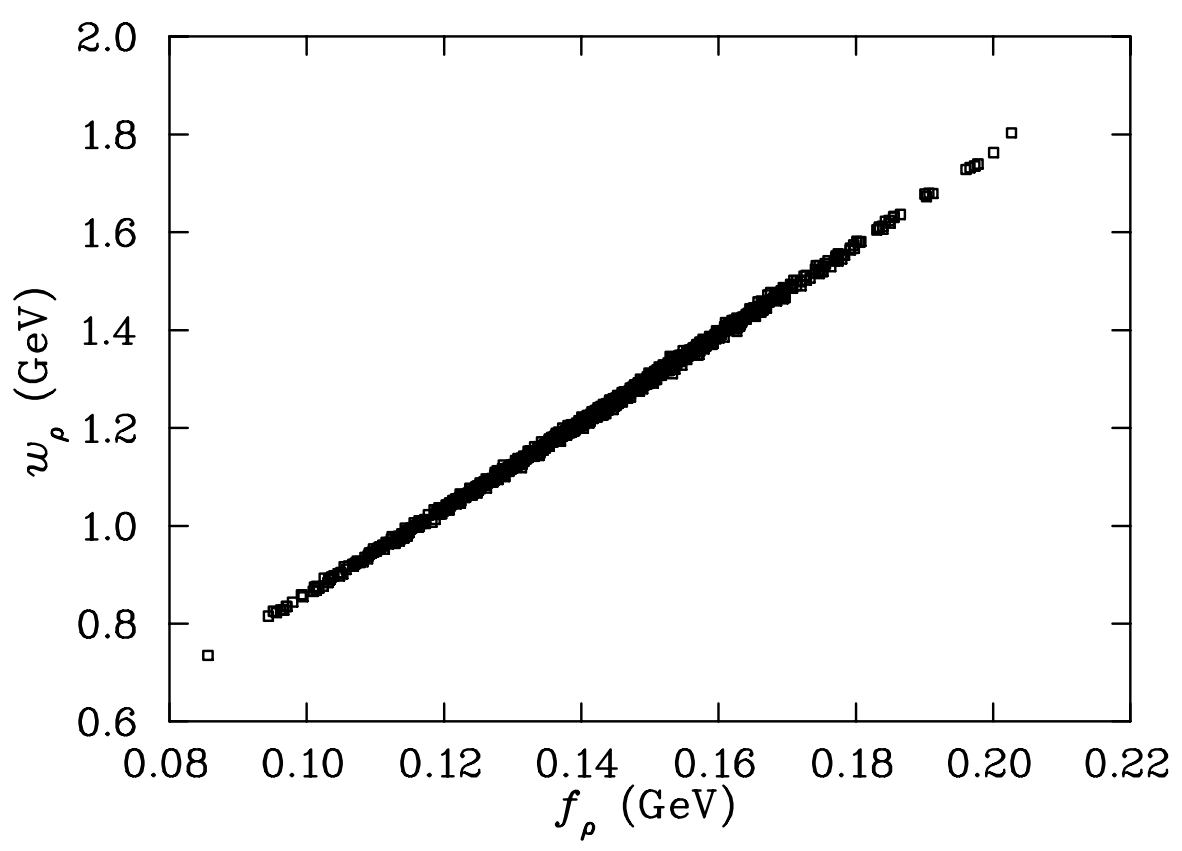

FIG. 28. The association between the $\rho$-meson continuum threshold and the decay constant obtained from 1000 fits of (5.1).

In a qualitative sense, the contributions from the OPE on the right-hand side of $8.15 \mathrm{~b}$ are negligible. In this case (8.15) implies

$$
\begin{aligned}
w_{\rho} & =2 \pi \sqrt{2} f_{\rho}, \\
M_{\rho} & =\frac{1}{\sqrt{2}} w_{\rho}, \\
M_{\rho} & =2 \pi f_{\rho} .
\end{aligned}
$$

These relationships, capturing the features of local duality in a qualitative sense, are independent of the precise values of the QCD parameters. The relationships remain prominent in the more quantitative Borel sum rule analysis as indicated in figures 28, 29 and 30. Of course, the Borel analysis is done independent of these qualitative constraints. The first relation, the most reliable FESR relation, is satisfied remarkably well.

An even more striking correlation is illustrated in figure 31 for the nucleon sum rule of (6.1b) with the optimal $\beta=-1.2$. The association between the continuum threshold and the nucleon mass is nearly one-to-one despite the underlying scatter of QCD parameter values. The explanation for this phenomena is that there is a single term in (6.1b) which is capable of resolving the pole from the continuum. The two leading terms of the OPE are used in deriving the continuum model. Hence if the third term is vanishingly small, the best fit is most certainly pure continuum $w_{2} \rightarrow 0$ as the sum rule is satisfied as a perfect Laplace transform. The few points lying above the curve in figure 31 where the mass equals the threshold are cases where the third term in the sum rule has failed to resolve the pole from the continuum. Figure 32 displays the corresponding association for the mass and the residue of the pole. 


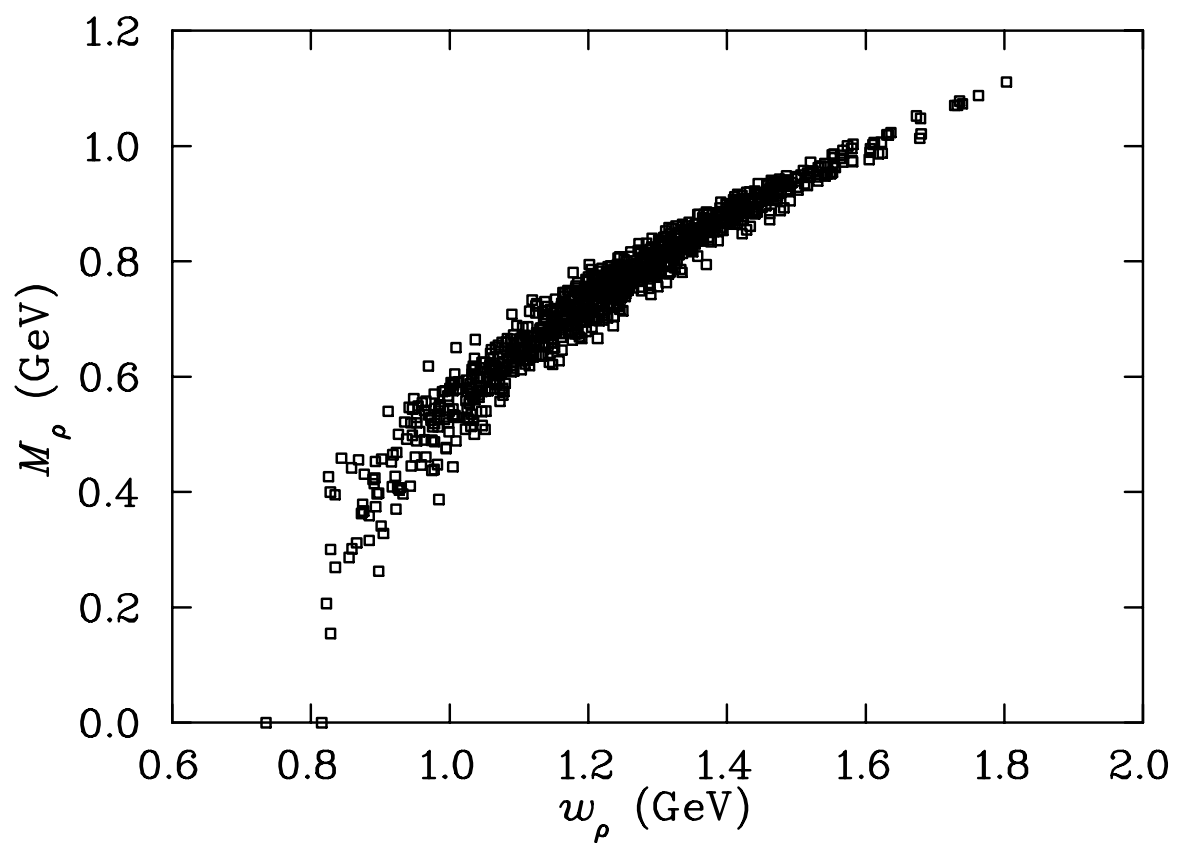

FIG. 29. The association between the $\rho$-meson mass and the continuum threshold obtained from 1000 fits of (5.1).

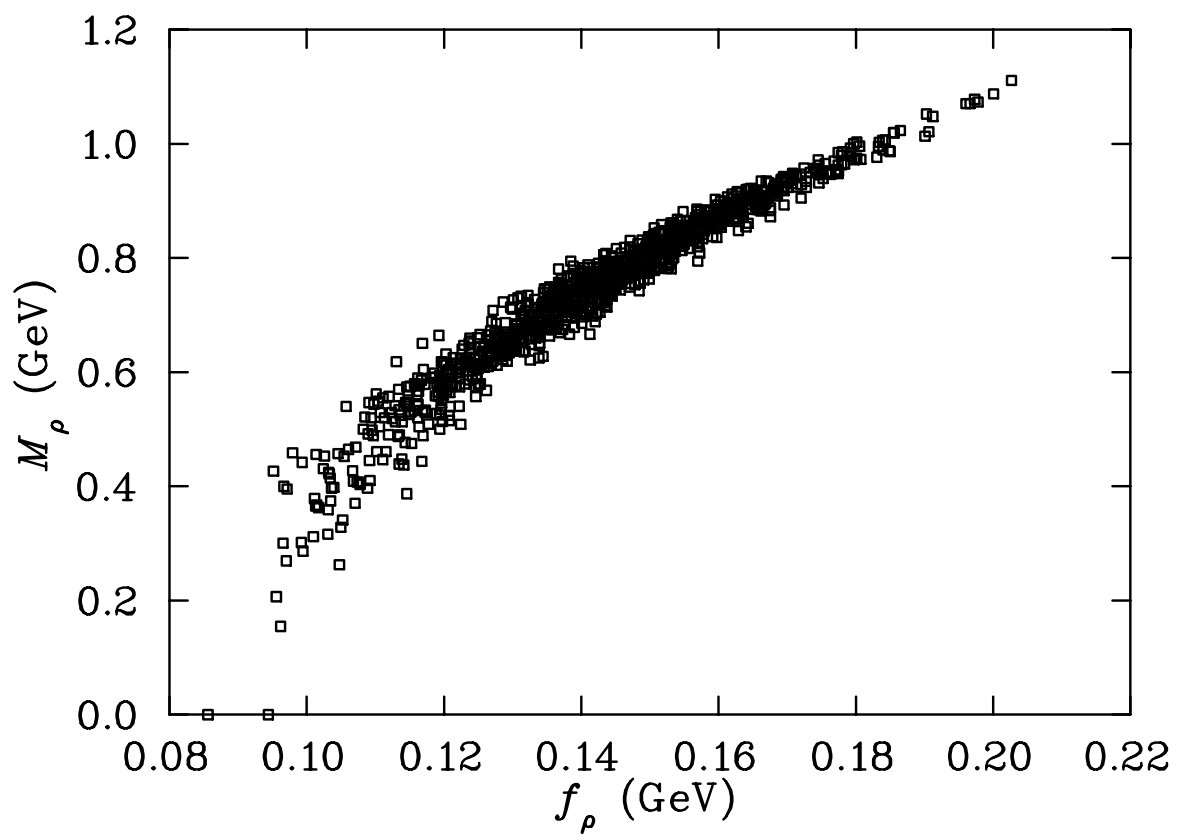

FIG. 30. The association between the $\rho$-meson mass and the decay constant obtained from 1000 fits of (5.1). 


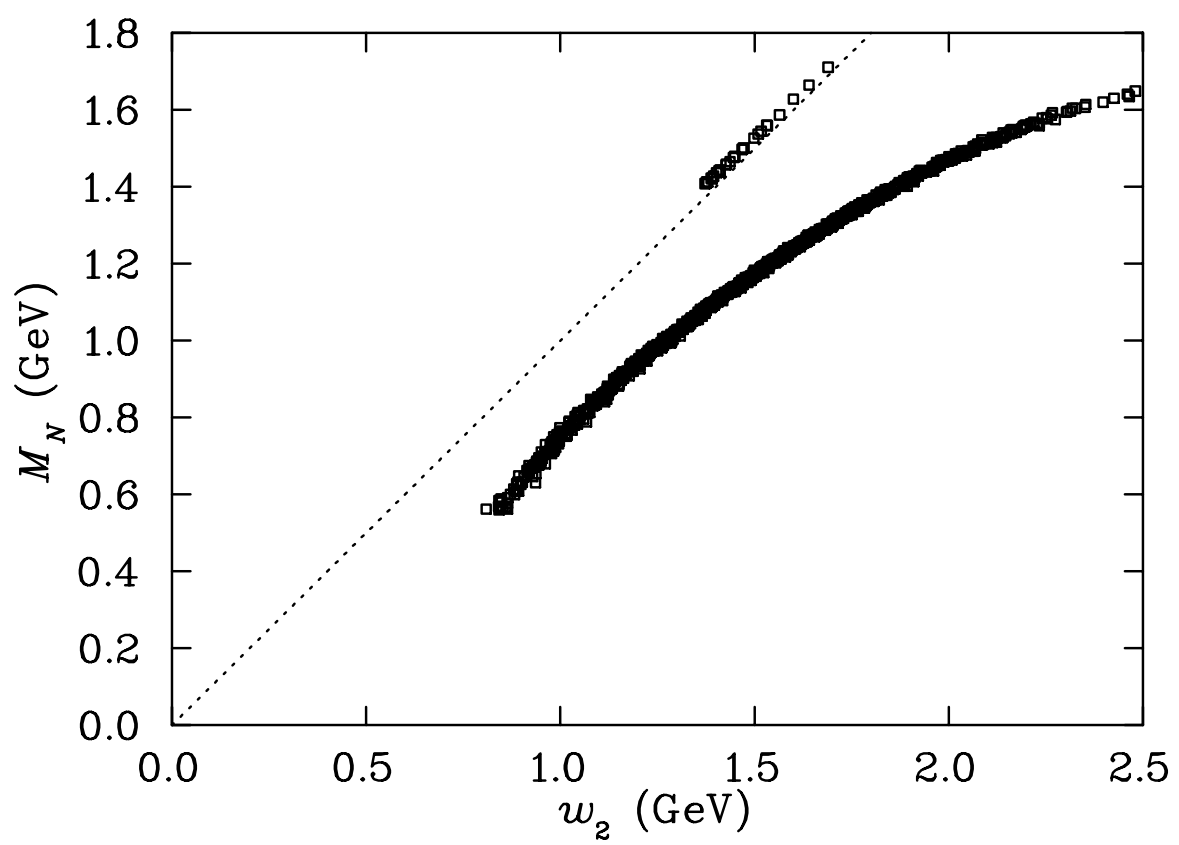

FIG. 31. The association between the nucleon mass and the continuum threshold obtained from 1000 fits of $(6.1 b)$ for the optimal $\beta=-1.2$. The dashed line indicates the equivalence of the mass and threshold.

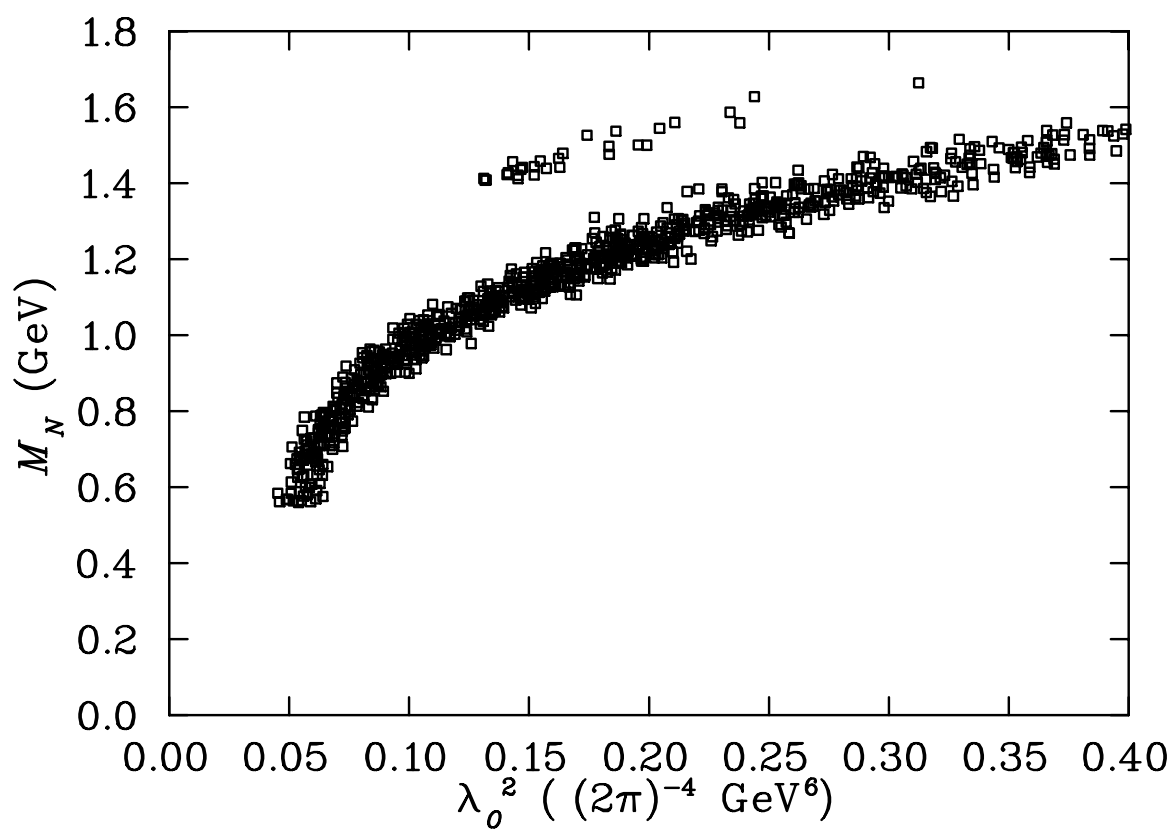

FIG. 32. The association between the nucleon mass and the residue of the pole obtained from 1000 fits of $(6.1 \mathrm{~b})$ for the optimal $\beta=-1.2$. 
In the $\rho$-meson case there are in fact two terms independent of the continuum model in (5.1). Both terms act together to resolve the pole and continuum and hence give rise to some scatter in the mass-threshold association. Scatter in the residue of the pole reflects the fact that all terms affect the strength of the pole.

The strong associations among nucleon spectral parameters reflect the underlying constraints of local duality. Relationships analogous to the FESR for the $\rho$ meson may be derived for the nucleon spectral parameters [69]. However, the larger continuum model contributions to the nucleon correlator make the use of FESR less desirable. The approximate nature of the continuum model may be too crude for a weighting function that fails to suppress excited state strength in the spectral density. The resultant FESR relations are relatively crude and do not capture the essence of local duality as well as in the $\rho$-meson case.

A very different picture develops for the correlations among the nucleon fit parameters obtained from the analysis of (6.1b) for the unfavorable value of $\beta=+0.8$. Figure 33 displays the correlation between the mass and the continuum threshold, $w_{2}$. The corresponding correlation between the mass and the residue is illustrated in figure 34 .

The nature of the three regimes discussed earlier in section $\mathrm{VIF}$ is resolved here. The near-pure continuum fits are revealed in the lower-left corner of the mass-threshold scatter plot of figure 33. The collision of the continuum threshold and the nucleon mass gives rise to the intermediate regime where the gap between ground and excited states has not been resolved. The smaller residue compensating for strength from the continuum model in these fits is illustrated in figure 34. The third pole-plus-continuum regime displays the same near one-to-one correspondence between the mass and the threshold as seen in figure 31 for the optimal $\beta=-1.2$.

The correlations among the spectral parameters are interesting because they reveal the manner in which the sum rules work. Correlations among the fit parameters from the simultaneous analysis of $(7.6 \mathrm{~b})$ and $(7.6 \mathrm{c})$ at $\beta=0$ are illustrated in figures 35 and 36. These distributions are similar to those for the celebrated $\rho$-meson sum rule, and reflect the presence of two terms separating pole from continuum.

\section{ON THE NECESSITY OF DIRECT INSTANTONS}

Arguments favoring the need for direct instanton contributions to the QCD side of QCDSRs [70,60] are based on the assumption that the average instanton size [71], $\rho_{c}=0.33 \mathrm{fm}$ $\ll 1 / \mu=0.39 \mathrm{fm}$, the separation scale of the OPE. Even though $\rho_{c} \sim 1 / \mu$, proponents of direct instanton contributions still argue that the effects of instantons are not sufficiently accounted for in QCD condensates alone.

The analysis of direct instantons in nucleon sum rules are based on many additional assumptions. Here we focus on the most recent investigation of Ref. [60]. There, $\alpha_{s}$ corrections to the identity operator are neglected. As discussed in Section V1 these leading order corrections are $50 \%$ and suggest that $\alpha_{s}$ corrections are completely out of control. Moreover the possibility of a significant dimension-two power correction arising from a summation of the perturbative series, uncertainties surrounding the factorization of the four-quark oper-

ator, coupled with an absence of a valid Borel regime for the sum rule of (6.1a) make any 


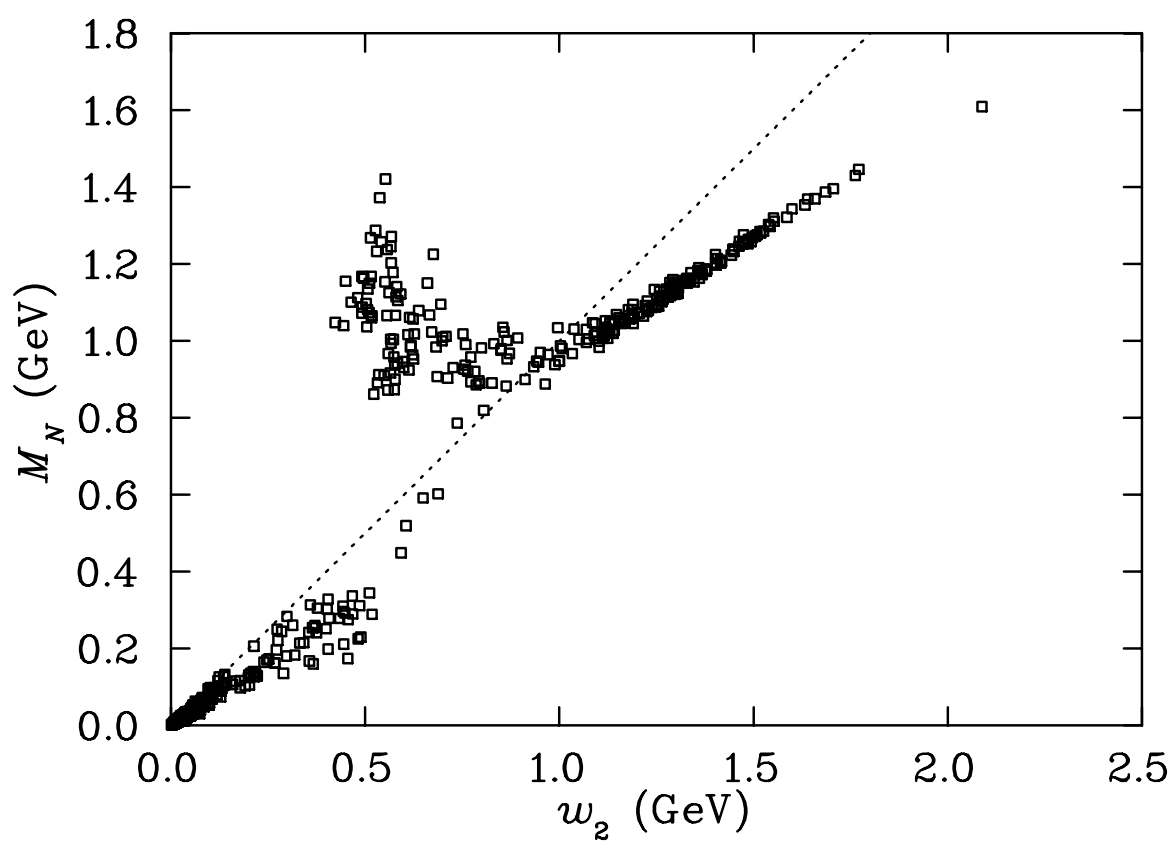

FIG. 33. The correlation between the nucleon mass and the continuum threshold, $w_{2}$, obtained from the analysis of (6.1b) for the unfavorable value of $\beta=+0.8$. The dashed line indicates the equivalence of the mass and threshold.

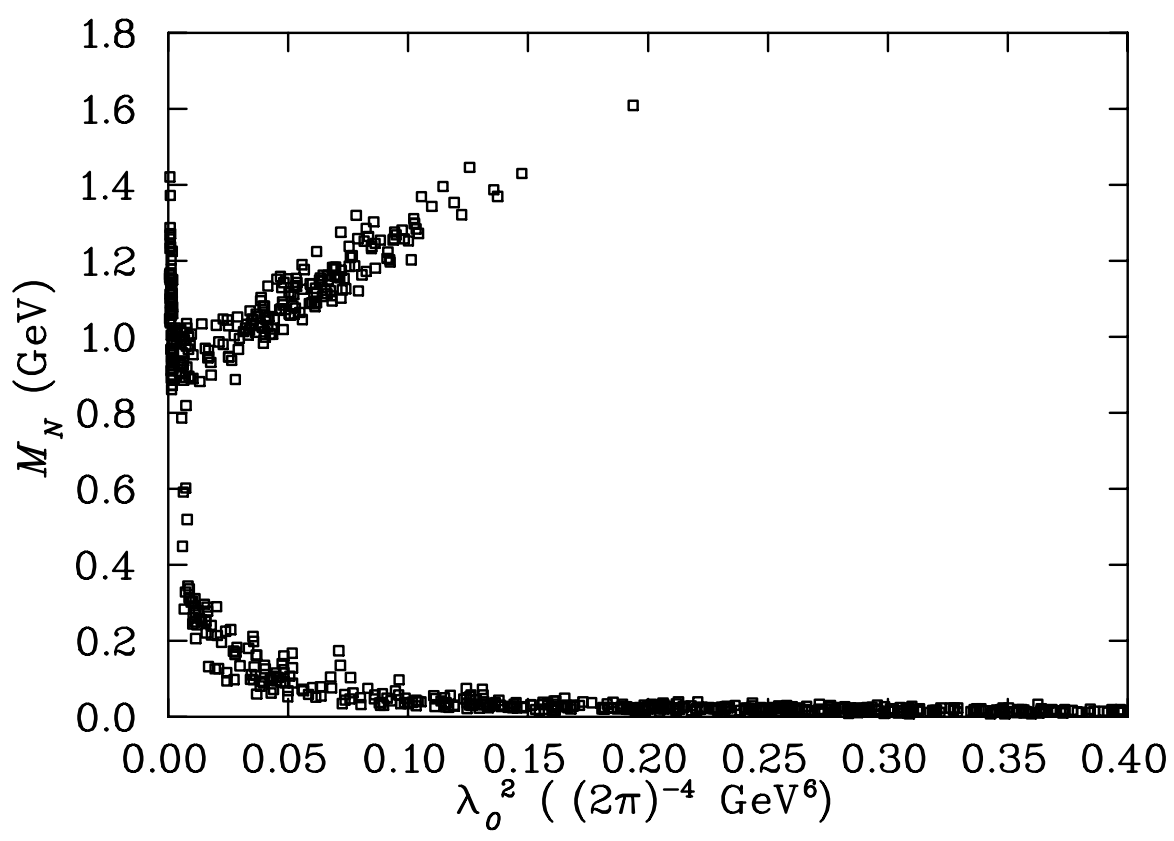

FIG. 34. The correlation between the nucleon mass and the residue of the pole, $\lambda_{\mathcal{O}}$, obtained from the analysis of (6.1b) for the unfavorable value of $\beta=+0.8$. 


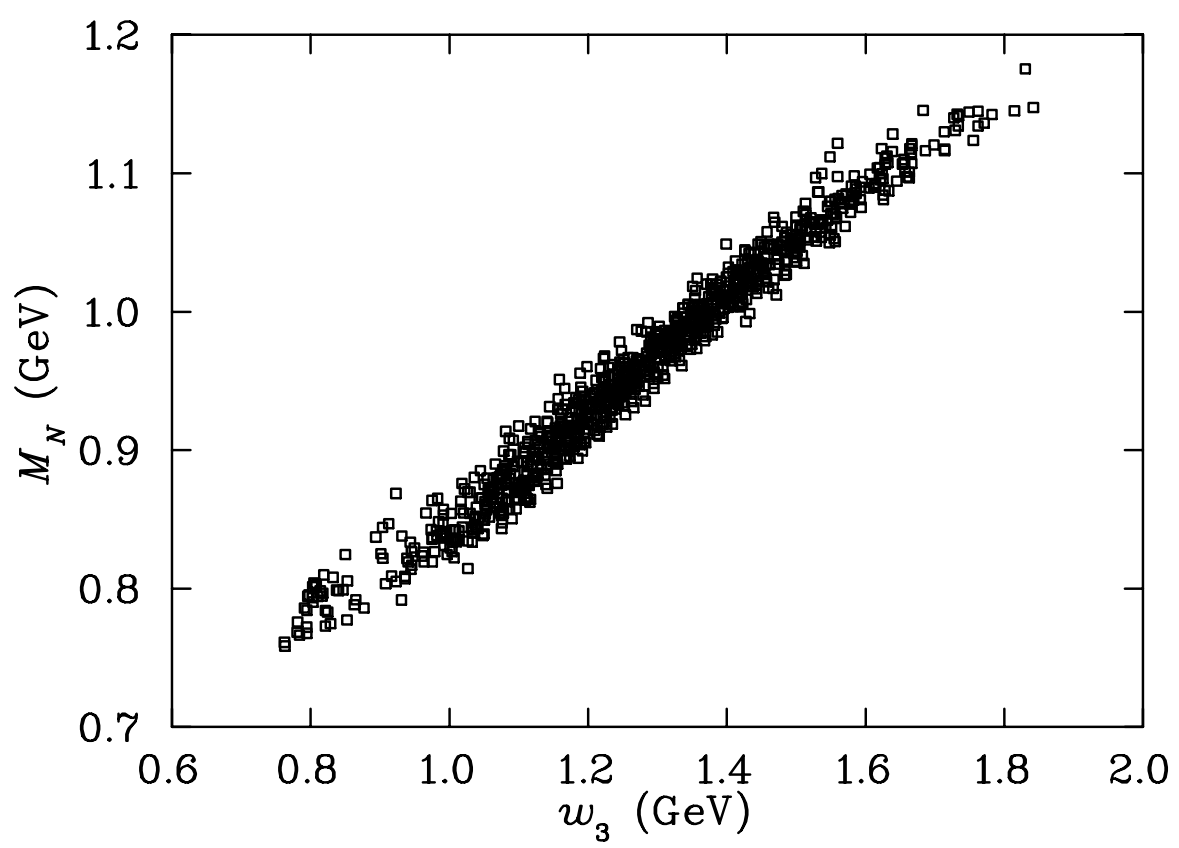

FIG. 35. The correlation between the nucleon mass and the continuum threshold, $w_{3}$, obtained from the simultaneous analysis of sum rules (7.6b) and (7.6c) at $\beta=0$.

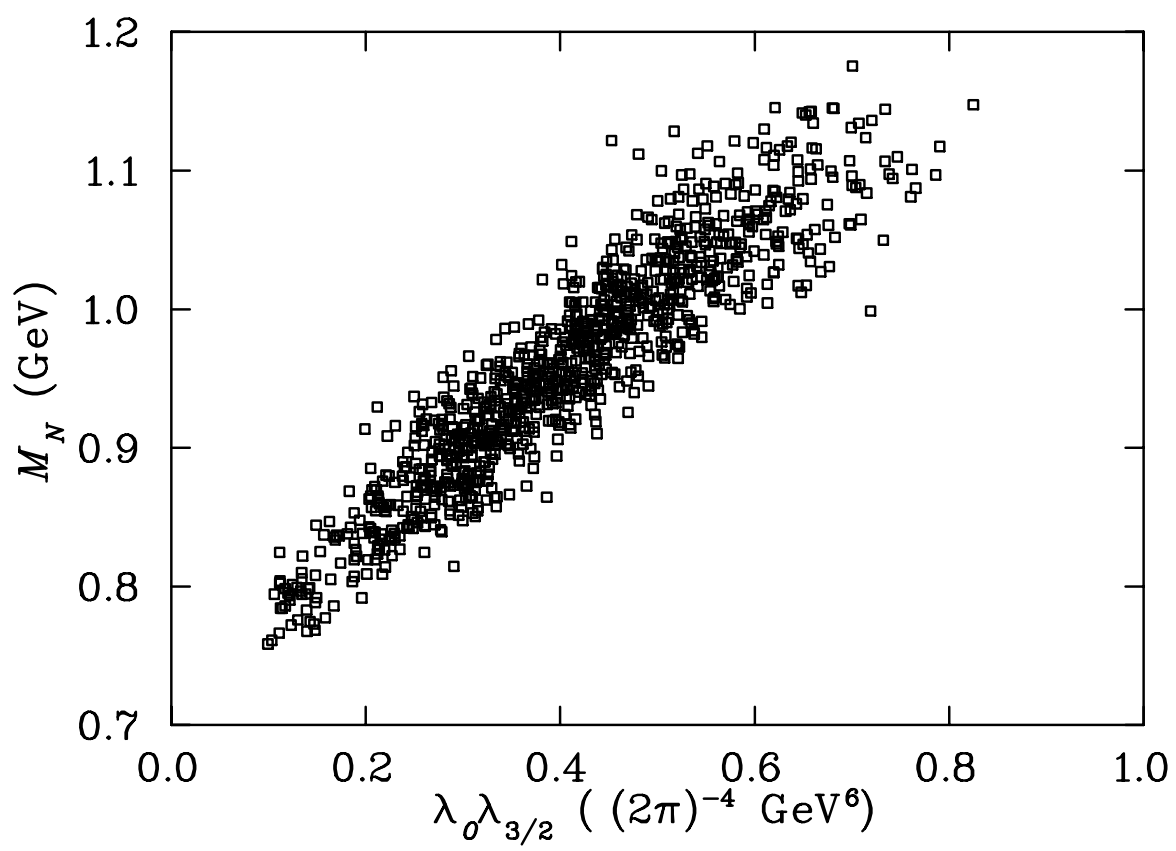

FIG. 36. The correlation between the nucleon mass and the residue of the pole, $\lambda_{\mathcal{O}} \lambda_{3 / 2}$, obtained from the simultaneous analysis of sum rules (7.6b) and $(7.6 \mathrm{~d})$ at $\beta=0$. 
conclusions dubious.

In addition, the OPE is truncated at dimension six. As we saw in Section VI the dimension six, seven, and eight operators are crucial to separating the ground state pole from the continuum. Without the dimension seven term, the best fit of (6.1b) is pure continuum. The fit is perfect when $w_{2} \rightarrow 0$ and $\lambda \rightarrow 0$. Hence, the nucleon sum rule at the structure 1 in Ref. [60 (equation 12) is unstable by design. Any further discussion of stabilizing the sum rule by adding instanton contributions is uninteresting. Finally, issues surrounding the problems of double counting were handled crudely by simply eliminating OPE contributions beyond dimension five. Hence one should not be prejudiced by existing analyses suggesting nucleon sum rules require direct instanton contributions. The issue is really not settled.

For the spin-1/2 interpolator of (2.4a), direct instanton contributions to the sum rule of (6.1b) are governed by the factor [60]

$$
c_{2}=\left(13 \beta^{2}+10 \beta+13\right) / 8 \text {. }
$$

The direct instanton contributions were argued to be of vital importance in Ref. [60] in the vicinity of $\beta \sim-1$.

In the lattice QCD investigation of [13] it was discovered that the overlap of the interpolator $\chi_{2}$ with the nucleon ground state is one hundred times smaller than that for $\chi_{1}$. While the nucleon mass is certainly independent of the interpolating field mixing parameter $\beta$, one also has the residue of the pole, $\lambda_{\mathcal{O}}$, independent of $\beta$ at the $1 \%$ level. This anticipated independence of both ground state properties provides new opportunities for evaluating the necessity of direct instanton effects.

As a first test of sum rule consistency, we utilize the sum rule of (6.1b) to extract the mass as a function of the interpolating field mixing parameter $\beta$. As $\beta \rightarrow-1.5$ the terms contributing to the continuum model become very small and additional poles must be added to the phenomenological side of the sum rule to account for strength in the correlator lying above the ground state mass. As a result we restrict $\beta \geq-1.3$. Valid Borel regimes were found over the range $-1.3 \leq \beta \leq-0.9$. The coefficient $c_{2}$ changes by $50 \%$ over this range. If direct instanton contributions are indeed important at $\beta \sim-1$, the absence of direct instanton contributions in this investigation might be revealed by a significant $\beta$ dependence in the nucleon mass.

Figure 37 displays the ratio of the nucleon mass extracted at $\beta$ to the value obtained at the optimal $\beta=-1.2$. Uncertainties are obtained from two uncorrelated samples of 200 QCD parameter sets. As such the central value at $\beta=-1.2$ need not be 1 . The large uncertainties associated with this sum rule prevent a sensitive test of the necessity of direct instantons. It is clear that all ratios accommodate the ratio of 1 .

A more demanding test may be performed using the sum rules arising from the consideration of the overlap of spin-1/2 and spin-3/2 interpolators. The smaller uncertainties allow a more interesting examination of these issues. In addition, the continuum model terms are independent of $\beta$ and provide a wider range of $\beta$ in which a valid Borel regime exists.

Figure 38 addresses the dependence of the nucleon mass obtained from the sum rules of (7.6b) and (7.6c) on the interpolating field mixing parameter $\beta$. Here, ratios of the mass obtained at finite $\beta$ to $\beta=0$ are displayed. Sum rule consistency demands that all ratios 


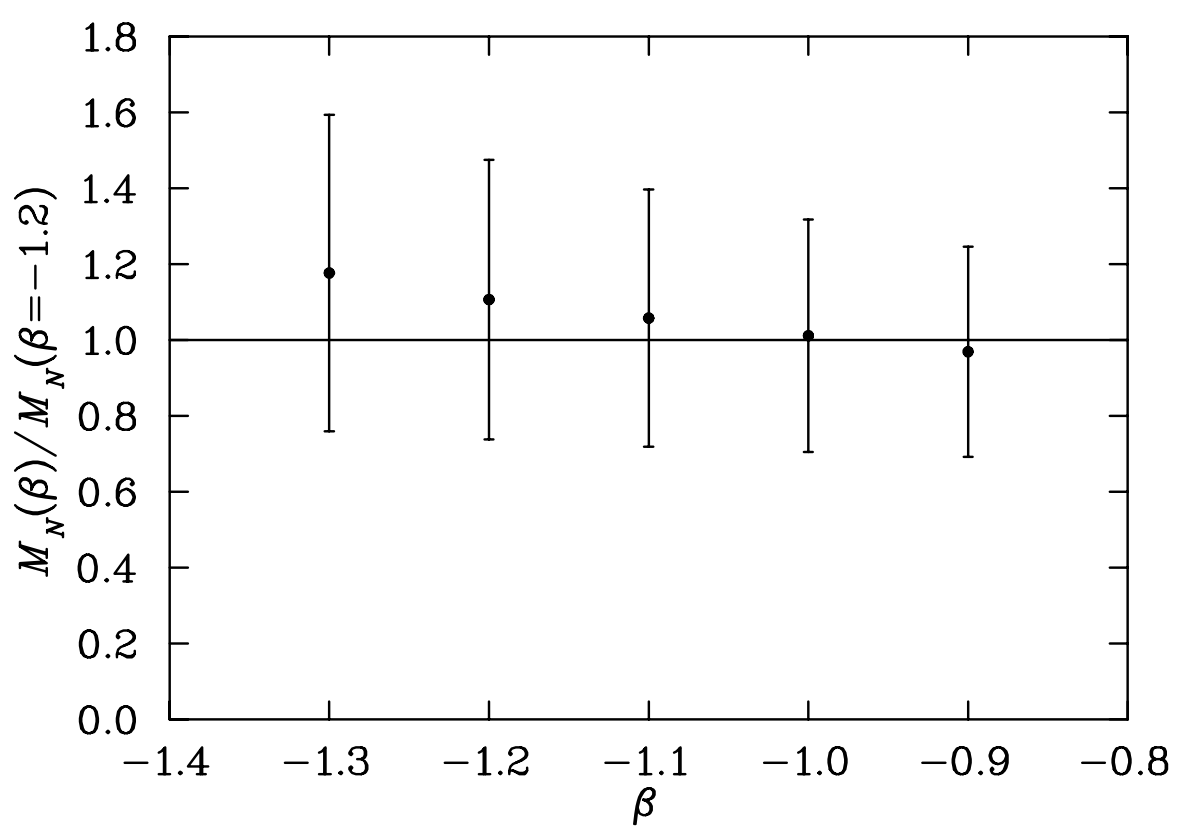

FIG. 37. The dependence of the nucleon mass obtained from the sum rule of $(6.1 \mathrm{~b})$ on the interpolating field mixing parameter $\beta$. Uncorrelated uncertainty estimates are obtained from two samples of 200 QCD parameter sets.

agree with one, and this is satisfied in figure 38 without direct instanton contributions. The uncertainty estimates are obtained from two uncorrelated samples of 200 QCD parameter sets. We note that a valid regime does not exist for $\beta \leq-0.8$. Hence the use of the Ioffe interpolator $(\beta=-1)$ is a particularly unfavorable choice for these sum rules. The large error bar at $\beta=-0.6$ reflects the small valid Borel regime used in the fit.

The corresponding dependence of $\lambda_{\mathcal{O}}$ on $\beta$ is plagued by huge uncertainties. Small changes in the nucleon mass which appears squared in the exponential of the phenomenological side of the sum rules leads to large fluctuations in $\lambda_{\mathcal{O}}$. Hence it is difficult to gain any information on the possible size of direct instanton contributions. However, they are certainly unnecessary as all ratios encompass 1.

In order to proceed, we consider fixing the nucleon mass to the value determined at $\beta=0$ in an attempt to reduce the uncertainty in $\lambda_{\mathcal{O}}$. Figure 39 displays these ratios. The corresponding plot for the continuum threshold is given in figure 40 .

For $\beta<0$ the continuum threshold tends to rise and the distribution of the residue remains largely independent of $\beta$ without direct instanton contributions. For $\beta>0$ the continuum threshold drops to account for strength that would otherwise be absorbed by the rising nucleon mass as illustrated in figure 38. With the threshold near the pole position, strength which might otherwise rest in the pole has been suppressed as discussed in section VIIID. Hence the drop in the residue is an artifact of our fixing the nucleon mass in an attempt to reduce the error bar.

In summary, the independence of the mass and residue from $|\beta| \lesssim 1$ is satisfied within uncertainties in the absence of direct instanton contributions. As the sum rules are improved in the future and QCD input parameters are better determined, it will be important to 


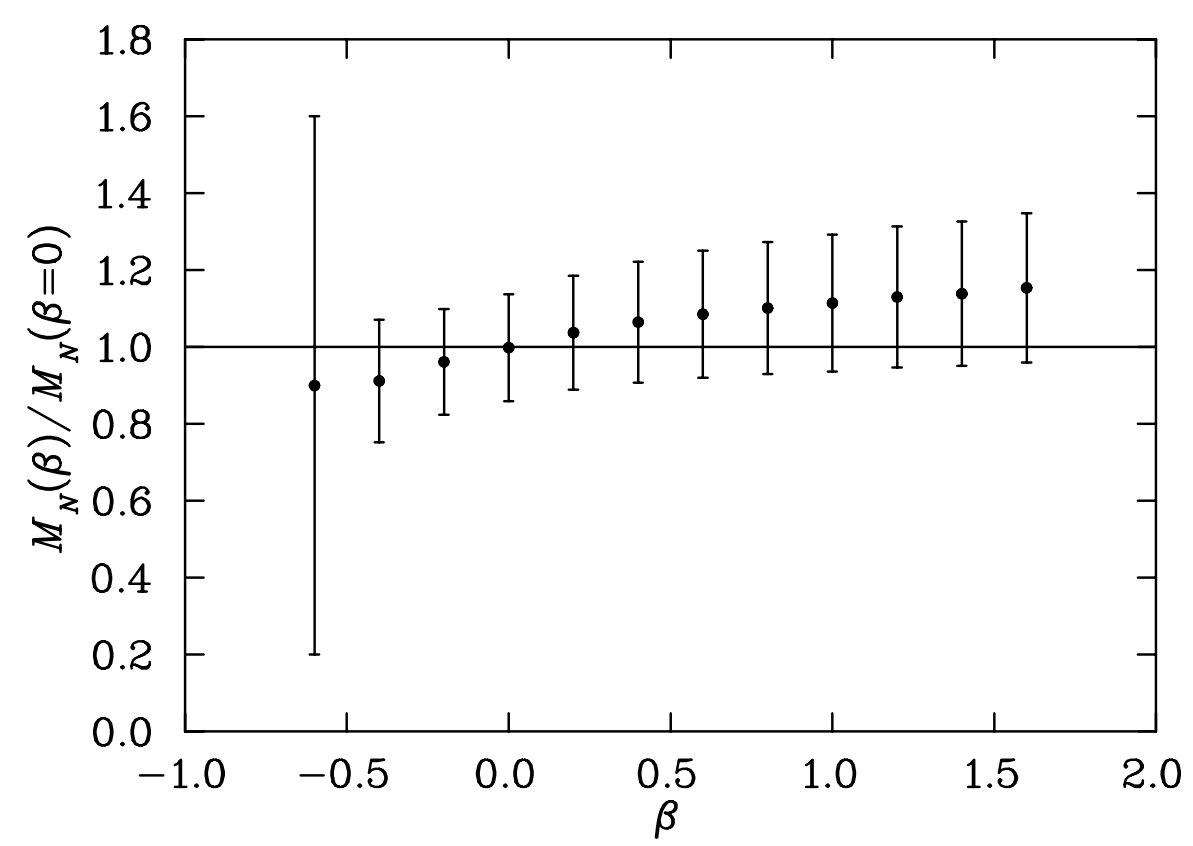

FIG. 38. The dependence of the nucleon mass obtained from the sum rules of (7.6b) and (7.60) on the interpolating field mixing parameter $\beta$. Uncorrelated uncertainty estimates are obtained from two samples of 200 QCD parameter sets.

repeat this analysis to again evaluate the necessity of direct instanton contributions or other refinements of the QCD-SR approach.

At present, direct instanton contributions are not required. This is good news indeed as the amount of modeling required for instanton estimates is relatively unpalatable when compared to that for the standard QCD-SRs themselves. In fact a preferable approach would be to simply raise the OPE separation scale further into the perturbative regime to ensure that direct instanton effects are more fully accounted. However, this does not yet appear to be necessary.

\section{COORDINATE SPACE CORRELATOR IMPLICATIONS}

Ground state properties have been found to be independent of the interpolating field mixing parameter $\beta$. On the other hand, the leading terms of the OPE for (6.1) are very dependent on $\beta$. This situation has interesting ramifications for previous analyses of coordinate space point-to-point correlation functions 62,63,72] where there has been some confusion surrounding the physics represented in point-to-point correlation functions.

In Ref. [63] some conclusions are drawn from an incorrect interpretation of the physics represented in two-point correlators. These authors suggest that the behavior of a ratio of the interacting to free correlator can reveal information on clustering in the scalar diquark channel.

It should be emphasized that the phenomenological description of the two-point function involves the baryon mass, the continuum threshold describing the effective onset of excited 


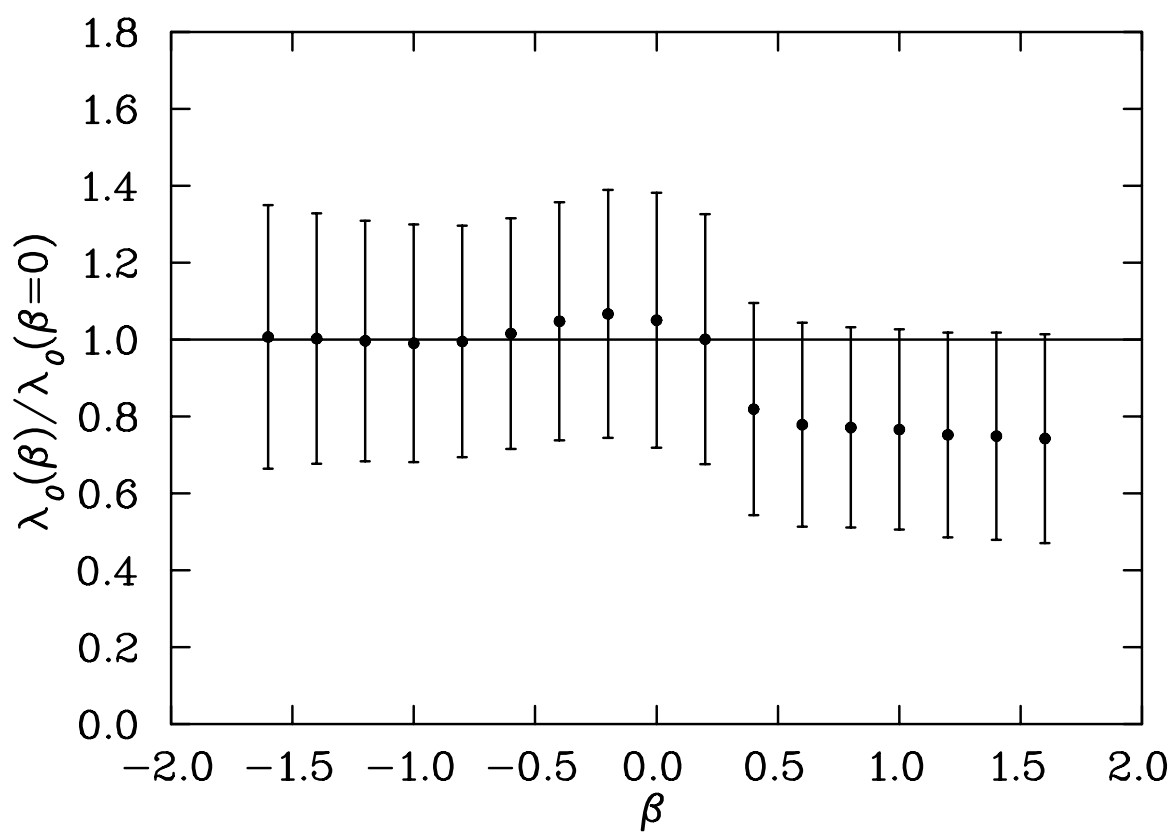

FIG. 39. The dependence of $\lambda_{\mathcal{O}}$ determined by the sum rules of $(7.6 \mathrm{~b})$ and $(7.6 \mathrm{~d})$ on the interpolating field mixing parameter $\beta$. Here the nucleon mass has been fixed to the optimal value obtained at $\beta=0$. Uncorrelated uncertainty estimates are obtained from two samples of $200 \mathrm{QCD}$ parameter sets.

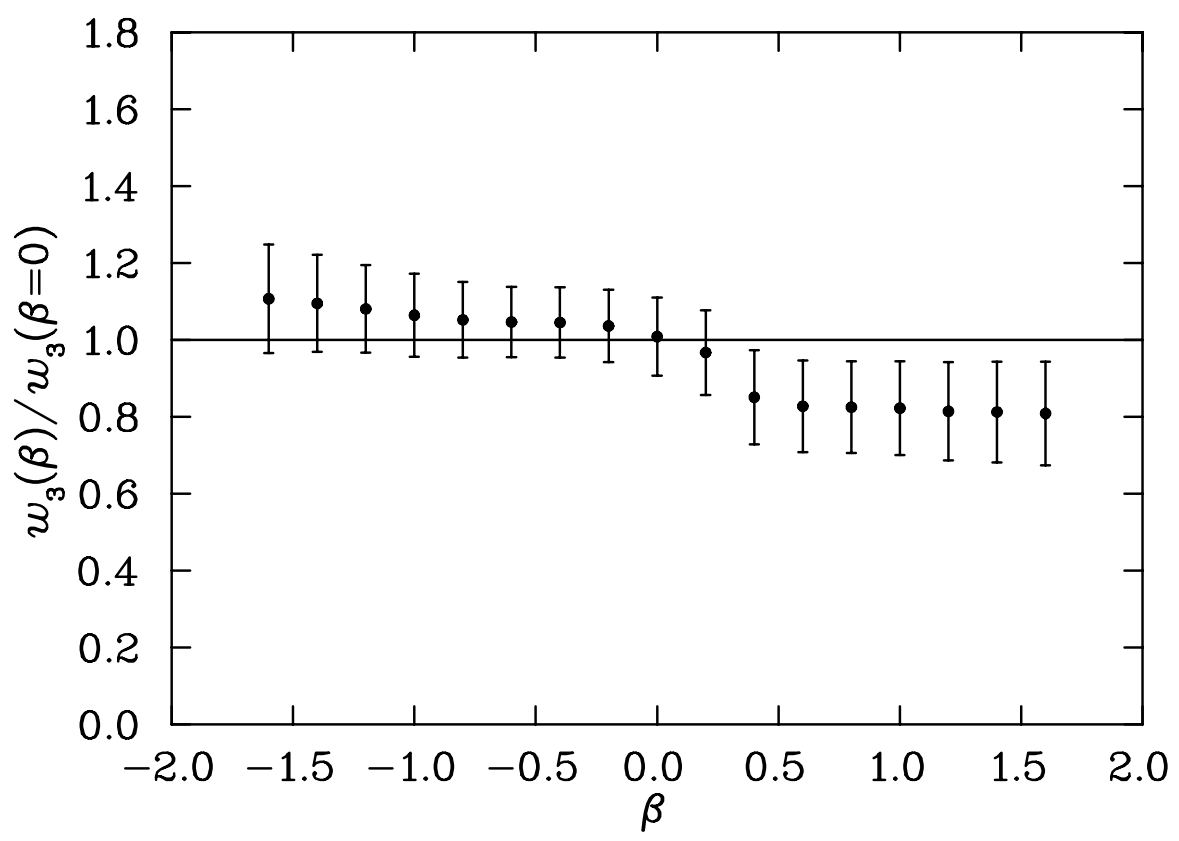

FIG. 40. The dependence of the continuum threshold obtained from the sum rules of (7.6b) and $(7.6 \mathrm{c})$ on the interpolating field mixing parameter $\beta$. Here the nucleon mass has been fixed to the value obtained at $\beta=0$. Uncorrelated uncertainty estimates are obtained from two samples of 200 QCD parameter sets. 
states and the residue of the pole which indicates the ability of the particular interpolator to excite the baryon from the QCD vacuum. Information on quark configurations in the nucleon wave function is absent in point-to-point correlation functions.

The coordinate-space correlator may be obtained from (2.10b) or (2.11b) via

$$
\Pi(x)=\frac{1}{(2 \pi)^{4}} \int d^{4} p e^{-i p \cdot x} \int_{0}^{\infty} \frac{\rho(s)}{s-p^{2}} d s .
$$

The important point here is that for large $x, \Pi(x)$ is dominated by the contribution from the ground state pole. As such $\Pi(x)$ is independent of $|\beta| \lesssim 1$ at the $1 \%$ level, or alternatively $\Pi(x)$ is independent of $|\beta| \lesssim 10$ at the $10 \%$ level $\llbracket 13$.

The coordinate space analyses focused on a ratio of $\Pi(x) / \Pi^{0}(x)$ where $\Pi^{0}(x)$ is the free coordinate-space correlator. For the correlator at the structure $\gamma \cdot p$ studied in 62, 63, 62, at $\beta=-1$, the generalized free correlator is given by

$$
\Pi^{0}(x)=\frac{-3 i}{4 x^{10} \pi^{6}}\left(5+2 \beta+5 \beta^{2}\right) .
$$

Since the denominator is $\beta$ dependent and the numerator is independent of $\beta$ for large $x$, the ratio is dependent on $\beta$. The rate at which the curve turns up as the ground state dominates and the free correlator continues to drop off for increasing $x$ is dependent on the interpolator mixing parameter $\beta$. The rate at which the ratio increases above 1 describes the relative weightings of ground versus excited states generated by the interpolating field. It has nothing to do with the possibility of scalar diquark degrees of freedom being predominant in the nucleon wave function. Moreover, a comparison with a $\Delta^{+}$correlation function for example has no meaning.

\section{CONCLUSIONS}

A rigorous procedure for extracting quantities of phenomenological interest from QCD Sum Rules has been established. We have performed a comprehensive analysis of ground state $\rho$-meson and nucleon QCD sum rules. The inclusion of uncertainty estimates for the phenomenological parameters has allowed us to evaluate the predictive ability and self consistency of the QCD-SRs. When the analysis is done rigorously, QCD Sum Rules are predictive.

Some have argued that fitting sum rules appears to be more of an art than a science. Indeed, many of the findings here contradict the conventional wisdom of both practitioners and skeptics alike [73] and suggests that past arguments have been based more on rhetoric than sound scientific arguments.

The analysis of thousands of QCD parameter sets reveals the true predictions of the sum rules. For the nucleon the results may be summarized as follows.

- The nucleon sum rule of (6.1a), traditionally taken to be the most reliable sum rule, fails to have a Borel regime where both the truncated OPE is reasonably convergent and the ground state dominates the phenomenology. 
- The nucleon sum rule of (6.1b), also obtained from spin-1/2 interpolating fields, resolves the nucleon mass to $\pm 260 \mathrm{MeV}$. This uncertainty is more than double the value commonly assumed by most practitioners.

- The nucleon sum rules of $(7.6 \mathrm{~b})$ and $(\overline{7.6 \mathrm{~g}})$ obtained from the consideration of a spin$3 / 2$ isospin- $1 / 2$ interpolator projected by the generalized spin- $1 / 2$ isospin- $1 / 2$ interpolator are much more reliable than the traditional sum rules. They have greater overlap with the ground state pole relative to the continuum model, have broader valid Borel regimes and provide phenomenological estimates of nucleon spectral properties which are more stable than those obtained from the conventional sum rules.

- The nucleon mass obtained from a simultaneous analysis of (7.6b) and (7.6d) at the optimal $\beta=0$ is resolved to $\pm 80 \mathrm{MeV}$ using $\mathrm{QCD}$ parameter estimates from the literature.

- The dependence of the spectral parameters on the interpolating field mixing parameter is in accord with expectations without resort to direct instanton contributions. This is compelling evidence that instanton physics is adequately accounted for in the nonperturbative vacuum expectation values.

The latter point is truly exciting for the QCD sum rule approach. The amount of modeling required for instanton estimates is relatively unpalatable when compared to that for the standard QCD-SRs themselves.

The QCD-SR typically regarded as the most reliable or stable nucleon sum rule has been found to be invalid. A summary of additional problems with this sum rule includes:

- The leading order $\alpha_{s}$ corrections are the order of 50\%. As such, perturbative corrections to this sum rule are very likely out of control.

- Summation of the perturbative series may give rise to an important dimension-two contribution [25,21]. The size of such a correction is presently unknown.

- Contributions from the four-quark condensate are not well known and appear to be large. Unlike the $\rho$-meson sum rule, the four-quark condensate is not suppressed by a factor of $\alpha_{s}$.

- A regime in which the OPE is reasonably convergent while the phenomenological description is dominated by the ground state pole is non-existent. If one proceeds with this sum rule, the results are very sensitive to the model for the continuum, or inaccuracies in the OPE lead to incorrect results as seen here.

Most QCD Sum Rule predictions of nucleon properties are based on this sum rule. The results are suspect, and should be reevaluated using the new techniques introduced here.

The "Ioffe formula" directly relating the nucleon mass to the local chiral condensate has been found to be misleading at best. While there is a strong connection between chiral symmetry breaking and hadron properties, such detailed or direct relationships are impossible to make and it is inappropriate to be more specific on the origin of the nucleon mass. 
The fundamental and derivative sum rules for the $\rho$ meson have also been examined. Once again, the popular sum rule analysis has been found to be undesirable. The use of derivative sum rules for the determination of $\rho$-meson spectral properties is unfavorable. Increased continuum model contributions, poorer OPE convergence, and loss of vital information in the derivative sum rule results in discrepancies between the fundamental and derivative sum rules.

The associations among the phenomenological fit parameters are particularly interesting as they reveal how the sum rules resolve the spectral properties. We have found that the separation of the ground state from the excited state continuum model is completely determined by the high dimension operators of the OPE which are not used in formulating the continuum model. The practice of fixing the continuum threshold to a preferred value has been exposed as strongly determining the outcome of the sum rule analysis. QCD sum rule determinations of phenomenological spectral properties are necessarily dependent on the treatment of high dimension operators where a factorization assumption is usually invoked. Moreover, careful attention to OPE convergence is crucial to extracting meaningful results. These issues are somewhat unsettling, as we are working with expansions which are asymptotic.

The emphasis here has been on exploring the QCD parameter space via Monte Carlo. Further refinements of the parameter space might be made by adjusting the valid Borel regime for each QCD parameter set such that the $10 \%-50 \%$ criteria are satisfied. This was not done here in order to explore other criteria and reduce the sensitivity to a reasonable yet somewhat arbitrary criteria. In addition, the Hölder inequalities might be applied as a further check of the validity and consistency of the sum rules [74].

We are now in an excellent position to attack phenomena which is of current interest to the nuclear physics community. The techniques are currently being applied to an inmedium study of vector meson properties [6]. There the debates over the behavior of the vector-meson masses and the sum rules to be used in extracting vector meson properties in nuclear matter are resolved. Using standard in-medium condensate estimates, the analysis leaves no doubt that vector-meson masses decrease with increasing density.

These techniques are also being applied to in-medium properties of the nucleon [8], by generalizing the vacuum sum rules analyzed here to finite nuclear matter density. The Monte-Carlo techniques are resolving the predictive ability of the in-medium sum rules.

Another investigation utilizing these Monte-Carlo techniques studies the QCD-SR determination of the $\rho-\omega$ meson mixing parameter $\lambda[$ [7]. There, correlations between the ratio of $u$ and $d$ quark masses are particularly interesting.

The relatively large uncertainty in the residue of the pole does not bode well for QCD Sum Rule analysis beyond two-point functions. For example, the residue of the pole appears as a pre-factor to any nucleon matrix element such as $g_{A}$ or magnetic form factors [75]. It will not be surprising to find uncertainties in these observables as large as the $35 \%$ relative

\footnotetext{
${ }^{8}$ We note that the shaded regions satisfying the Hölder inequalities in Ref. [74] are determined using both fundamental and derivative sum rules. The regions are altered when determined by the fundamental sum rule alone.
} 
error in the squared residue itself. As the uncertainties in the additional vacuum matrix elements relevant to the three-point functions are folded in the uncertainties are likely to exceed $50 \%$ and may in fact approach $100 \%$. Analyses of these issues are currently underway 76, 77.

When compared to uncertainty estimates previously published in the literature, our uncertainty estimates are somewhat larger. This is not due to any shortcoming of the approach presented here. Instead, previous authors have failed to provide a reliable method for determining realistic uncertainties. Often, uncertainty estimates have been conjectured without any supporting quantitative analysis.

This in-depth examination of QCD sum rule self consistency paints a favorable picture for further quantitative refinements of the approach. Of course such progress will be very difficult, as there are many aspects of the approach which must be refined for any particular improvement to be relevant. The techniques introduced here, such as maintaining independence of ground state properties from the interpolating field mixing parameter, might be used to further narrow the uncertainties of the approach. It is extremely important to refine the QCD-SR approach such that uncertainties in the QCD input parameters may be reduced while maintaining $\chi^{2} / N_{\mathrm{DF}} \simeq 1$. Research in this direction is required before meaningful QCD-SR-based predictions for nucleon matrix elements and moments of structure functions are possible.

\section{ACKNOWLEDGMENTS}

I am indebted to Kai Hencken for his careful checking of the Wilson coefficients and his discovery of new corrections in the dimension seven operators. His contribution is key to

resolving the discrepancy between the sum rules presented here and modern estimates of the vacuum condensates. Thanks also to Xuemin Jin for numerous beneficial discussions. Dick Furnstahl and Xuemin Jin have provided a critical examination of this analysis and I thank them for their insightful comments. Thanks also to Javed Iqbal for his contributions to the evaluation of the finite width integrals for the $\rho$-meson correlator. Many years ago, Jimmy Law provided the optimization routine used in this investigation, and I thank him for providing this useful program. This research was supported by the Natural Sciences and Engineering Research Council of Canada and the U.S. Department of Energy under grant DE-FG06-88ER40427. 


\section{REFERENCES}

[1] M. A. Shifman, A. I. Vainshtein, and Z. I. Zakharov, Nucl. Phys. B147 (1979) 385, 448.

[2] SLAC SPIRES: High Energy Physics Preprint Database, http://www-spires.slac.stanford.edu/find/top40all.htm.

[3] D. B. Leinweber, Phys. Rev. D 51 (1995) 6369.

[4] D. B. Leinweber, in Lattice '93, Proceedings of the International Symposium, edited by T. Draper, S. Gottlieb, A. Soni, and D. Toussaint, 34, (1994) 407, Dallas, TX, 1993, Nucl. Phys. B (Proc. Suppl. ).

[5] J. B. Kogut, M. P. Lombardo, and D. K. Sinclair, Phys. Rev. D 51 (1995) 1282.

[6] X. Jin and D. B. Leinweber, Phys. Rev. C 52 (1995) 3344.

[7] M. J. Iqbal, X. Jin, and D. B. Leinweber, nucl-th/9507026, Phys. Lett. B386 (1996) 55.

[8] R. J. Furnstahl, X. Jin, and D. B. Leinweber, nucl-th/9511007, Phys. Lett. B387 (1996) 253.

[9] D. B. Leinweber, Ann. Phys. (N.Y.) 198 (1990) 203.

[10] Y. Chung, H. G. Dosch, M. Kremer, and D. Schall, Z. Phys. C 25 (1984) 151.

[11] T. D. Cohen, R. J. Furnstahl, D. K. Griegel, and X. Jin, Prog. Part. Nucl. Phys. 35 (1995) 221.

[12] B. L. Ioffe, Nucl. Phys. B188 (1981) 317.

[13] D. B. Leinweber, Phys. Rev. D 51 (1995) 6383.

[14] Y. Chung, H. G. Dosch, M. Kremer, and D. Schall, Nucl. Phys. B197 (1982) 55.

[15] B. L. Ioffe, Z. Phys. C 18 (1983) 67.

[16] F. J. Dyson, Phys. Rev. 85 (1952) 861.

[17] G. 't Hooft, in The whys of subnuclear physics, edited by A. Zichichi, New York, 1977, Erice 1977, Plenum.

[18] F. David, Nucl. Phys. B234 (1984) 237.

[19] A. H. Mueller, Nucl. Phys. 250 (1985) 327.

[20] F. David, Nucl. Phys. B263 (1986) 637.

[21] V. I. Zakharov, Nucl. Phys. B385 (1992) 452.

[22] I. Bigi, M. Shifman, N. Uraltsev, and A. Vainshtein, Phys. Rev. D 50 (1994) 2234.

[23] V. A. Novikov, M. A. Shifman, A. I. Vainshtein, and V. I. Zakharov, Nucl. Phys. B249 (1985) 445.

[24] L. S. Brown and L. G. Yaffe, Phys. Rev. D 45 (1992) 398.

[25] L. S. Brown, L. G. Yaffe, and C. Zhai, Phys. Rev. D 46 (1992) 4712.

[26] D. B. Leinweber, In preparation, 1996.

[27] V. M. Belyaev and B. L. Ioffe, Sov. Phys. JETP 57 (1983) 716.

[28] B. L. Ioffe, Acta Phys. Polon. B 16 (1985) 543.

[29] S. H. Lee, S. Choe, T. D. Cohen, and D. K. Griegel, Phys. Lett. B348 (1995) 263.

[30] C. B. Chiu, J. Pasupathy, and S. J. Wilson, Phys. Rev. D 32 (1985) 1786.

[31] C. B. Chiu, J. Pasupathy, and S. J. Wilson, Phys. Rev. D 33 (1986) 1961.

[32] C. B. Chiu, J. Pasupathy, and S. J. Wilson, Phys. Rev. D 36 (1987) 1553.

[33] H. Leutwyler, Preprint CERN-TH/96-25, hep-ph/9602255, 1996.

[34] S. Narison, Phys. Lett. B361 (1995) 121, and references therein. 
[35] R. A. Bertlmann et al., Z. Phys. C 39 (1988) 231, and references therein.

[36] J. Marrow, J. Parker, and G. Shaw, Z. Phys. C 37 (1987) 103.

[37] A. D. Buskulic et al., Phys. Lett. B307 (1993) 209.

[38] V. Gimenez, J. Bordes, and J. Penarrocha, Nucl. Phys. B357 (1991) 3.

[39] S. Narison, Phys. Lett. B210 (1988) 238.

[40] V. A. Novikov, M. A. Shifman, A. I. Vainshtein, M. B. Voloshin, and V. I. Zakharov, Nucl. Phys. B237 (1984) 525.

[41] G. Launer, S. Narison, and R. Tarrach, Z. Phys. C 40 (1984) 63.

[42] V. Gimenez, J. Bordes, and J. Penarrocha, Phys. Lett. B223 (1989) 251.

[43] Particle Data Group, L. Montanet, et al. , Phys. Rev. D 50 (1994) 1729.

[44] C. Michael and A. McKerrell, Phys. Rev. D 51 (1995) 3745.

[45] C. Michael, Phys. Rev. D 49 (1994) 2616.

[46] M. J. D. Powell, Comp. J. 7 (1964) 155.

[47] W. I. Zangwill, Comp. J. 10 (1967) 293.

[48] R. P. Brent, Algorithms for Minimization without Derivatives, Prentice-Hall Inc., Englewood Cliffs, NJ, 1973.

[49] W. H. Press et al., Numerical Recipes, Cambridge University Press, New York, NY, 1986.

[50] M. J. Iqbal, X. Jin, and D. B. Leinweber, Phys. Lett. B367 (1996) 45.

[51] Y. Koike, Phys. Rev. C 51 (1995) 1488.

[52] T. Hatsuda and S. H. Lee, Phys. Rev. C 46 (1992) 34.

[53] K.-C. Yang, W. Hwang, E. Henley, and L. S. Kisslinger, Phys. Rev. D 47 (1993) 3001.

[54] H. Shiomi and T. Hatsuda, Preprint Tsukuba UTHEP-299, hep-ph/9504354, 1995.

[55] V. A. Novikov et al., Nucl. Phys. B191 (1981) 301.

[56] R. J. Furnstahl, D. K. Griegel, and T. D. Cohen, Phys. Rev. C 46 (1992) 1507.

[57] X. Jin, M. Nielsen, T. Cohen, R. Furnstahl, and D. Griegel, Phys. Rev. C 49 (1994) 464.

[58] H. G. Dosch, M. Jamin, and S. Narison, Phys. Lett. B220 (1989) 251.

[59] M. A. Shifman, Vacuum Structure and QCD Sum Rules, North-Holland, New York, 1992.

[60] H. Forkel and M. K. Banerjee, Phys. Rev. Lett. 71 (1993) 484.

[61] M. Gavela et al., Nucl. Phys. B312 (1989) 269.

[62] M. Chu, J. Grandy, S. Huang, and J. Negele, Phys. Rev. D 48 (1993) 3340.

[63] T. Schäfer, E. Shuryak, and J. Verbaarschot, Nucl. Phys. B412 (1994) 143.

[64] S. Brodsky, J. Ellis, J. Hagelin, and C. Sachrajda, Nucl. Phys. B238 (1984) 561.

[65] A. Thomas and B. McKellar, Nucl. Phys. B227 (1983) 206.

[66] Y. Tomozawa, Phys. Rev. Lett. 46 (1981) 463.

[67] M. Milosevic, D. Tadic, and J. Trampetic, Nucl. Phys. B207 (1982) 461.

[68] J. F. Donoghue and E. Golowich, Phys. Rev. D 26 (1982) 3092.

[69] N. V. Krasnikov, A. A. Pivovarov, and N. N. Tavkhelidze, Z. Phys. C 19 (1983) 301.

[70] A. Dorokhov and N. Kochelev, Z. Phys. C 46 (1990) 281.

[71] E. V. Shuryak, The QCD Vacuum, Hadrons and the Superdense Matter, World Scientific, Singapore, 1988.

[72] M. Chu, J. Grandy, S. Huang, and J. Negele, Phys. Rev. Lett. 70 (1993) 255.

[73] D. B. Leinweber, The Truth About Nucleon Sum Rules. In preparation, 1995. 
[74] M. Benmerrouche, G. Orlandini, and T. Steele, Phys. Lett. B356 (1995) 573.

[75] M. Burkardt, D. B. Leinweber, and X. Jin, hep-ph/9604450, Phys. Lett. B385 (1996) 52.

[76] F. X. Lee, D. B. Leinweber, and X. Jin, New QCD Sum Rules for Nucleon Axial Vector Coupling Constants, in PANIC '96, Proceedings of the XIV International Conference on Particles and Nucleii, River Edge, NJ, (1996), TJNAF, World Scientific Publishing Co., U. Washington PP DOE/ER/40427-10-N96.

[77] K. Hencken, E. Henley, and D. B. Leinweber, New QCD Sum Rules for Baryon Magnetic Moments. In preparation, 1996. 